\title{
Time in teams: methodological issues in the study of temporal dynamics
}

Citation for published version (APA):

Li, J. (2011). Time in teams: methodological issues in the study of temporal dynamics. [Doctoral Thesis, Maastricht University]. Time in teams: methodological issues in the study of temporal dynamics. https://doi.org/10.26481/dis.20110701jl

Document status and date:

Published: 01/01/2011

DOI:

10.26481/dis.20110701jl

Document Version:

Publisher's PDF, also known as Version of record

\section{Please check the document version of this publication:}

- A submitted manuscript is the version of the article upon submission and before peer-review. There can be important differences between the submitted version and the official published version of record.

People interested in the research are advised to contact the author for the final version of the publication, or visit the DOI to the publisher's website.

- The final author version and the galley proof are versions of the publication after peer review.

- The final published version features the final layout of the paper including the volume, issue and page numbers.

Link to publication

\footnotetext{
General rights rights.

- You may freely distribute the URL identifying the publication in the public portal. please follow below link for the End User Agreement:

www.umlib.nl/taverne-license

Take down policy

If you believe that this document breaches copyright please contact us at:

repository@maastrichtuniversity.nl

providing details and we will investigate your claim.
}

Copyright and moral rights for the publications made accessible in the public portal are retained by the authors and/or other copyright owners and it is a condition of accessing publications that users recognise and abide by the legal requirements associated with these

- Users may download and print one copy of any publication from the public portal for the purpose of private study or research.

- You may not further distribute the material or use it for any profit-making activity or commercial gain

If the publication is distributed under the terms of Article $25 \mathrm{fa}$ of the Dutch Copyright Act, indicated by the "Taverne" license above, 


\section{Time in Teams:}

Methodological Issues in the Study of Temporal Dynamics 
C Jia Li, 2011-06-07

All rights reserved. No part of this publication may be reproduced, stored in a retrieval system, or transmitted in any form, or by any means, electric, mechanical, photocopying, recording or otherwise, without the prior permission in writing from the author.

ISBN 978-94-6159-070-1

Printed by: Datawyse / Universitaire Pers Maastricht 


\title{
Time in Teams:
}

\section{Methodological Issues in the Study of Temporal Dynamics}

\author{
PROEFSCHRIFT \\ ter verkrijging van de graad van doctor \\ aan de Universiteit Maastricht, op gezag van de Rector Magnificus, \\ Prof. mr. G.P.M.F.Mols \\ volgens het besluit van het College van Decanen, \\ In het openbaar te verdedigen \\ op vrijdag 1 juli 2011 om 10.00 uur
}

door

Jia Li

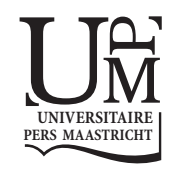




\section{Promotor}

Prof. dr. Robert A. Roe

\section{Beoordelingscommissie}

Prof. dr. Martin Carree (voorzitter)

Prof. dr. Hetty van Emmerik

Dr. Ad van Iterson

Prof. dr. Franziska Tschan (Universitê de Neuchâtel, Switzerland) 


\section{Acknowledgement}

Pursuing a doctoral degree is not an easy journey, especially for those who are far away from their homeland. As for me, I am lucky enough to meet so many wonderful people whose love and support has made the journey not only enjoyable but also meaningful.

First of all, I am very grateful to have Robert A. Roe as my promoter and supervisor. It is intellectually challenging and fun to work with Robert. From him, I have learned that good scientific work needs to be consistent in reasoning, rigorous in methods, discretionary in data, and clear in writing. He is also a role model to me in many ways with his wisdom, kindness, diligence, humbleness, patience, and pragmatic approach.

Second, I deeply thank from the bottom of my heart all the friends and colleagues inside and outside the School of Business and Economics, who have been incredibly supportive to me through the years-Ad, A-Mei, Anant, Andrea, Ann, Anneloes, Antonio, Bart D, Bert, Bram, Caren, Charlotte, Daniela, Deborah, Gabriele, GJ, Guillaume, Guy, Hannes, Hans, Hetty, Itzel, Jan, Johannes, Katrin, Maarten C, Maarten S, Martin C, Mary, Michael, Myrthe, Omar, the Raabs, Sebastian, Sjir. Thank you all very much.

Last but not least, I could never come this far without the unconditional love my parents Jianxin Li and Bin Bao gave me. They are the best parents one could possibly have. They are the real heroes. I would also like to thank my extended families and friends all over the globe, whose encouragement helped me to go through my lowest moments.

As they say, the end of one journey is the beginning of another. I will carry all what I have learned here in Maastricht and grow into a good scientist who can make you all proud. 


\section{CONTENT}

Chapter 1 Introduction 9

Two Critical Temporal Issues in Team Research 11

Stability of Team Psychological Inputs over Time 11

Team Differences in Process Dynamics 13

Aim and Scope of the Dissertation 16

$\begin{array}{ll}\text { Structure of the Dissertation } & 19\end{array}$

Chapter 2 Intertemporal Stability of Temporal Characteristics 23

Theoretical Background 26

The Intertemporal Stability of Temporal Characteristics 26

Methods $\quad 30$

Design, Procedure, and Sample $\quad 30$

Measures $\quad 30$

Analyses $\quad 32$

Results $\quad 34$

$\begin{array}{ll}\text { Discussion } & 40\end{array}$

Theoretical Implications $\quad 42$

Managerial Implications $\quad 43$

Limitations $\quad 44$

Chapter 3 Intersituational Stability of Temporal Characteristics 45

Theoretical Background $\quad 46$

$\begin{array}{ll}\text { Methods } & 47\end{array}$

Design $\quad 48$

Simulation 48

$\begin{array}{ll}\text { Procedure } & 49\end{array}$

Sample $\quad 50$

Measures $\quad 50$

Analyses $\quad 51$

Results $\quad 52$

Discussion $\quad 62$

Theoretical Implications $\quad 63$

Managerial Implications $\quad 64$

Limitations $\quad 64$

Chapter 4 An Alternative Approach to Study Team Process Dynamics 67

Two Approaches to Analyze Temporal Dynamics 70

$\begin{array}{ll}\text { Inter-subject Longitudinal Approach } & 70\end{array}$

Intra-Subject Longitudinal Approach $\quad 71$

$\begin{array}{ll}\text { Intra-Subject Longitudinal Analysis } & 72\end{array}$ 
Define Temporal Dynamics

Step 1: Depict Temporal Dynamics $\quad 82$

Step 2: Cluster Individual Subjects by Temporal Dynamics 82

Conclusion $\quad 83$

Chapter 5 The Impact of Team Conflict over Time on Team

Performance and Team Satisfaction: The Intra-Subject versus Inter-subject

$\begin{array}{ll}\text { Longitudinal Approach } & 85\end{array}$

$\begin{array}{ll}\text { Theoretical Background } & 88\end{array}$

Punctuated Equilibrium Model and Temporal Dynamics of Team Processes $\quad 88$

Task Conflict over Time $\quad 89$

Relationship Conflict over Time $\quad 92$

Process Conflict over Time 93

$\begin{array}{ll}\text { Methods } & 96\end{array}$

Research Setting, Procedure, and Sample 96

$\begin{array}{ll}\text { Measures } & 97\end{array}$

Analyses $\quad 98$

$\begin{array}{lr}\text { Results } & 99\end{array}$

$\begin{array}{ll}\text { Discussion } & 110\end{array}$

$\begin{array}{ll}\text { Theoretical Implications } & 110\end{array}$

Managerial Implications 114

$\begin{array}{ll}\text { Limitations } & 114\end{array}$

$\begin{array}{ll}\text { Chapter } 6 \text { Discussion and Conclusions } & 117\end{array}$

$\begin{array}{ll}\text { Review of Our Findings } & 119\end{array}$

Implications for Future Research $\quad 121$

Using Team Inputs as Predictors $\quad 114$

Examining Heterogeneous Temporal Dynamic Patterns of a Team Process 121

Analyzing the Role of Team Processes 122

Other Implications $\quad 125$

Managerial Implications $\quad 127$

$\begin{array}{ll}\text { Limitations } & 127\end{array}$

$\begin{array}{lr}\text { References } & 129\end{array}$

$\begin{array}{ll}\text { Appendix A } & 137\end{array}$

$\begin{array}{ll}\text { Appendix B } & 142\end{array}$

$\begin{array}{ll}\text { Appendix C } & 162\end{array}$

$\begin{array}{ll}\text { Summary } & 171\end{array}$

$\begin{array}{ll}\text { Samenvatting (Summary in Dutch) } & 175\end{array}$

$\begin{array}{lr}\text { Curriculum Vitae } & 179\end{array}$ 



\section{Chapter 1 \\ Introduction}

Team researchers have spent considerable efforts in understanding the effectiveness of work teams using an Input-Process-Output (IPO) framework. According to the framework, team inputs (e.g. team composition in demographics and personality traits) account for team outputs (e.g. performance, satisfaction) through team members' interactions (e.g. coordination, conflict management). Although researchers have tried to improve the models by including "deep-level" team psychological inputs and conceptualizing team processes as dynamic over time, empirical results have remained modest and inconsistent.

The research reported in this dissertation aims to advance team research by taking a temporal-dynamic perspective in examining the causal links among team inputs, team processes, and team outputs and by addressing some critical methodological issues related to time. First, it examines whether team psychological inputs are sufficiently stable over time and across situations to display meaningful relations with team processes and team outputs. $\mathrm{We}^{1}$ investigate the intertemporal and intersituational stability of four temporal characteristics, that is, time urgency, polychronicity, time perspective, and temporal depth. Second, it scrutinizes the research methods used in extant team process research and points out that the commonly used methods may have failed to address the inter-team differences in intra-team process dynamics over time and provide limited insights in the relationships between temporally dynamic team processes and team outputs. We develop a new research approach - designated as the 'intra-subject longitudinal approach'- to study team process dynamics over time. We also apply the developed approach in an empirical team study and compare the findings with those from the conventional inter-subject longitudinal approach used in most team process research.

\footnotetext{
${ }^{1}$ In Chapter 1, 3, 4, 5, and 6, "we" refers to Jia Li and Robert A. Roe. In Chapter 2, "we" refers to Jia Li, Robert A. Roe, and Mary J. Waller.
} 
Team researchers have often used an Input-Process-Output (IPO) framework (Cohen \& Bailey, 1997; Hackman, 1987; McGrath, 1984) to explain the effectiveness of work teams. The logic of the framework is that team outputs (e.g. performance, satisfaction) are accounted for by team inputs (e.g. skills, demographics, personality traits), contextual inputs (e.g. task characteristics, reward structures), and team processes (e.g. communication, conflict). For example, in team diversity research, early studies have examined the impact of team demographic diversity (e.g. team diversity in gender, age, and ethnicity) on team social outputs (e.g. social integration, organizational attachment, turnover, O'Reilly, Caldwell, \& Barnett, 1989; Tsui, Egan, \& O'Reilly, 1992; Wagner, Pfeffer, \& O'Reilly, 1984). More recent studies have examined how team processes, such as team internal and external communication and team conflict, help to explain the effect of team demographic diversity on team performance outputs (See the studies by Ancona and Caldwell, 1992, Jehn, Northcraft, and Neale, 1999, Pelled, 1996, and Pelled, Eisenhardt, and Xin, 1999, for examples).

Despite its wide application in team research and numerous contributions to our understanding of (un)successful teamwork, the IPO framework bears one major limitation, that is, the inconsistency of its predictions. For example, in top management team (TMT) research, some researchers (Barsade, Ward, Turner, \& Sonnenfeld, 2000) have found that the diversity of team members' functional background benefits firm performance, whereas other researchers (Simons, Pelled, \& Smith, 1999) report the opposite, detrimental effect. In order to improve the framework's explanatory and predictive power, researchers have proposed two new research directions. The first direction is to theorize the influence of team members' psychological attributes in explaining the impact of team demographic composition ${ }^{2}$ on team outputs. The argument goes that in addition to demographic features (e.g. gender, ethnicity, functional background), team members' psychological attributes (e.g. attitudes, values, personality traits) also influence teams' performance outputs and social outputs. For example, in team diversity research, researchers have argued that the law of similarity-attraction (Byrne, 1971) can be based on both demographic features and psychological attributes. Over time, team members are more likely to identify themselves with others with similar psychological attributes than with those with similar demographic features (Harrison, Price, \& Bell, 1998; Harrison, Price, Gavin, \& Florey, 2002). Team psychological inputs (i.e. the "deep-level" team composition) account for some variances in team outputs that are not explained by team demographic attributes (i.e. the "surface-level" team composition).

The second direction to improve the framework is to investigate the "black box" of team processes, that is, to understand how, or through what team interactions, team inputs affect team outputs (Lawrence, 1997). For example, in a study on new product development teams in high-tech industries, Ancona and Caldwell

\footnotetext{
${ }^{2}$ In this dissertation, team composition includes teams' mean, minimum, and maximum level and within-team distribution of a demographic feature or psychological attribute.
} 
(1992) find that teams' external communication activities explain why teams with diverse team members' functional background tend to achieve a higher level of innovation performance than teams whose members have a similar functional background. Extensive external communication can help teams to acquire various types of information and stimulates innovative ideas that are essential for high innovation performance. Team processes (i.e. what teams do) mediate (i.e. explain) and moderate (i.e. change) the impact of team inputs - deep-level or surface-level - on team outputs.

Although not explicated in the previous literature, two temporal conditions must be fulfilled in order for the two proposed research directions to make the expected contribution. The first condition is that team psychological inputs are sufficiently stable over time and across situations, so that their predictions are valid and consistent, rather than contingent on specific measurement moments. The second condition is that the temporal dynamics of a team process differs across teams, so that the inter-team differences in the intra-team ${ }^{3}$ process over time are linked to inter-team differences in team input levels and/or team output levels in a theoretically and statistically meaningful way. In the remaining chapter, we review and discuss how the two conditions are dealt with in the current organizational behavior literature and conclude that they should be addressed in a more adequate way. Next, we outline the aim, scope, and structure of the dissertation.

\section{Two Critical Temporal Issues in Team Research}

\section{Stability of Team Psychological Inputs over Time}

In real-life organizations, team inputs, including surface-level demographic features and deep-level psychological attributes, may change over time, as teams continue to function. For example, new members joining a team may change the team's gender distribution and introduce new skills and personality traits to the team. Moreover, some demographic and psychological characteristics (e.g. functional background, work values) of team members may change due to aging, gaining experience, or interactions within and outside the team. However, in team research based on the IPO framework, team inputs are often conceptualized as static over time and across situations, and - once measured - are expected to provide valid and consistent predictions of team processes and team outputs. Little attention is given to the intertemporal and intersituational stability ${ }^{4}$ of team inputs. Psychological attributes' intertemporal stability (i.e. stability over a given time interval) has been typically examined by means of the test-retest reliability coefficient. The test-retest reliability

\footnotetext{
${ }^{3}$ In this dissertation, "intra-team" refers to of one team over time and contrasts to "inter-team" or "between-teams". An intra-team process can be internally focused, such as communication among team members, or externally focused, such as communication to teams' external stakeholders.

${ }^{4}$ In this dissertation, "stability" refers to the degree of being stable rather than the state of being stable.
} 
coefficient is a correlation coefficient, in which the attribute scores of the same group of subjects are obtained twice in time and measured by the same instrument. For example, Big Five personality traits (i.e. neuroticism, extraversion, openness, agreeableness, and conscientiousness) are concluded to be stable personality traits, after Costa and McCrae (1988) examined the traits' stability in the same sample of adults over six years via the test-retest reliability coefficient and the NeuroticismExtraversion-Openness Personality Inventory (NEO-PI) as measurement. They report that, for example, being open to new experiences is a more stable personality trait than being extraverted, since the test-retest coefficient of the former is .80 , in contrast to .77 of the latter. Inter-person differences of the attribute scores were more consistent for openness than for extraversion over the six years period.

There are two methodological drawbacks in using the test-retest reliability coefficient to examine the intertemporal stability of a psychological attribute. First, it assumes that the measurement instrument of a psychological attribute is equivalent over time, and that the observed attribute scores solely reflect subjects' true attribute levels. It discounts the possibility that change in the observed attribute scores may originate from subjects' change in the understanding of the measurement items and/or from the instrument's recalibration caused by repeated measurement. Golembiewski, Billingsley, and Yeager (1976) note three types of attribute change over time, that is, change in construct validity, change in calibration, and change in attribute level, which are labeled "gamma change", "beta change", and "alpha change" respectively. The potential confounding effect of gamma change and beta change on the change (or stability) of observed attribute scores represents a particular and well-known risk in longitudinal research that employs subjective measurement.

Second, the test-retest reliability coefficient indicates change in the relative positions of a group of subjects' attribute scores over time, rather than change of the group's or each subject's attribute score over time. It indicates to what degree individuals' rankings within the group have changed over time, rather than to what degree the group's mean attribute score or individuals' attribute scores have changed. If, for example, all subjects in a sample increase their scores of an attribute to the same degree between two time moments, a high test-retest coefficient will result. This may mislead researchers to conclude the attribute is stable over time, despite the fact that the group's mean attribute score and all individuals' attribute scores have changed over time.

In order to overcome these drawbacks, a methodology is needed to examine the measurement equivalence of an attribute over time before examining the stability of the attribute score over time in a way that separates the stability of subjects' rank orders of the attribute scores. Such a methodology has been proposed by Chan (1998) and Lance, Vandenberg, and Self (2000). It tests a set of progressively restricted structural equations and distinguishes between measurement equivalence over time and attribute score stability over time, as well as between the stability of 
subjects' attribute score rankings over time and the stability of subjects' attribute scores over time ${ }^{5}$.

\section{Team Differences in Process Dynamics}

The IPO framework implies that team processes are intermediary, that is, mediate or moderate the relationship between team inputs and team outputs. Team processes are conceived as team interactions within a team and with external parties (Cohen \& Bailey, 1997; McGrath, 1984). Marks, Mathieu, and Zaccaro (2001:357) define team processes as '(team) members' interdependent acts that convert inputs to outcomes through cognitive, verbal, and behavioral activities directed toward organizing taskwork to achieve collective goals." And "different forms of team processes describe the types of interactions that take place among team members during the course of goal accomplishment (2001:357)." The essence of the concept is team interactions over a certain period of time. Team processes are inherently dynamic ${ }^{6}$ and imply developmental trajectories of team interactions over time. Accordingly, the empirical investigation of team processes should follow a temporal-dynamic approach (for elaboration, see Roe's [2008] and Solinger's [2010] studies). It should conceive a team process as a temporally integral phenomenon that reflects "what happens" in a team and relate inter-team differences in team process dynamics to inter-team differences in team input and/or output levels. However, most empirical studies of team processes follow a differential approach in which team process levels are either measured once in time and entered into regression models, or measured repeatedly over time but studied discretely, that is, team process levels are linked to team input and/or output levels on a one-by-one basis, before being charted over time.

In the extant literature, team processes are measured and examined in the five following ways. First, team tenure is used as a proxy for real-time team processes (see Harrison and colleagues' [2002] study for an example); actual team interactions are neither measured nor studied. Second, in many cases, team processes are measured once in time and studied as mediators or moderators of the relationships between team inputs and team outputs (see Jehn, Northcraft, and Neale's [1999] and Pelled, Eisenhardt, and Xin's [1999] studies for examples). Thus, an inherently dynamic team process is conceptualized as a static phenomenon and studied by an inherently static variances-based differential approach (Van de Ven, 2007). Third, in some studies, team interactions are observed (or proposed to be observed) in real time, and the developmental trajectories of teams' task progress and social interactions over time are depicted. Among all, Tuckman's (1965) five-stage team devel-

\footnotetext{
${ }^{5}$ However, the testing procedure does not separate the test between group-level and individual-level attribute score change over time. In Chapter 4, we will elaborate on the logical connection between the two levels.

${ }^{6}$ In this dissertation, "dynamic" or "dynamics" refers solely to temporal dynamics or dynamics over time.
} 
opment model and Gersick's $(1988,1991)$ punctuated equilibrium model are the most notable examples of this type of research. The five-stage model suggests that teams develop gradually over time, progressing through forming, storming, norming, performing, and adjourning stages sequentially. The punctuated equilibrium model suggests that teams evolve discretely over time, "jumping" from an inertial state to an active state at a certain moment of time during team project. In these cases, team process dynamics is observed in real time, but not related to team input and/or output levels. As Hackman (1987) notes, such research is descriptive rather than causal. Fourth, in some studies, team interactions are observed in real time, and the causal links between team process dynamics and team output levels are established via qualitative analysis. For example, Gersick (1989) finds that project teams that reorient themselves at the exact middle time point of team projects outperform those who do not. Ericksen and Dyer (2004) report that project teams' early mobilization strategies affect their final performance. Thus, causality is established qualitatively rather than through compelling statistical inference. Fifth, in some studies, team interactions are measured in real time, and the causal links between team process dynamics and team input and/or output levels are established via statistical inference. The inference in these studies is typically based upon an association between team input and/or output levels and the levels of team processes measured at different time moments, rather than between team input and/or output levels and the temporal dynamics of team processes as a gestalt variable. For example, when studying the effect of team conflict (i.e. task conflict, relationship conflict, process conflict) over time on team performance, Jehn and Mannix (2001) first examine whether high-performing teams and low-performing teams differ in the level of team conflict at the beginning, middle, and end of team projects and, consequently chart the subsamples' (i.e. high-performing teams versus lowperforming teams) mean conflict levels across the three time moments. Their findings, although highlighting the importance of time in studying team conflict, bespeak little the impact of team conflict, as an inherently dynamic and temporally integral phenomenon, on team performance.

Neither of these approaches to the study of team processes is fully satisfactory. First, team tenure as a proxy for real-time team interactions and single-moment measurement of inherently dynamic team processes collapses team processes into a static snapshot and therefore excludes rich information about what is actually happening in teams. As McGrath (1984) noted earlier, such a treatment is not adequate, mismatching a dynamic-by-nature research phenomenon (i.e. team processes) with a static-by-nature differential approach.

Second, describing team developmental stages over time (Bales, 1950; Gersick, 1988; Tuckman, 1965), although stimulating the study of team processes, contributes little to our understanding of whether and how inter-team differences in team processes leads to inter-team differences in team final outputs. As Hackman (1987: 316) criticized earlier, descriptive team process research, compared with 
causal investigations, is a research practice that "produce[s] neither a set of empirical generalizations sturdy enough to guide managerial practice nor interventions that reliably improve group performance."

Third, the conventional statistical inference from team processes over time to team output levels (see Jehn and Mannix's [2001] study for an example) displays a logical flaw. Researcher first examine the association between team output levels and the levels of a team process at successive time moments, and then chart the team process' temporal dynamics of a sample or subsample ${ }^{7}$. This approach, which we designate as inter-subject longitudinal approach, thus examines "how teams with different output levels differ in team process levels over time." It follows a temporally-backward logic and implies that teams with a similar output level must have had homogeneous developmental pattern ${ }^{8}$ of a team process over time and that group-level (e.g. sample-level, subsample-level) dynamic pattern, once known, automatically informs individual dynamic patterns. However, the theoretical inquiry in the team process-output link pertains to "whether and how teams with different process dynamics over time differ in subsequent team output levels" and follows temporally-forward logic. To examine the process-output link, researchers need an approach that matches the purpose of the theoretical inquiry. The alternative approach should acknowledge that team processes precede team outputs in time and that teams may differ their dynamic patterns of a team process over time. A few notable real-time empirical observations have actually shown heterogeneous developmental trajectories of project teams' task progress and social interactions over time (Chang, Bordia, \& Duck, 2003; Ericksen \& Dyer, 2004; Gersick, 1989; Okhuysen \& Waller, 2002). Accordingly, the alternative research approach requires a different conceptualization of the relationship between group-level and individuallevel temporal dynamics and another method of analysis.

In order to understand the impact of team processes - dynamic-by-nature and idiosyncratic team interactions - on team output levels, researchers need not only to measure team processes repeatedly over time but also to acknowledge the uniqueness of team interaction patterns over time. No proxy, single measurement, or measurement of group-level temporal dynamics can sufficiently reveal what actually happens in teams, how teams differ in their interaction patterns over time, what team inputs may lead to such differences, and what team outputs may result from them. As Hackman (1987: 319) has noted, "few social psychological studies have addressed the possibility that groups [or work teams] might perform better if members work together in ways that differ from typical interaction patterns. Argyris [1969] argues that this is a serious failure of social psychology theory." Hence, it is imperative for team researchers to study inter-team differences in intra-team process dynamics over time in their own right, rather than treating team process dynam-

\footnotetext{
${ }^{7}$ Subsamples are formed based on teams' output levels.

${ }^{8}$ In this dissertation, "dynamic patterns", "developmental patterns", and "developmental trajectories" share the same meaning and are therefore used interchangeably.
} 
ics as simply identical across teams or randomly deviant from the "averaged" group-level pattern. The required alternative research approach should better align with the theoretical objective and with the empirical reality than the conventional inter-subject longitudinal approach.

\section{Aim and Scope of the Dissertation}

The overarching aim of the dissertation is to contribute to team effectiveness research by examining two critical yet under-studied temporal conditions in the team IPO framework. These conditions are (1) that team psychological inputs are sufficiently stable over time and across situations and (2) that the temporal dynamics of a team process differ across teams, so that inter-team differences in intra-team process dynamics are associated with inter-team differences in team input and/or output levels. We apply an advanced conceptualization and testing method to examine group-level attribute changes over time, reconceptualize the relationship between group-level and individual-level temporal dynamics, develop a new intra-subject longitudinal approach to examine individual-level temporal dynamics, and apply both the intra-subject longitudinal approach and the conventional inter-subject longitudinal approach in an empirical study of the impact of team conflict over time on team performance and team satisfaction.

To examine the first condition, we test the intertemporal and intersituational stability of four individual temporal characteristics, namely, time urgency, polychronicity, time perspective, and temporal depth. Time urgency is an individual tendency to "consider time as a scarce resource and plan its use carefully (Landy, Rastegary, Thayer, \& Colvin, 1991:645)." Polychronicity is a preference to perform multiple activities at the same time (Hall, 1983; Slocombe \& Bluedorn, 1999). Time perspective is "a non-conscious process whereby the continual flows of personal and social experiences are assigned to temporal categories, or time frames, that help to give order, coherence, and meaning to those events (Zimbardo \& Boyd, 1999:1271)." Temporal depth is "the temporal distance into the past and the future that individuals and collectivities typically consider when contemplating events that have happened, may have happened, or may happen (Bluedorn, 2002: 114)."

We include these four temporal characteristics, because they capture four important aspects of the human experience of time and are theorized and found to be valid predictors of time-related team processes (e.g. attention to time) and team performance (e.g. productivity-by-time, meeting project deadlines, Waller, Conte, Gibson, \& Carpenter, 2001; Waller, Giambatista, \& Zellmer-Bruhn, 1999). The intertemporal stability of time urgency, polychronicity, and time perspective has been examined via the test-retest reliability coefficient (Bluedorn, Kalliah, Strube, \& Martin, 1998; Landy et al., 1991; Zimbardo \& Boyd, 1999). No empirical evidence has been provided about the intertemporal stability of temporal depth. The 
aforementioned methodological drawbacks of the test-retest reliability coefficient prompt us to employ an advanced conceptualization and testing method to (re)assess the intertemporal and intersituational stability of the four temporal characteristics.

The alternative methodology comprises a repeated measurement design with three time moments, a distinction between measurement instrument stability and attribute score stability over time, and a latent growth modeling procedure with structural equations. It is based on Golembiewski and colleagues' (1976) alphabeta-and-gamma-change typology and Chan (1998) and Lance and colleagues' (2000) testing procedure of attitude change over time. It does not only decompose change in observed subjects' attribute scores into construct validity change, calibration change, and construct score change, but also distinguishes between change in subjects' score rankings and change of the group's mean score over time.

To examine the second condition, we first develop an intra-subject longitudinal approach and then apply both the intra-subject and inter-subject longitudinal approach in an empirical study on the impact of team conflict over time on team performance and team satisfaction. Conflict is "an awareness on the part of the parties involved of discrepancies, incompatible wishes, or irreconcilable desires" (Boulding, 1963, quoted in Jehn \& Mannix, 2001: 238). Team conflict is known to appear in three forms, that is, task conflict, relationship conflict, and process conflict (Jehn, 1995, 1997; Jehn \& Mannix, 2001). Task conflict is "an awareness of differences in viewpoints and opinions pertaining to a group task" (Jehn \& Mannix, 2001: 238). Relationship conflict is "an awareness of interpersonal incompatibilities, including affective components such as feeling tension and friction" (Jehn \& Mannix, 2001: 238). Process conflict is "an awareness of controversies about aspects of how task accomplishment will proceed, [which] pertains to issues of duty and resource delegation, such as who should do what and how much responsibility different people should get" (Jehn \& Mannix, 2001: 239).

We focus on team conflict, because it has been studied extensively in the team literature and was found to be one of the most crucial team processes that affect team performance and team social outputs (for a review, see two meta-analysis studies by De Dreu and Weingart [2003], and De Wit and Greer [2008]). The overall empirical evidence suggests that team task, relationship, and process conflict are negatively related to team performance and team social outputs (i.e. consequences), but positively related to team demographic diversity (i.e. antecedents). Moreover, team conflict has been found to mediate the negative effect of team demographic diversity on team performance. Using the inter-subject approach, Jehn and Mannix (2001) report that high-performing teams and low-performing teams differ in their conflict levels over time and that high performance is associated with moderate levels of task conflict at the middle time point of team projects, low levels of relationship conflict with a rise near project deadlines, and low but increasing levels of process conflict over time. However, the aforementioned incompatibility between 
the temporally-backward logic of the inter-subject approach and the temporallyforward logic of the theoretical inquiry prompts us to develop an alternative intrasubject longitudinal approach that aligns with the theoretical inquiry and empirical reality.

The intra-subject approach is characterized by a repeated measurement design, a temporally-forward logic, an assumption of heterogeneous intra-subject process dynamic patterns, and an individuals-to-group analysis method. Although the approach can be applied to longitudinal research on intra-person and intra-team attitudinal and behavioral change, we illustrate it with team research that applies the IPO framework. First, team processes need to be measured three (or more) times. Punctuated equilibrium theory (Gersick, 1988, 1989, 1991), a primary team development theory developed from real-time observations of project teams' interactions, suggests that the beginning, middle, and end of team projects are three critical time moments during project team functioning. Second, the temporally-forward logic acknowledges that team processes precede team performance and team social outputs in time, and suggests that team outputs can be inferred from empirically observed team processes. It differs from the temporally-backward logic in the intersubject approach, which infers intra-team process dynamics from the associations between team process levels over time and team output levels, in other words, "by specifying what [team] members may have done or logically had to have done to account for the results (Hackman, 1987: 318)." Third, the heterogeneity assumption implies that teams may differ in their internal and external interactions over time, which fits the observed empirical reality better than the homogeneity assumption of the inter-subject approach. Fourth, the individuals-to-group analysis method, derived from the heterogeneity assumption, constructs group-level dynamic pattern from the distribution of heterogeneous individual dynamic patterns, whereas the group-to-individual method of the inter-subject approach, derived from the homogeneity assumption, decomposes the "averaged" group pattern into homogenous individual patterns. In the new intra-subject approach, the research focus is, rather than the level of a team process across time moments, the temporal dynamics of the team process as a whole.

The intra-subject longitudinal approach contributes to the team research in the IPO-framework in several ways. It acknowledges inter-team differences in intrateam process dynamics over time, connects group-level and individual-level temporal dynamics through observed heterogeneous individual patterns (rather than presumed homogeneous individual patterns), establishes the causal links among team inputs, team processes, and team outputs via statistical inference, and, most importantly, examines the temporally-forward research questions (i.e. team processes precede and predict team outputs) with a research method that follows a compatible temporally-forward logic. 


\section{Structure of the Dissertation}

This dissertation consists of six chapters. In Chapter 2, we test the intertemporal stability of time urgency, polychronicity, time perspective, and temporal depth in a sample of 63 faculty members and students of a Dutch university over a period of six months. In a naturalistic scenario, we administer questionnaires measuring these attributes three times in three consecutive academic quarters. We analyze the data via the alpha-beta-gamma-change typology and a procedure of latent growth modeling (LGM).

In Chapter 3, we test the intersituational stability of time urgency, polychronicity, time perspective, and temporal depth in a quasi-experiment with two groups of business students. We test both groups twice over a period of one month. In the control group $(n=49)$, we measure the temporal characteristics twice in the same naturalistic "weak" situation. In the experimental group $(n=52)$, we measure the temporal characteristics first in a naturalistic "weak" situation and later in a simulated "strong" situation, designed to affect participants' time perceptions. We analyze the data via the alpha-beta-gamma-change typology and LGM procedure, and compare the results between the groups. We assess the measurement instrument equivalence and attribute score stability of the four temporal characteristics between the "weak-weak" and "weak-strong" conditions.

In Chapter 4, we discuss the relationship between group-level and individuallevel temporal dynamics and two ways to study it, that is, inferring individual-level dynamics from group-level dynamics (i.e. the group-to-individuals decomposition method) and inferring group-level dynamics from individual-level dynamics (i.e. the individuals-to-group aggregation method). Next, based on the discussion, we develop an intra-subject longitudinal approach to study intra-subject change over time and its relations to theorized antecedents and consequences. The new intrasubject approach conceives individual subjects' temporal dynamics of a psychological attribute as a gestalt phenomena and variable, acknowledges heterogeneous individual dynamic patterns, constructs group-level dynamic pattern from the distribution of heterogeneous individual patterns. We further illustrate the approach with team process research with three measurement moments and shortly discuss its merits and limitations.

In Chapter 5, we examine the impact of team conflict over time on team performance and team cohesion via the intra-subject and the inter-subject longitudinal approach. We use data from 42 project teams (121 persons) consisting of business graduate students who are required to develop research proposals during a twomonth course. We measure team task, relationship, and process conflict at the beginning, middle, and end of the course and analyze the impact of the level and dynamics of team conflict over time on team performance and team satisfaction. We present and compare the results of the two approaches. 
In Chapter 6, we review and discuss the findings of the dissertation. We emphasize the necessity for team researchers to test the intertemporal and intersituational stability of a psychological attribute with the alpha-beta-gamma-change typology and the latent growth modeling procedure before using it to predict team processes and team outputs. When a temporal characteristic shows low intertemporal and/or intersituational stability at the group level, it should not be used to predict time-related team processes (e.g. pacing behavior, attention to time) or time-related team performance (e.g. productivity-by-time, meeting project deadlines), because such research practice may lead to erroneous conclusions. We argue that the intrasubject longitudinal approach is a feasible and effective methodology for investigating dynamic and heterogeneous team processes and their intermediary (i.e. mediating and moderating) effects on the relationship between team inputs and team outputs. Finally, we discuss some practical implications of our research as well as the limitations of our work. We summarize the theoretical inquiries, research questions and research methods of the five following chapters in Table 1.1. 


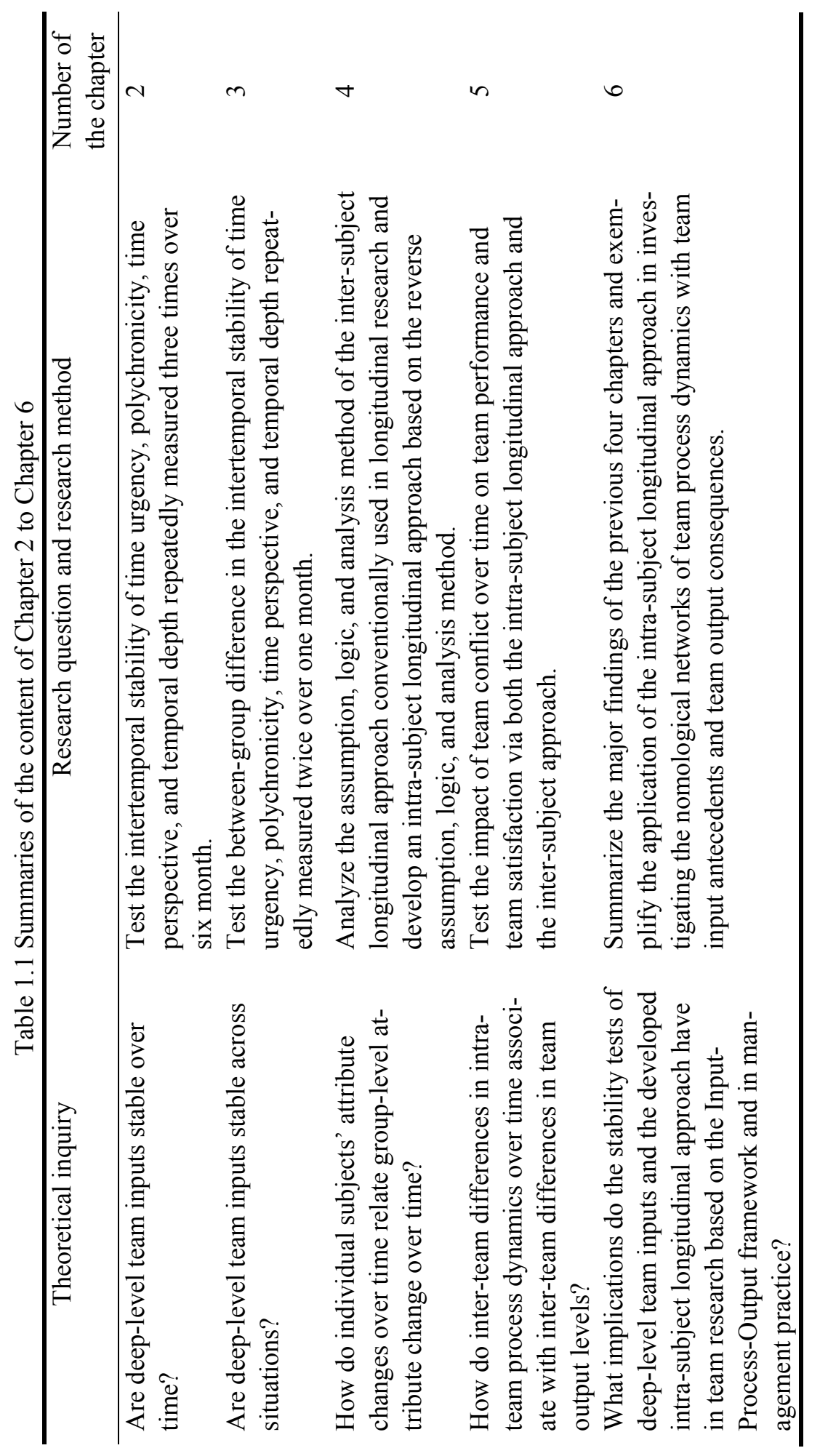





\section{Chapter 2 \\ Intertemporal Stability of Temporal Characteristics ${ }^{9}$}

Time plays two important roles in team research based on the Input-Process-Output (IPO) framework. The first role is a methodological one, in which it underlies the two critical conditions mentioned in Chapter 1, that is, deep-level team inputs must be stable over time and team processes as a temporally integral phenomenon must differ across teams. The second role is substantive and pertains to team members' experience of time and its impact on the progress of team tasks and team performance over time. Research on the subjective time is particularly important in understanding the effectiveness of project teams, a major carrier of organizations' innovation tasks (Hülsheger, Anderson, \& Salgado, 2009), because project teams' functioning is by nature time-bounded (Cohen \& Bailey, 1997) and their performance is partially measured by meeting project deadlines and speed of delivery (Brown \& Eisenhardt, 1995). It is theorized in recent team research that team members' time urgent feelings and orientation towards past, present, or future may influence project teams' perception of deadlines during the team process and hence their chances to successfully meet the deadlines (Waller, Conte, Gibson, \& Carpenter, 2001). Given the importance of stable "deep-level" team inputs in predicting team processes and team outputs, it is crucial to test the intertemporal and intersituational stability of individual temporal characteristics (e.g. time urgency, time perspective) before further using them as "deep-level" team inputs to predict project teams' time-related processes and performance outcomes.

In this study, we test the stability of time urgency, polychronicity, time perspective, and temporal depth over a period of six months. Following the reasoning of Golembiewski, Billingsley, and Yeager (1976), the intertemporal stability of an attribute's measured score reflect the intertemporal stability of the attribute score if, and only if, the attribute's measurement instrument has demonstrated sufficient

\footnotetext{
${ }^{9}$ An early version of this chapter was presented at the 2008 Annual Meeting of the Academy of Management in Anaheim, California, the United States. An updated version is currently under review at a peer-reviewed journal (c.f. Li, J., Waller, M.J., \& Roe, R.A., Temporal characteristics as deep-level team inputs: An examination of their stability, Manuscript submitted for publication.)
} 
stability in validity and calibration over time (i.e. the typology of alpha, beta, and gamma change). To test the intertemporal stability of the four temporal characteristics, we apply the latent growth modeling (LGM) procedure used in Chan's (1998) and Lance, Vandenberg, and Self's (2000) studies. Our results show that the measurement of most time urgency and time perspective dimensions and of the unidimensional polychronicity varies over time, demonstrating validity or calibration change over time (i.e. gamma or beta change). In comparison, future-orientation of time perspective and temporal depth are rather stable over time. We discuss the implications of the study for future team research and management practice.

In the past two decades, a great deal of research has been dedicated to understanding people's time experience and the impact of individual temporal characteristics on work-related behaviors and performance (Ancona, Okhuysen, \& Perlow 2001; McGrath, 1986). Research shows, for example, that time urgent individuals, who feel constantly pressured by time and who work fast, are often punctual (Dishon-Berkovits \& Koslowsky, 2002) and deliver above-average job performance (Greenberg, 2002). Polychronic individuals, who prefer to perform multiple tasks simultaneously, have higher publication productivity as faculty members and better sales performance as sales representatives than monochronic individuals, who prefer to perform multiple tasks one after another (Conte \& Gintoft, 2005; Taylor, Locke, Lee, \& Gist, 1984). Team researchers have recognized the importance of temporal characteristics in team research and have suggested that they are an important set of team psychological inputs that may influence team processes and team outputs. For example, Waller and colleagues (Waller, Conte, Gibson, \& Carpenter, 2001; Waller, Giambatista, \& Zellmer-Bruhn, 1999) theorized an impact of team members' time urgency (i.e. a feeling of being pressured by time) and time perspective (i.e. a cognitive focus on the past, present, or future) on team deadline meeting performance and found a positive impact of time urgency on team performance. Mohammed and Angell (2004) found a positive impact of team time urgency diversity on team relationship conflict, mediated by team cooperation and communication.

Before temporal characteristics are employed to predict team processes and team outputs, one critical condition must be established, that is, temporal characteristics are stable over time and across situations. This is important to prevent timecontingent findings. For example, if team members' feelings of time urgency vary considerably throughout the team functioning period, the moment at which time urgency is measured in relations to teams' developmental stages or life expectancy may affect its power to predict team processes and team outputs and generate inconsistent results across studies. Therefore, in this dissertation, we examine the intertemporal stability of temporal characteristics (Chapter 2) and the intersituational stability of temporal characteristics (Chapter 3).

Traditionally, the intertemporal stability of an attribute is examined by the test-retest reliability coefficient. An attribute is measured twice with one measure- 
ment instrument and the correlation between the measurement moments is seen as indication of stability. The method has two major methodological drawbacks. First, it assumes that change in measured attribute scores reflect solely change in subjects' attribute levels and omits that change in the measured scores may result from change in the measurement instrument itself. Golembiewski, Billingsley, and Yeager (1976) proposed to decompose change in measured attribute scores into construct validity change (i.e. gamma change), construct calibration change (i.e. beta change), and attribute level change (i.e. alpha change). Later, Chan (1998) and Lance, Vandenberg, and Self (2000) argued for establishing temporally equivalent measurement, that is, equivalent construct validity and calibration over time, before examining attribute level change over time. Second, the test-retest reliability coefficient assesses only the rank order change of a group of subjects over time, but not subjects' attribute level change over time. If all subjects in a sample changed their attribute levels to the same extent over time, the test-retest reliability coefficient would indicate high intertemporal stability of the attribute, in spite of the occurrence of attribute level change. Therefore, it is important to distinguish between subjects' rank order change and their attribute level change over time.

This study aims to examine the intertemporal stability of temporal characteristics by an advanced methodology that overcomes the two methodological drawbacks of the test-retest reliability coefficient. This methodology builds on the alphabeta-gamma-change typology (Golembiewski et al., 1976) and tests of a set of structural equation models (Chan, 1998; Lance et al., 2000). In particular, we test six progressively restricted structural equation models, and one additional model, for time urgency, time perspective, and polychronicity. Since time urgency and time perspective are multidimensional, we examine each of their dimensions separately. We also test the intertemporal stability of temporal depth, using repeated-measured Analysis of Variances (ANOVA).The reason for this is that, unlike the other temporal characteristics, each dimension of temporal depth (e.g. short-term past temporal depth, short-term future temporal depth), is measured by one questionnaire item. We selected these four temporal characteristics, because they represent four important aspects of human experience of time that the recent team literature (e.g. Waller et al., 1999, 2001; Mohammed \& Angell, 2004) sees as critical deep-level team inputs that affect teams' time-related processes (e.g. pacing, attention to time) and performance (e.g. productivity-by-time, meeting project deadlines). The following chapter unfolds by a brief description of the four temporal characteristics and previous research on their intertemporal stability. Then we present a longitudinal study that examines the intertemporal stability of the four temporal characteristics. Finally, we discuss the implications of the study for future team research and management practice. 


\section{Theoretical Background}

\section{The Intertemporal Stability of Temporal Characteristics}

Despite the rising importance of team members' temporal characteristics in team research (Waller et al., 1999, 2001; Mohammed \& Angell, 2004), it remains unknown whether temporal characteristics are stable over time and across situations or may change as a result of, for example, team members' learning and adaptation to change in teams' internal and external environment. In previous research, time urgency and time perspective were typically assumed to be stable over time and across situations and therefore measured only once in time. The assumption of sufficient intertemporal stability is based on evidence of sufficiently high test-retest reliability, which, as we noted before, does not allow inferences of attribute level change over time. Evidence on intersituational stability is lacking. To our knowledge, this study and the study in Chapter 3 are the first to apply the more advanced latent growth methodology to assess the intertemporal and intersituational stability of temporal characteristics and provide evidence of the stability of temporal characteristics stability in future team research.

Time urgency. Time urgency, a key component of Type A Behavior Patten (TABP, Friedman \& Rosenman, 1974; Jenkins, Zyzanski, \& Rosenman, 1971), denotes individuals' tendency to "consider time as a scarce resource and plan its use carefully (Landy, Rastegary, Thayer, \& Colvin, 1991:645)." Time urgent individuals constantly feel the pressure of time and tend to conduct activities (e.g. work, eat, talk) in a high speed. Although prone to minor health issues such as headache, digestion problems, and respiratory problems (Conte, Mathieu, \& Landy, 1998), they are punctual to work (Dishon-Berkovits \& Koslowsky, 2002) and deliver aboveaverage job performance, particularly when the jobs are intrinsically timedemanding (Greenberg, 2002). Time urgent undergraduates with a scheduling habit achieve above-average Grade Point Average (GPA) (Conte et al., 1998). When working in groups, time urgent individuals drive their groups to complete tasks one by one, which, in turn, results in high team performance (Waller et al., 1999). Overall, the extant research suggests a positive effect of individuals' and teams' time urgency on their performance, if the time urgency measurement contains time controlling behaviors, such as scheduling and prioritizing; time urgent feeling itself does not necessarily leads to high performance.

Previous research suggests that time urgency is a multidimensional construct, whose dimensionality partially depends on the construct's operationalizations (Landy et al., 1991). Measured by a Likert-point scale developed by Landy and colleagues (1991), time urgency demonstrates five subconstructs ${ }^{10}$, including eating behavior, competitiveness, general hurry, task-related hurry, and speech pattern

\footnotetext{
${ }^{10}$ In this dissertation, "subconstruct", "dimension", and "component" are interchangeably used to indicate a first-order construct that reflects or forms a second-order multidimensional construct.
} 
(Conte, Ringenbach, Moran, \& Landy, 2001; Landy et al., 1991). Eating behavior refers to how fast an individual ${ }^{11}$ eats meals or food in general; competitiveness refers to the degree of an individual being ambitious and hard-driving, and desiring to excel; general hurry refers to an individual's general feeling of being pressured by time; task-related hurry refers to how fast an individual works and conducts activities; speech pattern refers to the speed, volume, and persuasiveness of an individual's speaking.

Measured by a Behaviorally Anchored Rating Scales (BARS) developed by Landy and colleagues (1991), time urgency contains five subconstructs, including eating behavior, awareness of time, scheduling, list making, and deadline control (Conte, Landy, \& Mathieu, 1995; Landy et al., 1995). Two subconstructs, namely, speech pattern and nervous energy, emerge in Landy and colleagues' (1991) original study but not in Conte and colleagues' (1995) later study. Both measures contain the eating behavior and speech pattern subconstruct; awareness of time in the later measure shares a similar definition as general hurry's in the former measure. Scheduling refers to "the extent to which an individual schedules activities and keeps to that schedule (Landy et al., 1991: 649);" list making refers to "the extent to which a person creates or maintains a list of things to do during the day or during the week (Landy et al., 1991: 649);" deadline control refers to "the extent to which an individual creates or appears to be controlled by external deadlines (Landy et al., 1991: 649);" nervous energy refers to "the extent to which a person can be characterized as being in constant motion, even while resting (Landy et al., 1991: 649)."

Previous research also suggests that time urgency is a personality trait. Landy and colleagues (1991) test the stability of time urgency, measured by the 33-item Likert-point scale, in a sample of 132 clerical workers over a four-month interval and report that the test-retest reliability coefficients are above 0.90 for four time urgency subconstructs, including eating behaviors, competitiveness, general hurry, and task-related hurry, and 0.70 for the fifth component, speech pattern. Measured by the BARS and tested with 168 undergraduates across four weeks, time urgency gives test-retest reliability coefficients ranging from 0.68 to 0.86 on six subconstructs in the test, including, eating behaviors, speech patterns, awareness of time, scheduling, list making, and nervous energy.

Polychronicity. Polychronicity refers to individuals' preference to perform multiple activities at the same time (Hall, 1983). High polychronicity, or low monochronicity, indicates a preference to multitask; low polychronicity, or high monochronicity, on the other hand, implies a preference to conduct multiple activities one after another. Bluedorn, Kalliath, Strube, and Martin (1998) further suggest that individuals' polychronic preferences aggregated to the collective level form a polychronic value. Empirical studies have shown inconsistent effects of polychronicity on performance. On the one hand, individual-level polychronicity is found to be

\footnotetext{
${ }^{11}$ In Chapter 2 and 3, unless explicitly indicated, "individual" refers exclusively to an individual person and excludes the notion of an individual team.
} 
positively related to faculty members' publication productivity and sales representatives' performance (Conte \& Gintoft, 2005; Taylor, Locke, Lee, \& Gist, 1984). Corporate polychronic culture is associated with higher financial performance than monochronic culture (Onken, 1999). On the other hand, researchers have reported a negative or null relationship between polychronicity and performance at the individual level (Conte \& Jacobs, 2003; Konig, Buhner, \& Murling, 2005). In addition, person-job fit in polychronicity is found to be positively related to both employees' job performance and their psychological well-being, including job satisfaction, organizational commitment, and perceived fairness (Hecht \& Allen, 2005; Madjar \& Oldham, 2006; Slocombe \& Bluedorn, 1999).

Polychronicity has not been concluded as a personality trait in previous research. Bluedorn and colleagues (1998) report a 0.89 test-retest reliability coefficient for the construct measured by a 10-item Likert-point Inventory of Polychronic Values (IPV), in a sample of 23 managers over two weeks, and 0.78 in a sample of 21 managers over four weeks. In their study, polychronicity is tested as a collective value rather than an individual preference.

Time perspective. Time perspective indicates "the totality of an individual's views of his [or her] psychological future and psychological past existing at a given time (Lewin, 1951, quoted in Zimbardo \& Boyd, 1999: 1271)." It is "a nonconscious process whereby the continual flows of personal and social experiences are assigned to temporal categories, or time frames, that help to give order, coherence, and meaning to those events (Zimbardo \& Boyd, 1999:1271)." Previous research suggests a positive relationship between future time perspective and socially desirable behaviors and a negative relationship between present time perspective and socially undesirable behaviors. For example, future orientation, a cognitive focus on the future, is found to be associated with self-efficacy, solution-seeking for predicaments, and commitment to signed-up research (Epel, Bandura, \& Zimbardo, 1999; Harber, Zimbardo \& Boyd, 2003; Keough, Zimbardo, \& Boyd, 1999). On the contrary, present orientation, a cognitive focus on present enjoyment rather future achievement, is found to be associated with substance use for stress-coping (Keough et al., 1999; Wills, Sandy, \& Yaeger, 2001). Few studies on time perspective are conducted in organizational settings, except for Das' (1987) study, which reports a positive relationship between senior managers' time perspective and their planning range.

Previous research suggests that time perspective is a multidimensional construct that contains five subconstructs (Zimbardo \& Boyd, 1999), including pastpositive, past-negative, present-hedonistic, present-fatalistic, and future orientation. Past-positive denotes a warm sentimental attitude towards his or her personal and collective past. Past-positive individuals feel pleasant to think about the past and become nostalgic about childhood. Past-negative, on the contrary, denotes a negative aversive view of the past. Past-negative individuals often think about bad things that have happened in the past, good things that have missed out in life, and what 
should have been done differently in life. Present-hedonistic denotes "a hedonistic, risk-taking, 'devil may care' attitude toward time and life (Zimbardo \& Boyd, 1999: 1275)." Present-hedonistic individuals enjoy taking risks and often act impulsively. Present-fatalistic denotes "a fatalistic, helpless, and hopeless attitude toward the future and life (Zimbardo \& Boyd, 1999: 1275)." Present-fatalistic individuals believe that life is controlled by forces outside their influence, the future cannot be planned, and luck pays off better than hard work. Future orientation is a general focus on the future. Future-oriented individuals tend to resist temptations for work and complete projects on time by making steady progress.

Time perspective has been concluded as a personality trait in previous research. Zimbardo and Boyd (1999) test the stability of time perspective, measured by a 56-item Zimbardo Time Perspective Inventory (ZTPI), among 58 students over a four-week period, and report that test-retest reliability coefficients range from 0.70 to 0.80 for the five time perspective components, that is, past-positive, pastnegative, present-hedonistic, present-fatalistic, and future orientation. Two unpublished studies report that the test-retest reliability coefficients for present-hedonistic, present-fatalistic, and future orientation, measured by the ZTPI, range between 0.70 and 0.83 over a period of four weeks and of sixteen months respectively (Jourdan, 1993; Keough, 1993, cited in Keough et al., 1999). Some researchers (Lennings, 1991; Poole \& Cooney, 1987), using an alternative measure of time perspective, report that the test-retest reliability coefficients of personal past, societal past, personal future, and societal future range from 0.58 and 0.86 over two weeks and from 0.76 to 0.84 over four months.

Temporal depth. Temporal depth is "the temporal distance into the past and future that individuals and collectivities typically consider when contemplating events that have happened, may have happened, or may happen (Bluedorn, 2002: 114)." Whereas time perspective defines a qualitative focus on the past, present, or future, temporal depth defines quantitative extensions into the past and future. According to Bluedorn (2002), temporal depth is implicitly embedded in daily languages and can be measured by asking individuals to specify how much time they indicate by such vague phrases as "short-term future", "mid-term future", "longterm future", "recently", "a middling time ago", and "a long time ago" (Bluedorn, 2002). Extant empirical evidence suggests that future temporal depth is longer than past temporal depth (Bluedorn, 2002) and that the longer people think about the past, the longer they would think about the future, but not vice versa (El Sway, 1983). Few studies, except for the aforementioned Das' (1987) study, have examined the impact of temporal depth on job performance or other organizational behaviors. To our knowledge, no empirical study in the organizational behavior literature has examined the intertemporal stability of temporal depth. 


\section{Methods}

\section{Design, Procedure, and Sample}

In order to test the intertemporal stability of time urgency, polychronicity, time perspective, temporal depth, and Big Five personality traits, we repeatedly measured these traits three times (referred to as $\mathrm{T}_{1}, \mathrm{~T}_{2}$, and $\mathrm{T}_{3}$ in the coming sections) over a period of six months. Three identical online questionnaires were made available to the faculty members, administrative staff, and students in the Faculty of Economics and Business Administration ${ }^{12}$ of Maastricht University in the Netherlands at the end of December 2006, March 2007, and June 2007. The three questionnaires were administered in the same week of three consecutive academic quarters $^{13}$ (i.e. the second-to-last week). Each time, invitation emails were sent to the potential participants via the faculty's internal emailing system, which contained links to the questionnaires. In order to encourage participants' continuous participation in the study, we rewarded one participant among those who completed all the three questionnaires in the end with a 200 -Euro cash prize ${ }^{14}$. The prize winner was generated through a lottery procedure of random selection. We reminded potential respondents of this rewarding policy in the three wave invitation emails.

121 respondents completed the first online questionnaire, 75 of them the second one, and 63 the last one. We presented all the questionnaires in English as in the original journal articles. We assumed adequate English proficiency of the potential respondents, since all the educational programs taught in the faculty-except for one course in the Fiscal Economics program-were offered in English. In addition, the highly internationalized target sample-around $40 \%$ of the enrolled students and 33\% staff members were non-Dutch citizens and of 66 nationalitiesmade it impractical to translate the questionnaires into the native language of every potential respondent.

\section{Measures}

We measured the four temporal characteristics by previously published instruments. The repeated measurement design and lengthy overall questionnaires might discourage participants' from continuously participating in the study, which prompted us to shorten the length of some measurement instruments. Hence, we selected questionnaire items of the dimensions of time urgency and time perspective by the following two criteria. First, each item must load on one unique dimension

\footnotetext{
${ }^{12}$ The faculty has changed its name to the School of Business and Economics in May, 2009. .

${ }^{13}$ The academic year of the faculty consists of four eight-week academic quarters and two two-week skill training periods. Every half year, two academic quarters precede one skill training period. One academic quarter consists of seven educational weeks and one exam week.

${ }^{14}$ The faculty's policy does not allow researchers to directly give cash to research participants. Therefore, we rewarded all the prize winners in the dissertation studies with shopping voucher of equivalent values, which they could use in most local stores.
} 
with an above- 0.50 factor loading in the original study. Second, at least three items are present for each dimension. These selection criteria ensure the inclusion of the most robust items and take into account the trade-off between the questionnaire's length and participants' continuous participation to a longitudinal study. The measurement is presented in Appendix A (Scale 1 to 5).

Time urgency. Time urgency was measured by $17^{15}$ five-point Likert items selected from the 33-item scale in Landy and colleagues' study (1991). The criteria for item selection resulted in 17 items for eating behaviors, competitiveness, general hurry, and task-related hurry. The fifth subconstruct, speech pattern, was excluded, because no item had an above- 0.5 factor loading in Landy and colleagues' study (1991). The selected items are, for example, "my spouse and close friends told me that I eat too fast," "I am competitive and hard-driving," "I usually feel much pressured by time," and "I usually work fast and energetically." We asked each respondent to indicate how well each item described him or her from the choices provided in the scale.

Polychronicity. Polychronicity was measured by the four-item five-Likertpoint Polychronic Attitudes Inventory (PAI, Bluedorn, Kaufman, \& Lane, 1992), including such items as "I do not like juggle several activities at the same time (R)," "People should not try to do many things at once (R)," "When I sit down at my desk, I work on one project at a time (R)," and "I am comfortable doing several things at the same time." The letter $\mathrm{R}$ in the brackets indicates reverse wording. We asked each respondent to what degree he or she agreed with each item.

Time perspective. Time perspective was measured by $22^{16}$ five-point Likert items selected from the 56-item ZTPI (Zimbardo \& Boyd, 1999) according to the two aforementioned criteria. The selected items measured all the five time perspective subconstructs, including past-positive, past-negative, present-hedonistic, present-fatalistic, and future-orientation. These items are, for example, "Familiar childhood signs, sounds, smells often bring back a flood of wonderful memories," "It is hard for me to forget unpleasant images of my youth," "It is important to put excitement in my life," "My life path is controlled by forces I cannot influence," and "Meeting tomorrow's deadlines and doing other necessary work comes before tonight's play." We asked each respondent how well each item described him or her.

Temporal depth. We measured temporal depth by asking each respondent to specify how much exact time he or she refers to "short term future," "mid-term future," "long-term future," "recently," "a middling time ago," and "a long time ago" (Bluedorn, 2002). Fifteen choices were offered for each phrase, including one day, one week, two weeks, one month, three months, six months, nine months, one

\footnotetext{
${ }^{15}$ One item of competitiveness item was excluded from the stability test, because of the small sample size $(\mathrm{n}=63)$ in the study.

${ }^{16}$ One item of past-positive was excluded from the stability test, because of the small sample size $(n=63)$.
} 
year, three years, five years, ten years, fifteen years, twenty years, twenty-five years, and more than twenty-five years. When analyzing the data, we coded the choices of one day, one week, and two weeks as 1 , one month and three months 2 , six months and nine months 3 , one year and three years 4 , five years and ten years 5 , fifteen years and twenty years 6 , and twenty-five years and more than twenty-five years 7 .

\section{Analyses}

In the preliminary analyses, we examined the randomness of missing data by comparing the $T_{1}$ score differences of each temporal characteristic between the all-time committed respondents and respondents dropped out at $\mathrm{T}_{2}$ and between the all-time committed respondents and respondents dropped out at $\mathrm{T}_{3}$. If data missing was found to be related to a temporal characteristic, it implied that this characteristic may lead to participant dropout and that the distribution of the 63 remaining participants' attribute levels may not represent the distribution at the sample level. We would then exclude it from further stability testing.

To test the intertemporal stability of the four temporal characteristics, we used the data from the 63 respondents who participated in the study at all the three time moments. We performed the LGM procedure that is used in Lance and colleagues' (2000) studies for each dimensions of time urgency and time perspective, and polychronicity, because each construct was measured by multiple items. We performed repeated-measures multivariate analysis of variance (MANOVA) to test the intertemporal stability of temporal depth, because each temporal depth subconstruct (i.e. short-, mid-, and long-term past temporal depth, and short-, mid-, and longterm future temporal depth) was measured by one item.

The LGM procedure, performed with the LISREL software, consisted of the tests of six progressively restricted models and an additional model that tests the measurement equivalence for each construct across three time moments. Systematically testing the nested models allows us to identify the occurrence of construct validity change (i.e. gamma change), construct calibration change (i.e. beta change), and construct score change (i.e. alpha change). If and only if, the measurement equivalence of a construct was established, as indicated by the absence of gamma and beta change, we could further test the equivalence of sample distribution and sample mean of the construct score. We elaborate the testing procedure as below and present the syntaxes with 'eating behavior' as an example in Appendix B.

In the first model (M1) we assumed that each item loaded exclusively on its hypothetical construct across the three time moments, error terms of each item covaried between two time moments (i.e. between $T_{1}$ and $T_{2}, T_{2}$ and $T_{3}, T_{1}$ and $T_{3}$ ), item intercepts and factor loadings of all items were free to estimate-except that the factor loading of the first item was fixed to 1 - and factor covariances and factor variances were free to estimate. In our terminology, "one factor" is one construct 
at one time moment; "between-factor" means one construct between two time moments. The general tested model is written as

$$
\mathrm{x}(\mathrm{kit})=\alpha(\mathrm{kt})+\lambda(\mathrm{kt}) * \xi(\mathrm{it})+\varepsilon(\mathrm{kit})
$$

in which $\mathrm{x}$ is the item score, $\alpha$ the item intercept on the latent factor, $\lambda$ the factor loading, $\xi$ the latent factor, $\varepsilon$ the error term, $\mathrm{k}$ the item, $\mathrm{i}$ the individual, and $\mathrm{t}$ the time moment. The restriction of M1 was $\xi(\mathrm{i} 1)=\xi(\mathrm{i} 2)=\xi(\mathrm{i} 3)$. Rejection of M1 implied that a construct did not have an identical factor structure across the three time moments and that gamma change occurred.

In the second model (M2) we assumed, in addition to the identical item-factor structure over time, identical factors loading of each item on the latent factor across the three time moments. The additional restriction was $\lambda(\mathrm{k} 1)=\lambda(\mathrm{k} 2)=\lambda(\mathrm{k} 3)$. The identical factor loading of each item was fixed to the mean factor loading averaged across the three loadings estimated in M1. Rejection of M2 also implied the occurrence of gamma change.

In the third model (M3) we assumed, in addition to the above two assumptions, that the item intercept of each item on the latent factor was equal across the three time moments. The additional restriction was $\alpha(\mathrm{k} 1)=\alpha(\mathrm{k} 2)=\alpha(\mathrm{k} 3)$. The identical item intercept of each item was fixed to the mean item intercept averaged across the three item intercepts estimated in M2. Rejection of M3 implied the occurrence of beta change.

In the fourth model (M4) we assumed, in addition to the above three assumptions, that the error variance of each item was equal across the three time moments. The additional restriction was $\sigma_{\varepsilon}(\mathrm{k} 1)=\sigma_{\varepsilon}(\mathrm{k} 2)=\sigma_{\varepsilon}(\mathrm{k} 3)$. Error variance represents the variance of an item not explained by the latent factor structure. Rejection of M4 also implied the occurrence of beta change.

In the fifth model (M5) we assumed, in addition to the above four assumptions, that the factor variance of a construct was equal across the three time moments. The additional restriction was $\sigma_{\xi 1}=\sigma_{\xi 2}=\sigma_{\xi 3}$. Factor variance represents the dispersion of individuals' construct scores in the sample. Rejection of M5 implied that individuals' relative positions, or rank orders of the construct scores in the sample changed over time and that alpha change occurred.

In the sixth model (M6) we assumed, in addition to the above five assumptions, that the factor covariance of a construct was equal between at any two of the three time moments (i.e. at $\mathrm{T}_{1}$ and $\mathrm{T}_{2}, \mathrm{~T}_{2}$. and $\mathrm{T}_{3}, \mathrm{~T}_{1}$ and $\mathrm{T}_{3}$ ). The additional restriction was $\sigma_{\xi 1 \xi 2}=\sigma_{\xi 2 \xi 3}=\sigma_{\xi 1 \xi 3}$. The identical factor covariance was fixed to the mean factor covariance averaged across the three estimated factor covariances in M5. Rejection of M6 implied the occurrence of alpha change.

In the seventh model (M7) we assumed, in addition to the above six assumptions, that factor mean was equivalent across the three time moments. The additional restriction was $\mathrm{E}(\xi 1)=\mathrm{E}(\xi 2)=\mathrm{E}(\xi 3)$. We calculated the factor mean based on 
the function $\mathrm{x}(\mathrm{kt})=\alpha(\mathrm{kt})+\lambda(\mathrm{kt}) * \xi(\mathrm{t})$, in which $\mathrm{x}(\mathrm{kt})$ is the item mean, $\alpha$ the identical item intercept estimated in $\mathrm{M} 3$, and $\lambda$ the identical factor loading estimated in M2. We first calculated a latent factor score for each item at each time moment by subtracting the item intercept and then dividing by the factor loading. We then calculated the grand factor mean by averaging the latent factor scores across items. We fixed the factor mean at each time moment to the grand mean. Rejection of M7 implied that the sample mean of the construct score changed over time and that alpha change occurred. In contrast, acceptance of M1 to M7 implied that the sample mean of the construct score was stable over time. The real construct score stability is the stability of sample mean score, if and only if adequate equivalence of construct validity, construct calibration, and within-sample distribution of construct score is pre-established.

We assessed model fit by Chi-squares, comparative fit index (CFI), and root mean squared error of approximation (RMSEA). An above .95 CFI and a below .05 RMSEA (Bollen, 1989) indicate a good model fit. We also compared the differences of Chi-square between two adjacent progressively restricted models in order to identify which change - gamma, beta, or alpha change - occurred for each construct.

\section{Results}

The preliminary analysis results suggest that respondent dropout was related to present-fatalistic, a subconstruct of time perspective, and long-term future temporal depth. At $\mathrm{T}_{1}$, the all-time committed respondents felt less fatalistic about the present $(\mathrm{F}=2.104, \mathrm{p}<0.1)$ and had deeper long-term future temporal depth $(\mathrm{F}=3.416, \mathrm{p}<$ $0.05)$ than did those who later dropped out from the study. We excluded the two subconstructs from further analyses of construct intertemporal stability.

The LGM analysis results suggest that for time urgency, construct validity change (i.e. gamma change) occurred for eating behavior (ET), general hurry (GH) and task-related hurry $(\mathrm{TRH})$, since $\mathrm{M} 1$ was rejected $\left(\mathrm{CFI}_{\mathrm{ET}}=.98, \mathrm{RMSEA}_{\mathrm{ET}}=.07\right.$; $\left.\mathrm{CFI}_{\mathrm{GH}}=.98, \mathrm{RMSEA}_{\mathrm{GH}}=.11 ; \mathrm{CFI}_{\mathrm{TRH}}=.98, \mathrm{RMSEA}_{\mathrm{TRH}}=.10\right)$ and construct calibration change (i.e. alpha change) occurred in competitiveness (CP), since M4 was rejected $\left(\mathrm{CFI}_{\mathrm{CP}}=.94, \mathrm{RMSEA}_{\mathrm{CP}}=.05\right)$ and the Chi-square difference was significant between M2 and M3 $(\Delta \chi 2[15]=22.46, p<.10)$. For polychronicity, construct validity change (i.e. gamma change) occurred, since M1 was rejected (CFI=.97, RMSEA=.06). For time perspective, construct validity change (i.e. gamma change) occurred for past-positive (PP), past-negative (PN), and present-hedonistic ( $\mathrm{PH})$, since M1 was rejected $\left(\mathrm{CFI}_{\mathrm{PP}}=.96, \mathrm{RMSEA}_{\mathrm{PP}}=.08 ; \mathrm{CFI}_{\mathrm{PN}}=.96, \mathrm{RMSEA}_{\mathrm{PN}}=.09\right.$ $\mathrm{CFI}_{\mathrm{PH}}=.99, \mathrm{RMSEA}_{\mathrm{PH}}=.06$ ). Future orientation, on the other hand, is the most stable dimension of time perspective, demonstrating no change in construct validity, construct calibration, or construct mean over time and the acceptance of M1 to M7. Its 
estimated equivalent mean over time is 3.79 on the 1-to-5 scale. For temporal depth, repeated-measures MANOVA results indicate a lack of within-subjects effect for short- and mid-term future temporal depth, and for short-, mid- and long-term past temporal depth respectively. We present the analysis results in Table 2.1 to Table 2.4 and summarize the results in the alpha-beta-gamma-change typology in Table 2.5 .

Table 2.1The LGM Results of the Intertemporal Stability of Time Urgency $(n=63)$

\begin{tabular}{|c|c|c|c|c|c|c|c|c|}
\hline \multirow[t]{2}{*}{ Model } & \multicolumn{4}{|c|}{ Eating behavior } & \multicolumn{4}{|c|}{ Competitiveness } \\
\hline & $\mathrm{df}$ & $\chi^{2}$ & RMSEA & CFI & $\mathrm{df}$ & $\chi^{2}$ & RMSEA & CFI \\
\hline $\begin{array}{l}\text { 1. Equivalent } \\
\text { factor structure }\end{array}$ & 72 & 94.83 & .07 & .98 & 72 & 82.30 & .05 & .96 \\
\hline 1 vs. 2 & 12 & 8.24 & -- & -- & 12 & 7.39 & -- & -- \\
\hline $\begin{array}{l}\text { 2. Equal factor } \\
\text { loadings }\end{array}$ & 84 & 103.07 & .06 & .98 & 84 & 89.69 & .03 & .97 \\
\hline 2 vs. 3 & 15 & 3.3 & -- & -- & 15 & $22.46^{*}$ & -- & -- \\
\hline $\begin{array}{l}\text { 3. Equal item } \\
\text { intercepts }\end{array}$ & 99 & 106.37 & .04 & .99 & 99 & 112.15 & .05 & .96 \\
\hline 3 vs.4 & 10 & 10.22 & -- & -- & 10 & 15.36 & -- & -- \\
\hline $\begin{array}{l}\text { 4. Equal error } \\
\text { variances }\end{array}$ & 109 & 116.59 & .03 & .99 & 109 & 127.51 & .05 & .94 \\
\hline 4 vs. 5 & 2 & 1.25 & -- & -- & 2 & 0.45 & -- & -- \\
\hline $\begin{array}{l}\text { 5. Equal factor } \\
\text { variances }\end{array}$ & 111 & 117.84 & .03 & .99 & 111 & 127.96 & .05 & .94 \\
\hline 5 vs. 6 & 3 & 5.93 & -- & -- & 3 & 0.58 & -- & -- \\
\hline $\begin{array}{l}\text { 6. Equal factor } \\
\text { covariances }\end{array}$ & 114 & 123.77 & .04 & .99 & 114 & 128.54 & .05 & .94 \\
\hline 6 vs. 7 & 0 & .00 & -- & -- & 0 & 0.16 & -- & -- \\
\hline $\begin{array}{l}\text { 7. Equal factor } \\
\text { means }\end{array}$ & 114 & 123.77 & .04 & .99 & 114 & 128.70 & .05 & .94 \\
\hline
\end{tabular}

Note. From Table 2.1 to Table 2.4 and from Table 3.1 to Table 3.4. $* p<.10 . * * p<.05 . * * * p<.01$. 
Table 2.1 (continued)

\begin{tabular}{|c|c|c|c|c|c|c|c|c|}
\hline \multirow[t]{2}{*}{ Model } & \multicolumn{4}{|c|}{ General hurry } & \multicolumn{4}{|c|}{ Task-related hurry } \\
\hline & $\mathrm{df}$ & $\chi^{2}$ & RMSEA & CFI & $\mathrm{df}$ & $\chi^{2}$ & RMSEA & CFI \\
\hline $\begin{array}{l}\text { 1. Equivalent factor } \\
\text { structure }\end{array}$ & 15 & 26.34 & .11 & .98 & 15 & 24.27 & .10 & .98 \\
\hline 1 vs. 2 & 6 & -0.07 & -- & -- & 6 & 2.35 & -- & -- \\
\hline $\begin{array}{l}\text { 2. Equal factor loa- } \\
\text { dings }\end{array}$ & 21 & 26.27 & .06 & .99 & 21 & 26.62 & .07 & .99 \\
\hline 2 vs. 3 & 9 & 17.04 & -- & -- & 9 & 7.97 & -- & -- \\
\hline $\begin{array}{l}\text { 3. Equal item inter- } \\
\text { cepts }\end{array}$ & 30 & 43.31 & .09 & .98 & 30 & 34.59 & .05 & .99 \\
\hline 3 vs. 4 & 6 & 11.80 & -- & -- & 6 & 9.74 & -- & -- \\
\hline $\begin{array}{l}\text { 4. Equal error varian- } \\
\text { ces }\end{array}$ & 36 & 55.11 & .09 & .96 & 36 & 44.33 & .06 & .98 \\
\hline 4 vs. 5 & 2 & 2.1 & -- & -- & 2 & -0.05 & -- & -- \\
\hline $\begin{array}{l}\text { 5. Equal factor varian- } \\
\text { ces }\end{array}$ & 38 & 57.21 & .09 & .96 & 38 & 44.28 & .05 & .99 \\
\hline 5 vs. 6 & 3 & 1.89 & -- & -- & 3 & 1.41 & -- & -- \\
\hline $\begin{array}{l}\text { 6. Equal factor covari- } \\
\text { ances }\end{array}$ & 41 & 59.10 & .08 & .97 & 41 & 59.31 & .09 & .97 \\
\hline 6 vs. 7 & 0 & -0.02 & -- & -- & 0 & -0.01 & -- & -- \\
\hline 7. Equal factor means & 41 & 59.09 & .08 & .97 & 41 & 59.30 & .09 & .97 \\
\hline
\end{tabular}


Table 2.2 The LGM Results of the Intertemporal Stability of Polychronicity $(n=63)$

\begin{tabular}{lcccc}
\hline \multirow{1}{*}{ Model } & \multicolumn{4}{c}{ Polychronicity } \\
\cline { 2 - 5 } & $\mathrm{df}$ & $\chi^{2}$ & RMSEA & CFI \\
\hline 1. Equivalent factor structure & 39 & 48.67 & .06 & .97 \\
1 vs. 2 & 9 & 4.19 & -- & -- \\
2. Equal factor loadings & 48 & 52.86 & .04 & .97 \\
2 vs. 3 & 12 & 10.31 & -- & -- \\
3. Equal item intercepts & 60 & 63.17 & .03 & .97 \\
3 vs.4 & 8 & 17.55 & -- & -- \\
4. Equal error variances & 68 & 80.72 & .06 & .96 \\
4 vs.5 & 2 & 4.93 & -- & -- \\
5. Equal factor variances & 70 & 85.65 & .06 & .96 \\
5 vs. 6 & 3 & 0.03 & -- & -- \\
6. Equal factor covariances & 73 & 85.68 & .05 & .96 \\
6 vs.7 & 0 & 0 & -- & -- \\
7. Equal factor means & 73 & 85.68 & .05 & .96 \\
\hline
\end{tabular}


Table 2.3 The LGM Results of the Intertemporal Stability of Time Perspective $(n=63)$

\begin{tabular}{|c|c|c|c|c|c|c|c|c|}
\hline \multirow[t]{2}{*}{ Model } & \multicolumn{4}{|c|}{ Past-positive } & \multicolumn{4}{|c|}{ Past-negative } \\
\hline & $\mathrm{df}$ & $\chi^{2}$ & RMSEA & CFI & $\mathrm{df}$ & $\chi^{2}$ & RMSEA & CFI \\
\hline $\begin{array}{l}\text { 1. Equivalent } \\
\text { factor structure }\end{array}$ & 72 & 101.04 & .08 & .96 & 72 & 104.63 & .09 & .96 \\
\hline 1 vs. 2 & 12 & 14.87 & -- & -- & 12 & 9.38 & -- & -- \\
\hline $\begin{array}{l}\text { 2. Equal factor } \\
\text { loadings }\end{array}$ & 84 & 115.91 & .08 & .96 & 84 & 114.01 & .08 & .96 \\
\hline 2 vs. 3 & 15 & 7.9 & -- & -- & 15 & 19.95 & -- & -- \\
\hline $\begin{array}{l}\text { 3. Equal item } \\
\text { intercepts }\end{array}$ & 99 & 123.81 & .06 & .96 & 99 & 133.96 & .08 & .95 \\
\hline 3 vs. 4 & 10 & 10.12 & -- & -- & 10 & 6.74 & -- & -- \\
\hline $\begin{array}{l}\text { 4. Equal error } \\
\text { variances }\end{array}$ & 109 & 133.93 & .06 & .96 & 109 & 140.70 & .07 & .95 \\
\hline 4 vs. 5 & 2 & 0.84 & -- & -- & 2 & 2.1 & -- & -- \\
\hline $\begin{array}{l}\text { 5. Equal factor } \\
\text { variances }\end{array}$ & 111 & 134.77 & .06 & .96 & 111 & 142.80 & .07 & .95 \\
\hline 5 vs. 6 & 3 & 0.69 & -- & -- & 3 & $9.54 * *$ & -- & -- \\
\hline $\begin{array}{l}\text { 6. Equal factor } \\
\text { covariances }\end{array}$ & 114 & 135.46 & .06 & .96 & 114 & 152.34 & .07 & .95 \\
\hline $6 \mathrm{vs} .7$ & 0 & 0.02 & -- & -- & 0 & 0 & -- & -- \\
\hline $\begin{array}{l}\text { 7. Equal factor } \\
\text { means }\end{array}$ & 114 & 135.48 & .06 & 096 & 114 & 152.34 & .07 & .95 \\
\hline
\end{tabular}


Table 2.3 (continued)

\begin{tabular}{|c|c|c|c|c|c|c|c|c|}
\hline \multirow[t]{2}{*}{ Model } & \multicolumn{4}{|c|}{ Present-hedonistic } & \multicolumn{4}{|c|}{ Future-oriented } \\
\hline & $\mathrm{df}$ & $\chi^{2}$ & RMSEA & CFI & $\mathrm{df}$ & $\chi^{2}$ & RMSEA & CFI \\
\hline $\begin{array}{l}\text { 1. Equivalent } \\
\text { factor structure }\end{array}$ & 15 & 18.38 & .06 & .99 & 39 & 42.14 & .04 & .99 \\
\hline 1 vs. 2 & 6 & 1.51 & -- & -- & 9 & 7.29 & -- & -- \\
\hline $\begin{array}{l}\text { 2. Equal factor } \\
\text { loadings }\end{array}$ & 21 & 19.89 & .00 & 1.00 & 48 & 49.43 & .02 & .98 \\
\hline 2 vs. 3 & 9 & 6.16 & -- & -- & 12 & 3.95 & -- & -- \\
\hline $\begin{array}{l}\text { 3. Equal item } \\
\text { intercepts }\end{array}$ & 30 & 26.05 & .00 & 1.00 & 60 & 53.38 & .00 & 1.00 \\
\hline 3 vs. 4 & 6 & $18.54 * * *$ & -- & -- & 8 & 8.48 & -- & -- \\
\hline $\begin{array}{l}\text { 4. Equal error } \\
\text { variances }\end{array}$ & 36 & 44.59 & .06 & .99 & 68 & 61.86 & .00 & .99 \\
\hline 4 vs. 5 & 2 & 1.17 & -- & -- & 2 & 5.17 & -- & -- \\
\hline $\begin{array}{l}\text { 5. Equal factor } \\
\text { variances }\end{array}$ & 38 & 45.76 & .06 & .99 & 70 & 67.03 & .00 & .99 \\
\hline 5 vs. 6 & 3 & 0.46 & -- & -- & 3 & 1.05 & -- & -- \\
\hline $\begin{array}{l}\text { 6. Equal factor } \\
\text { covariances }\end{array}$ & 41 & 46.22 & .06 & .99 & 73 & 68.08 & .00 & .99 \\
\hline 6 vs. 7 & 0 & 3.89 & -- & -- & 0 & -0.02 & -- & -- \\
\hline $\begin{array}{l}\text { 7. Equal factor } \\
\text { means }\end{array}$ & 41 & 50.11 & .06 & .99 & 73 & 68.06 & .00 & .99 \\
\hline
\end{tabular}


Table 2.4 The Repeated-Measures MANOVA Results of the Intertemporal Stability of Temporal Depth $(n=63)$

\begin{tabular}{ccccccccccc}
\hline Source & $\begin{array}{c}\text { Short-team } \\
\text { FTD }\end{array}$ & \multicolumn{2}{c}{$\begin{array}{c}\text { Mid-term } \\
\text { FTD }\end{array}$} & \multicolumn{2}{c}{$\begin{array}{c}\text { Short-term } \\
\text { PTD }\end{array}$} & \multicolumn{2}{c}{$\begin{array}{c}\text { Mid-term } \\
\text { PTD }\end{array}$} & \multicolumn{2}{c}{$\begin{array}{c}\text { Long-term } \\
\text { PTD }\end{array}$} \\
\cline { 2 - 13 } & $\mathrm{F}$ & $\mathrm{df}$ & $\mathrm{F}$ & $\mathrm{df}$ & $\mathrm{F}$ & $\mathrm{df}$ & $\mathrm{F}$ & $\mathrm{df}$ & $\mathrm{F}$ & $\mathrm{df}$ \\
\hline Time & 2.020 & 2 & 1.988 & 2 & 2.267 & 2 & 0.855 & 2 & 0.163 & 2 \\
\hline
\end{tabular}

Note. ${ }^{\text {a }}$ The reported F-statistics are those when sphericity is assumed. FTD $=$ future temporal depth. PTD = past temporal depth. None of the F-statistics is significant.

Table 2.5 Summary of Test Results of the Intertemporal Stability of Time Urgency, Polychronicity, Time Perspective, and Temporal Depth $(n=63)$

\begin{tabular}{l|ccc}
\hline \multicolumn{1}{c|}{ Temporal characteristic } & $\gamma$ change & $\beta$ change & $\alpha$ change \\
\hline Time urgency & $\sqrt{ }$ & -- & -- \\
Eating behaviors & -- & $\sqrt{ }$ & -- \\
Competitiveness & $\sqrt{ }$ & -- & -- \\
General hurry & $\sqrt{ }$ & -- & -- \\
Task-Related hurry & $\sqrt{ }$ & & -- \\
Polychronicity & $\sqrt{ }$ & -- & -- \\
Time perspective & $\sqrt{ }$ & -- & - \\
Past-Positive & $\sqrt{ }$ & -- & -- \\
Past-Negative & -- & - & N.A. \\
Present-Hedonistic & N.A. & N.A. & \\
Future-Oriented & & & \\
Temporal depth & &
\end{tabular}

Note .N.A= Not applicable. 


\section{Discussion}

The aim of the study was to examine the intertemporal stability of time urgency, polychronicity, time perspective, and temporal depth over six months. For each construct measured by multiple questionnaire items, we tested the intertemporal stability via the alpha-beta-gamma-change typology and latent growth modeling, a methodology superior to the traditional test-retest reliability coefficient in examining whether an attribute is adequately stable over time to be a personality trait. The study yields two major findings.

First, most temporal characteristics vary in their measurement over time. In particular, eating behavior, general hurry, and task-related hurry of time urgency, past-positive, past-negative, and present-hedonistic of time perspective, and the unidimensional polychronicity vary in construct validity over time. It implies that the same set of questionnair items does not evoke the same conceptual frame of reference over time. Rather, for the same group of individuals the construct is represented by distinct sets of attitudes and behaviors over time. Consequently, it is not justified to further assess the level change of the construct over time. Competitiveness of time urgency varies in construct calibration over time. It implies that the calibration of the same set of measurement items does not provide the same calibration of the latent factor (i.e. competitiveness) over time. Likewise, it is not justified to further assess the change of competitiveness level over time. Future-orientation of time perspective and temporal depth are the most stable temporal characteristics among all, as they do not demonstrate attribute level change over time.

The results also show that the dimensions of time urgency and time perspective differ in their intertemporal stability over time. For time urgency, eating behavior, general hurry, and task-related hurry are less stable than competitiveness over time, given that the former three dimensions vary in construct validity over time and the later one demonstrates rather stable construct validity but varies in measurement calibration over time. Likewise, for time perspective, past-positive, pastnegative, and present-hedonistic are less stable than future-orientation over time, since the former three dimensions vary in measurement validity over time and the later one demonstrates rather stable measurement and attribute level over time. Lance and colleagues (2000) suggested that dimensions of a multidimensional construct have two time-related discriminant validities, that is, differential longitudinal measurement properties and differential longitudinal changes. They differ in, in addition to factorial validity (i.e. the relations to the multidimensional construct) and nomological validity (i.e. the relations to an antecedents or consequence), the intertemporal stability of measurement and the pattern of attribute level change ${ }^{17}$ over time.

\footnotetext{
${ }^{17}$ The differential patterns of attribute level change over time are not found in the study.
} 


\section{Theoretical Implications}

We draw three major implications from this study for team research that applies the IPO framework. First, time urgency, polychronicity, and time perspective should not be considered as personality traits, due to their low stability over time. The findings cast doubts on using the single-moment measure of the three temporal characteristics to predict time-related team processes (e.g. pacing, attention to time) and team outputs (e.g. productivity-by-time, meeting project deadlines). Such practice raises two particular concerns, that is, reverse causality and time-contingent prediction. Reverse causality refers to the logical ambiguity as to whether team members' temporal characteristics determine how team members perceive time and interact with each other throughout team functioning period and/or what performance results teams produce, or, vice versa, they are determined by how team members experience time and interact with each other while accomplishing team tasks and/or what time-related performance teams have produced. The reported low intertemporal stability suggests that team members' temporal characteristics are likely to be influenced by time-related team processes and team outputs. How team members have experienced time and how well teams meet intermediate deadlines may affect for example, team members' experience of time pressure (i.e. general hurry of time urgency), multitasking preference (i.e. polychronicity), and positive and negative feelings towards the past (i.e. past-positive and past-present of time perspective). Although researchers have theorized that team members' general feelings of time pressure (i.e. general hurry) may affect the likelihood of project teams meeting project deadlines (Waller et al., 2001), the low intertemporal stability of general hurry suggests that team members' experience of meeting previous project deadlines or intermediate temporal milestones of current projects may affect their general feelings of time pressure.

Furthermore, low intertemporal stability also suggests that predictions of team processes and team outputs may be contingent on the moments at which the predictors were measured. For example, the power of team members' working speed (i.e. task-related hurry of time urgency) to predict the likelihood of teams meeting project deadlines may depend on when task-related hurry is measured in relations to the progress of team projects. Variable construct validity suggests that team members may judge working speed differently (e.g. by the working speed of distinct sets of team tasks) throughout team projects. Such temporally contingent predictions of deep-level team inputs might explain their inconsistent predictions of team processes and team performance. In future research, it is important to examine the intertemporal stability of presumably trait-like attributes before using them as predictors. If intertemporal stability is found insufficient researchers may consider repeatedly measuring the deep-level team inputs and using them as time-variant predictors. 
Second, we propose to use the alpha-beta-gamma-change typology and LGM procedure to examine the intertemporal stability of temporal characteristics or other psychological attributes. The proposed methodology is superior to the traditional test-retest reliability coefficient for two aforementioned reasons. First, it decomposes change in measured attribute scores into change in measurement instrument (i.e. change in construct validity and instrument calibration) and change in attribute levels. Hence, it takes into account the possibility that change in measured attribute scores can be confounded by change in measurement instrument, rather than reflecting solely change in subjects' attribute levels. Second, it distinguishes between change in subjects' rank orders of the attribute levels over time and change in subjects' attribute levels over time. If a group of subjects change their attribute levels to the same extent over time, the proposed methodology can identify such attribute level change that is otherwise undetectable by the test-retest reliability coefficient.

Third, the finding that the dimensions of time urgency and time perspective differ in measurement equivalence over time underlines the importance of studying time-related discriminant validities in empirical longitudinal research that involves multidimensional constructs. For example, from a conceptual perspective, taskrelated hurry of time urgency may be a suitable predictor of team performance, given its high relevance to work settings. However, its low intertemporal stability (i.e. variant construct validity over time) suggests potentially inconsistent predictions of work performance if the predictor's measurement moment in relations to teams' developmental stages or life expectancy varies across studies. Therefore, in addition to a construct's conceptual content, researchers need to consider the intertemporal stability of the construct or its dimensions when empirically testing pertinent models via longitudinal research with a repeated measurement design.

\section{Managerial Implications}

Given its methodological nature, this study does not have immediate implications for managerial practice. Yet, one might consider the intertemporal stability of an attribute as an indicator of the degree to which the attribute is malleable, or in other words, responsive to environmental change under natural conditions, and, consequently, an indicator of the relative ease to change the attribute. High intertemporal stability may indicate more efforts to be required to change individuals' attribute levels over time than low intertemporal stability. For example, the reported high intertemporal stability of temporal depth in this study indicates substantial difficulty for individuals to change their cognitive temporal lengths into the past and future when retrieving relevant information for decision-making. In contrast, the reported low intertemporal stability of time urgency and time perspective subconstructs (except for future orientation) and polychronicity indicates that these temporal characteristics are open to situational influences and perhaps designed interventions. It 
may be easy for organizations to encourage individual employees to be competitive, expose them to time pressure, nurture their polychronic preference, and change their positive and negative impressions of the past, and hedonistic views of the present. Moreover, by offering suitable time management training programs, organizations might be able to adapt employees' temporal experiences to meet their job requirements and improve their working efficiency, work performance, and psychological well-beings accordingly (Hecht \& Allen, 2005; Madjar \& Oldham, 2006).

\section{Limitations}

There are several limitations in the study. First, the generalizability of the reported findings may be restricted to the particular type of population that the sample represents (i.e. university employees and students) and to the particular time interval of six months. University participants' activities are largely regulated by the rhythm of the academic calendar. Replication of the study with other sample types from different industries, societal segments, or countries is crucial for validating the reported findings. It is also crucial to replicate the study over different time intervals, in order to obtain a comprehensive picture of the intertemporal stability of the studied temporal characteristics.

Second, the validity of the reported findings is challenged by the relatively small sample size $(n=63)$. About $50 \%$ of our initial respondents dropped out from the study in the end, which signals a particular challenge of longitudinal research as such. In order to obtain a sufficiently large sample size in the end, researchers may need to draw samples from larger sample pools and employ more effective incentive schemes to encourage continuous participation.

Third, we did not examine the antecedents of the intertemporal changes of the four temporal characteristics and therefore know little what lead to the reported changes. We also know little about from which temporal interval (i.e. between $\mathrm{T}_{1}$ and $T_{2}$, or between $T_{2}$ and $T_{3}$ ) the reported changes stem from, since we only examine the overall change of each temporal characteristic across the three time moments. Furthermore, the reported findings may be confounded by other factors. For example, the questionnaires were not translated in the mother language of every respondent, which may result in the reported attribute change over time. These issues should be addressed in future research. 


\section{Chapter 3 \\ Intersituational Stability of Temporal Characteristics}

It has been theorized that team members' temporal characteristics, such as the feeling of time urgency and orientation towards the past, present, or future, will affect project teams' time-related processes (e.g. attention to time, pacing) and performance (e.g. meeting project deadlines, Waller, Conte, Gibson, \& Carpenter, 2001). In order to produce consistent predictions of team processes and team outputs, individual temporal must be stable over time and across situations. High intersituational stability indicates that team members' temporal characteristics are immune to the influence of the temporal cues in the team situation. In this study, we test the stability of time urgency, polychronicity, time perspective, and temporal depth over a period of one month in two conditions that differ in situational strength. Using a quasi-experiment, we examine the differences in the intertemporal stability of these temporal characteristics comparing an experimental condition comprising a "weak" situation and a "strong" situation, and a control condition comprising two "weak" situations. As in Chapter 2, we apply the alpha-beta-gamma-change typology (Golembiewski, Billingsley, \& Yeager 1976) and a latent growth modeling (LGM) procedure (Chan, 1998; Lance, Vandenberg, \& Self, 2000) to compare the intertemporal stability of the two conditions. Our results show that most temporal characteristics vary in measurement equivalence between the two conditions. 
In the previous study (Chapter 2) we examined the intertemporal stability of time urgency, polychronicity, time perspective, and temporal depth over a period of six months in a naturalistic condition and reported that most temporal characteristics show a change in construct validity over time. The exceptions are the competitiveness dimension of time urgency, which changes in measurement calibration over time, and future-orientation of time perspective and temporal depth, which are stable in attribute level over time. In the present study, we extend the stability research by examining the intersituational stability of the four temporal characteristics. Sufficient intersituational stability is another prerequisite for deep-level team psychological inputs to provide valid and consistent predictions on team processes and team outputs. If a psychological attribute is found to vary across situations and subject to the influence of situational factors, it would be difficult for researchers to conclude whether team members' attribute leads to certain team processes and/or team outputs or, vice versa, they are determined by certain team processes and/or team outputs. In addition, the attribute's predictions may be contingent on research scenarios and result in inconsistent predictions across studies. Hence, the aim of the present study is to examine the intersituational stability of the four temporal characteristics as a preliminary step before studying their power to predict team processes and team outputs.

\section{Theoretical Background}

The notion of intersituational stability refers to the absence of differences in subjects' attribute levels across situations. Although situations can differ in many aspects, we focus on those that differ in their influence on individuals' temporal experience and distinguish between "strong" and "weak" situations accordingly. House, Shane, and Herold (1996) define a strong situation as a situation that provides unambiguous cues as to appropriate behaviors of individuals. A strong situation will drive individuals' attitude, behavior, or trait to the particular kind or level required by the situation. It will create greater homogeneity of individuals' attitude, behavior, or trait than a weak situation does, and make subjects' attribute levels converge to a larger degree than a weak situation. In this study, we seek to create a situation that contains strong temporal cues and compare it to a situation that contains weak temporal cues. The designed strong situation is supposed to influence individuals' time urgency, polychronicity, time perspective, and temporal depth, whereas the naturalistic weak situation is supposed not to exert any particular influence on these temporal characteristics of individuals.

The assessment of the intersituational stability of time urgency, polychronicity, time perspective, and temporal depth requires at least two measurement moments. Accordingly, the research design requires one group of subjects to be exposed to a weak situation and a strong situation subsequently and another group of 
subjects to two weak situations subsequently. A comparison of subjects' attribute level change over time between the two conditions will help to identify whether subjects' attribute level change can be attributed to the change of situations or merely to the flow of time. If a psychological attribute is stable across situations, the comparison will demonstrate similar intertemporal stability between the two conditions; if the attribute is influenced by the situation, the intertemporal stability will be lower in the weak-strong condition than in the weak-weak condition, and subjects' attribute levels in the weak-strong condition will change to the direction indicated by the strong situation. Given that the intersituational stability test involves the intertemporal stability test, an equivalent measurement instrument is a prerequisite for testing attribute level change over time and across situations. Change in subjects' rank orders of the attribute levels over time and change in their attribute levels over time should also be tested separately.

To our knowledge, the extant organizational behavior literature has hardly addressed the intersituational stability of the four temporal characteristics. In the coming sections, rather than repeating the same literature review as in Chapter 2, we present the research design, analyses, and results regarding intersituational stability, and discuss the implications of this study for future team research and management practice.

\section{Methods}

In a quasi-experiment, we tested the stability of time urgency, polychronicity, time perspective, and temporal depth, over a period of one month in the two conditions. We created an experimental condition consisting of a weak naturalistic situation and a strong simulated situation and a control condition consisting of two weak situations. The strong situation was created by a computer simulation that aimed to increase subjects' time urgency and present time perspective, and decrease their polychronicity, past time perspective, future time perspective, and temporal depth. For each dimension of time urgency, polychronicity, and time perspective, we progressively tested whether the intertemporal stability of construct validity, construct calibration, and construct level are comparable in the two conditions. We examined the intersituational stability (or equivalence) of the attribute level, if and only if the measurement instrument was found to be equivalent over time and between the two conditions (in terms of construct validity and calibration). The definition of strong situation suggests the convergence of individuals' attitude, behavior, or trait to a particular type or level. We therefore conclude that there is a situational effect on a temporal characteristic, if (1) the within-group variance of an attribute level decreases over time to a larger extent in the experimental condition than in the control condition and/or (2) over time, the group mean of time urgency and present time perspective increases more or decreases less in the experimental condition than in 
the control condition, and the group mean of polychronicity, past time perspective, future time perspective, and temporal depth decreases more or increases less in the experimental condition than in the control condition.

\section{Design}

In the quasi-experiment, we formed an experimental group and a control group (referred to as $\mathrm{G}_{1}$ and $\mathrm{G}_{0}$ respectively in the coming sections), and repeatedly measured time urgency, polychronicity, time perspective, and temporal depth three times over two months (referred to as $T_{1}, T_{2}$, and $T_{3}$ in the following sections). The only between-group difference in the research design was that in the experimental group, we asked the participants to fill in the $T_{2}$ questionnaire immediately after they completed a computer simulation of corporate crisis management. In the role-play game, we asked participants to manage a series of crises as the Chief Executive Officer (CEO) of a Seattle-based consumer electronics company. With the simulation, we aimed to intensify participants' time urgent feelings and direct their attentions and efforts to this particular here-and-now assignment. Participants' timeurgent feelings were expected to be enhanced, since they were asked to complete the simulation with the most correct decisions and as fast as possible in order to obtain cash prize and avoid failing the course session ${ }^{18}$. Their present time perspective was expected to be enhanced and their polychronicity, past and future time perspective, and temporal depth to be lessened by the here-and-now assignment.

\section{Simulation}

The simulated crises start from a fire incident in Seattle of the United States of America. The city's fire department suspects that the fire is caused by an electronic coffee maker from Martha@Home Co., one of the company's most popular models. After the coverage of the incident by a local newspaper, the company's product safety and overall reputation is in question by the public. Meanwhile, an anonymous employee of the company goes to a local television channel, suggesting that the fire incident may be related to the misconducts of the company's procurement manager. In the end, the fire department and police department find that the fire is caused by an old electricity cable connected to the fridge in the house but the procurement manager does commit corruptions by receiving illegitimate commissions from one of the company's suppliers.

The simulation consists of 10 critical stages. In each stage, a participant, playing the role of the company's CEO, must decide for the best reaction in the particular situation. Information about the latest development of the crises is introduced before each decision-making moment by means of videos, audios, or text descriptions. Decisions to be made include, for example, what information to be released to

\footnotetext{
${ }^{18}$ The "punishment" of failing the course session was not real and participants were debriefed immediately after the simulation ended.
} 
the public, what specific audience (e.g. customers, stockholders, employees, suppliers) to be addressed, whether to launch a nationwide product recall, and whether to fire the procurement manager suspected of corruption charges. We developed the simulation based on real-life corporate crisis management cases (e.g. the Black \& Decker coffee maker recall in 1988, Smith, Thomas, \& Quelch, 1996), ethical issues in companies' procurement (Donaldson, 1996; Rudelius, \& Buchholz, 1979), and crisis management theories (Eccles, Newquist, \& Schatz, 2007; Kellerman, 2006; Pearson \& Clair, 1998). We present the description of the 10 critical stages of the simulation Appendix C.

\section{Procedure}

Students from the Faculty of Economics and Business Administration ${ }^{19}$ of Maastricht University participated in our study. Students who attended the Crisis Management course, a third-year bachelor course, in 2007 were assigned to the experimental group. With the support of the course coordinator, we designed the computer simulation into the course curriculum as an obligatory assignment in the midterm. Students who attended the International Business Strategy course, a secondyear bachelor course, in 2008 were assigned to the control group. No students had ever participated in both courses. Time urgency, polychronicity, time perspective, and temporal depth were repeatedly measured in Week 1 (i.e. $T_{1}$ ), Week 4 (i.e. $T_{2}$ ), and Week 7 (i.e. $T_{3}$ ) in each course.

The simulation took place in two computer laboratories in two sessions. We asked students who attended the morning (afternoon) tutorials to participate in the first (second) session and randomly assigned them to each laboratory. The course reader stated that students' performance in the simulation was counted as part of their participation grades. Before each simulation started, the course coordinator and I informed that a person's simulation performance depended on how many "correct" decisions the person made in the simulation and how fast the person completed the simulation. We also announced a false "reward-punishment" policy that the person who made most correct decisions and completed the simulation in the shortest time would be rewarded with a 50 -Euro cash prize ${ }^{20}$ and that a person needed at least six correct answers in order to pass the simulation and would fail the participation of the session. Immediately after all the participants completed the simulation and the attached $\mathrm{T}_{2}$ questionnaire, we debriefed that the cash prize was for real but not the "punishment" for failing the simulation. The final part of the simulation was to fill in the $\mathrm{T}_{2}$ questionnaire.

\footnotetext{
${ }^{19}$ See Footnote No.12 (p.28).

${ }^{20}$ See Footnote No.14 (p.28).
} 


\section{Sample}

Our original plan was to examine and compare the intertemporal stability of time urgency, polychronicity, time perspective, and temporal depth between the experimental and control group with the data from three time moments. However, high participant dropout rate and consequent small sample size of complete cases made it impossible to use the three-moment data. In the experimental group, 63 students completed the $T_{1}$ questionnaire; 52 the simulation and $T_{2}$ questionnaire; 20 the $T_{3}$ questionnaire. In the control group, 124 students completed the T1 questionnaire; 49 the $T_{2}$ questionnaire; 34 the $T_{3}$ questionnaire. Small sample sizes tend to generate unreliable model estimations; therefore, we used only the $T_{1}$ and $T_{2}$ data for further intersituational stability test. In the end, the experimental group contained 52 complete cases; the control group contained 49 complete cases.

\section{Measures}

Time urgency. Time urgency was measured by the same $17^{21}$ five-Likert point items selected from the 33-item scale in Landy, Rastegary, Thayer, \& Colvin (1991) study as in Chapter 2. We selected the items by the two aforementioned criteria (p.36). First, each selected item must load on a unique construct with an above- 0.50 factor loading in the original study. Second, at least three items are present for each construct. These selection criteria ensure the inclusion of the most robust items for a subconstruct and consider the tradeoff between questionnaire length and participants' continuous participation in the longitudinal study. As a result, eating behavior include such items as "my spouse and close friends told me that I eat too fast;" competitiveness "I am competitive and hard-driving;" general hurry "I usually feel much pressured by time;" and task-related hurry "I usually work fast and energetically." We asked each participant how well each item described him or her from the choices provided in the scale. The measurement of time urgency and other included temporal characteristics and situational strength is presented in Appendix A (Scale $1,3,4,6,7)$.

Polychronicity. In the experimental group, polychronicity was measured by six five-Likert-point items selected from Bluedorn, Kalliath, Strube, and Martin's (1998) Inventory of Polychronic Attitudes Inventory (IPV). We adapted the collective-level measure to an individual-level measure by replacing "we" with "I" in the items. We excluded one item that is negatively phrased (i.e. "I seldom like ...") and three items that connote clear preferences and are phrased similarly to other items (e.g. "I would rather . .."). The included items are, for example, "I like to juggle several activities at the same time," "I believe people should try to do many things at once," and "I believe it is best to complete one task before beginning another (R)." In the control group, polychronicity was measured by the four-item five-

\footnotetext{
${ }^{21}$ Two items of competitiveness were excluded from the stability test, due to the small sample size $\left(\mathrm{n}_{0}=49, \mathrm{n}_{1}=52\right)$.
} 
Likert-point Polychronic Attitudes Inventory (PAI, Bluedorn, Kaufman, \& Lane, 1992), including such items as "I do not like juggle several activities at the same time (R)," "People should not try to do many things at once (R)," "When I sit down at my desk, I work on one project at a time (R)," and "I am comfortable doing several things at the same time." The letter $\mathrm{R}$ in the brackets indicates reverse wording. We asked each respondent to what degree he or she agreed with each item.

Time perspective. Time perspective was measured $22^{22}$ five-point Likert items selected from the 56-item Zimbardo Time Perspective Inventory (Zimbardo \& Boyd, 1999) by the two aforementioned criteria. The selected items measured all the five subconstructs, including past-positive, past-negative, present-hedonistic, present-fatalistic, and future-orientation. The included items are, for example, "familiar childhood signs, sounds, smells often bring back a flood of wonderful memories," "it is hard for me to forget unpleasant images of my youth," "It is important to put excitement in my life," "my life path is controlled by forces I cannot influence," and "meeting tomorrow's deadlines and doing other necessary work comes before tonight's play." We asked each respondent how well each item described him or her.

Temporal depth. We measured temporal depth by asking each respondent to specify how much exact time he or she refers to "short term future," "mid-term future," "long-term future," "recently," "a middling time ago," and "a long time ago" (Bluedorn, 2002). Fifteen choices were offered for each phrase, including one day, one week, two weeks, one month, three months, six months, nine months, one year, three years, five years, ten years, fifteen years, twenty years, twenty-five years, and more than twenty-five years.

Additional measures. We added four items that measured situational strength at the end of the $\mathrm{T}_{2}$ questionnaire in the experimental group. These items examined how seriously a participant took the simulation. We asked on a five-point Likert scale to what degree a person "wanted achieve the best performance during the simulation," "wanted to finish the simulation as quickly as possible," "was worried about what results I would get from the simulation" and "felt anxious and nervous during the simulation." The group mean for the four items were $4,3.25,3.16$, and 2.27 , respectively.

\section{Analyses}

In the preliminary analyses, we examined the randomness of missing data in the experimental and control group respectively. We compared the $T_{1}$ score differences of each temporal characteristic between the all-time committed respondents and dropout respondents in each group. A significant differences implies that the missing data was associated with a temporal characteristic and the sample distribution

\footnotetext{
${ }^{22}$ One item of past-positive was excluded in the stability test, due to the small sample size $\left(\mathrm{n}_{0}=49\right.$, $\left.\mathrm{n}_{1}=52\right)$.
} 
did not represent the population distribution of that characteristic. We then eliminated the temporal characteristic from further stability test.

For each dimension of time urgency and time perspective, and for polychronicity, we tested the intertemporal stability between the experimental and control group via the alpha-beta-gamma-change typology (Golembiewski, Billingsley, \& Yeager, 1976) and LGM procedure (Chan, 1998; Lance, Vandenberg, \& Self, 2000). With six progressively restricted models (M1 to M6) and an additional model (M7), we tested whether the factor structure (M1), factor loadings (M2), item intercepts (M3), item error terms (M4), factor variance (M5), and factor mean (M7), were equal between $T_{1}$ and $T_{2}$ and between the two groups and whether the factor covariance between $T_{1}$ and $T_{2}$ (M6) was equal in the two groups. Model fit was assessed by Chi-square, comparative fit index (CFI), and root mean squared error of approximation (RMSEA). An above $.95 \mathrm{CFI}$ and a below .05 RMSEA (Bollen, 1989) indicate a good model fit. We present the LISREL syntax with eating behavior as an example in Appendix B

The simulated strong situation was to increase time urgency and present time perspective and to decrease polychronicity, past time perspective, future time perspective, and temporal depth. We expected that (1) construct measurement was equivalent over time in each group, and (2) the factor variance of each construct changed over time to a larger degree in the experimental group than in the control group, and/or (3) over time, the factor mean of each time urgency dimension, and the present-hedonistic and present-fatalistic orientations of time perspective increased to a larger degree or decreased to a lesser degree in the experimental group than in the control group, and, over time, the factor mean of polychronicity and past-positive, past-negative, and future-orientation of time perspective decreased to a larger degree or increased to a lesser degree in the experimental group than in the control group.

Furthermore, we performed a repeated-measures Multivariate Analysis of Variances (MANOVA) to test whether the intertemporal stability of temporal depth differed between the experimental and control group, since, unlike the other temporal characteristics, each aspect of temporal depth (i.e. short-, mid-, and long-term past temporal depth, and short-, mid-, and long-term future temporal depth) was measured by one questionnaire item.

\section{Results}

The preliminary analysis results suggest that in each group, data missing was random and unrelated to any temporal characteristic. We present in Table 3.1 to Table 3.4 the LGM analysis results for time urgency, polychronicity, and time perspective and repeated-measures MANOVA results for temporal depth between the two groups. In Table 3.5, we summarize the between-group comparisons. 
Table 3.1 The LGM Analysis of the Intersituational Stability of Time Urgency between the Control and Experimental Group $\left(\mathrm{n}_{0}=49, \mathrm{n}_{1}=52\right)$

\begin{tabular}{lcccccccc}
\hline \multicolumn{1}{c}{ Model } & \multicolumn{4}{c}{ Eating behaviors } & \multicolumn{5}{c}{ Competitiveness } \\
\cline { 2 - 9 } & df & $\chi^{2}$ & RMSEA & CFI & df & $\chi^{2}$ & RMSEA & CFI \\
\hline 1. Equivalent factor & 94 & 106.21 & .05 & .99 & 59 & 86.87 & .10 & .90 \\
structure & 8 & 2.2 & -- & -- & 6 & 0.35 & -- & -- \\
1 vs. 2 & 102 & 108.41 & .04 & .99 & 65 & 87.22 & .08 & .92 \\
$\begin{array}{l}\text { 2. Equal factor loa- } \\
\text { dings }\end{array}$ & 10 & 5.63 & -- & -- & 8 & 3.5 & -- & -- \\
2 vs. 3 & 112 & 114.04 & .02 & .99 & 73 & 90.72 & .07 & .93 \\
3. Equal item inter- \\
cepts & 5 & 6.6 & -- & -- & 4 & 3.26 & -- & -- \\
3 vs.4 & 117 & 120.64 & .03 & .99 & 77 & 93.98 & .07 & .93 \\
$\begin{array}{l}\text { 4. Equal error vari- } \\
\text { ances }\end{array}$ & 1 & 0.2 & -- & -- & 1 & 1.15 & -- & -- \\
4 vs.5 & 118 & 120.84 & .02 & .99 & 78 & 95.13 & .07 & .93 \\
5. Equal factor vari- \\
ances
\end{tabular}


Table 3.1 (continued) $\left(\mathrm{n}_{0}=49, \mathrm{n}_{1}=52\right)$

\begin{tabular}{|c|c|c|c|c|c|c|c|c|}
\hline \multirow[t]{2}{*}{ Model } & \multicolumn{4}{|c|}{ General hurry } & \multicolumn{4}{|c|}{ Task-Related hurry } \\
\hline & $\overline{d f}$ & $\chi^{2}$ & RMSEA & CFI & $\mathrm{df}$ & $\chi^{2}$ & RMSEA & CFI \\
\hline $\begin{array}{l}\text { 1. Equivalent } \\
\text { factor structure }\end{array}$ & 32 & 30.20 & .00 & .99 & 32 & 37.15 & .06 & .97 \\
\hline 1 vs. 2 & 4 & 1.93 & -- & -- & 4 & $10.66^{* *}$ & -- & -- \\
\hline $\begin{array}{l}\text { 2. Equal factor } \\
\text { loadings }\end{array}$ & 36 & 32.13 & .00 & 1.00 & 36 & 47.81 & .08 & .96 \\
\hline 2 vs. 3 & 6 & $12.71 * *$ & -- & -- & 6 & 1.47 & -- & -- \\
\hline $\begin{array}{l}\text { 3. Equal item } \\
\text { intercepts }\end{array}$ & 42 & 44.84 & .04 & .99 & 42 & 49.28 & .06 & .98 \\
\hline 3 vs. 4 & 3 & 2.23 & -- & -- & 3 & -0.05 & -- & -- \\
\hline $\begin{array}{l}\text { 4. Equal error } \\
\text { variances }\end{array}$ & 45 & 47.07 & .03 & .99 & 45 & 49.23 & .04 & .98 \\
\hline 4 vs. 5 & 1 & 1.2 & -- & -- & 1 & 0.05 & -- & -- \\
\hline $\begin{array}{l}\text { 5. Equal factor } \\
\text { variances }\end{array}$ & 46 & 48.27 & .03 & .99 & 46 & 49.28 & .04 & .99 \\
\hline 5 vs. 6 & 1 & 0 & -- & -- & 1 & -0.20 & -- & -- \\
\hline $\begin{array}{l}\text { 6. Equal factor } \\
\text { covariances }\end{array}$ & 47 & 48.27 & .03 & .99 & 47 & 49.08 & .03 & .99 \\
\hline 6 vs. 7 & 0 & 1.15 & -- & -- & 0 & 0.46 & -- & -- \\
\hline $\begin{array}{l}\text { 7. Equal factor } \\
\text { means }\end{array}$ & 47 & 49.42 & .03 & .99 & 47 & 49.54 & .03 & .99 \\
\hline
\end{tabular}


Table 3.2 The LGM Analysis of the Intersituational Stability of Polychronicity between the Control and Experimental Group $\left(\mathrm{n}_{0}=49, \mathrm{n}_{1}=52\right)$

\begin{tabular}{lcccc}
\hline \multirow{2}{*}{ Model } & \multicolumn{4}{c}{ Polychronicity } \\
\cline { 2 - 5 } & $\mathrm{df}$ & $\chi^{2}$ & RMSEA & CFI \\
\hline 1. Equivalent factor structure & 59 & 132.48 & .16 & .78 \\
1 vs. 2 & 6 & 2.37 & -- & -- \\
2. Equal factor loadings & 65 & 134.85 & .15 & .79 \\
2 vs. 3 & 8 & $26.06^{* * *}$ & -- & -- \\
3. Equal item intercepts & 73 & 160.91 & .16 & .76 \\
3 vs.4 & 4 & 7.5 & -- & -- \\
4. Equal error variances & 77 & 168.41 & .15 & .77 \\
4 vs.5 & 1 & 1.3 & -- & -- \\
5. Equal factor variances & 78 & 169.71 & .15 & .77 \\
5 vs. 6 & 1 & -0.02 & -- & -- \\
6. Equal factor covariances & 79 & 169.69 & .15 & .77 \\
6 vs.7 & 0 & 3.71 & -- & -- \\
7. Equal factor means & 79 & 173.40 & .16 & .76 \\
\hline
\end{tabular}


Table 3.3 The LGM Analysis of the Intersituational Stability of Time Perspective between the Control and Experimental Group $\left(\mathrm{n}_{0}=49, \mathrm{n}_{1}=52\right)$

\begin{tabular}{llllllllll}
\hline Model & \multicolumn{4}{c}{ Past-Positive } & \multicolumn{5}{c}{ Past-Negative } \\
\cline { 2 - 9 } & df & $\chi^{2}$ & RMSEA & CFI & df & $\chi^{2}$ & RMSEA & CFI \\
\hline 1. Equivalent & 94 & 110.47 & .06 & .92 & 94 & 124.22 & .08 & .95 \\
factor structure & 8 & 3.85 & -- & -- & 8 & 3.72 & -- & -- \\
$\begin{array}{l}\text { 1 vs. 2 } \\
\text { 2. Equal factor }\end{array}$ & 102 & 114.32 & .05 & .93 & 102 & 127.94 & .07 & .95 \\
loadings & 10 & 10.98 & -- & -- & 10 & 6.68 & -- & -- \\
$\begin{array}{l}\text { 2 vs. } 3 \\
\text { 3. Equal item }\end{array}$ & 112 & 125.30 & .05 & .92 & 112 & 134.62 & .06 & .96 \\
intercepts & 5 & 5.21 & -- & -- & 5 & 8.01 & -- & -- \\
$\begin{array}{l}\text { 3 vs.4 } \\
\text { 4. Equal error }\end{array}$ & 117 & 130.51 & .05 & .92 & 117 & 142.63 & .07 & .95 \\
variances & 1 & 0.02 & -- & -- & 1 & 0.46 & -- & -- \\
$\begin{array}{l}\text { 4 vs.5 } \\
\text { 5. Equal factor }\end{array}$ & 118 & 130.53 & .05 & .93 & 118 & 143.09 & .07 & .95 \\
variances & 1 & -0.12 & -- & -- & 1 & 0.01 & -- & -- \\
$\begin{array}{l}\text { 5 vs. 6 } \\
\text { 6. Equal factor }\end{array}$ & 119 & 130.41 & .04 & .93 & 119 & 143.10 & .06 & .95 \\
covariances & 0 & 0.09 & -- & -- & 0 & 10.05 & -- & -- \\
$\begin{array}{l}\text { 6 vs.7 } \\
\text { 7. Equal factor } \\
\text { means }\end{array}$ & 119 & 130.50 & .04 & .93 & 119 & 153.15 & .08 & .94 \\
\hline
\end{tabular}


Table 3.3 (Continued.)

\begin{tabular}{|c|c|c|c|c|c|c|c|c|}
\hline \multirow[t]{2}{*}{ Model } & \multicolumn{4}{|c|}{ Present-Hedonistic } & \multicolumn{4}{|c|}{ Present-Fatalistic } \\
\hline & $\mathrm{df}$ & $\chi^{2}$ & RMSEA & CFI & df & $\chi^{2}$ & RMSEA & CFI \\
\hline $\begin{array}{l}\text { 1. Equivalent factor } \\
\text { structure }\end{array}$ & 32 & 42.84 & .08 & .95 & 59 & 71.73 & .07 & .93 \\
\hline 1 vs. 2 & 4 & 0.27 & -- & -- & 6 & 1.77 & -- & -- \\
\hline $\begin{array}{l}\text { 2. Equal factor loa- } \\
\text { dings }\end{array}$ & 36 & 43.11 & .06 & .96 & 65 & 73.50 & .05 & .94 \\
\hline 2 vs. 3 & 6 & 6.72 & -- & -- & 8 & 2.42 & -- & -- \\
\hline $\begin{array}{l}\text { 3. Equal item inter- } \\
\text { cepts }\end{array}$ & 42 & 49.83 & .06 & .96 & 73 & 75.92 & .03 & .96 \\
\hline 3 vs. 4 & 3 & 0.38 & -- & -- & 4 & -0.25 & -- & -- \\
\hline $\begin{array}{l}\text { 4. Equal error varian- } \\
\text { ces }\end{array}$ & 45 & 50.21 & .05 & .97 & 77 & 75.67 & .00 & .97 \\
\hline 4 vs.5 & 1 & $5.57 * *$ & -- & -- & 1 & 4.93 & -- & -- \\
\hline $\begin{array}{l}\text { 5. Equal factor vari- } \\
\text { ances }\end{array}$ & 46 & 55.78 & .07 & .96 & 78 & 80.60 & .03 & .95 \\
\hline 5 vs. 6 & 1 & 0 & -- & -- & 1 & 0.04 & -- & -- \\
\hline $\begin{array}{l}\text { 6. Equal factor cova- } \\
\text { riances }\end{array}$ & 47 & 55.78 & .06 & .96 & 79 & 80.64 & .02 & .96 \\
\hline 6 vs. 7 & 0 & -1.07 & -- & -- & 0 & 0.34 & -- & -- \\
\hline 7. Equal factor means & 47 & 54.71 & .06 & .96 & 79 & 80.98 & .02 & .95 \\
\hline
\end{tabular}


Table 3.3 (Continued.)

\begin{tabular}{lcccc}
\hline \multirow{1}{*}{ Model } & \multicolumn{4}{c}{ Future-Oriented } \\
\cline { 2 - 5 } & $\mathrm{df}$ & $\chi^{2}$ & RMSEA & CFI \\
\hline 1. Equivalent factor structure & 59 & 71.16 & .07 & .97 \\
1 vs. 2 & 6 & 6.68 & -- & -- \\
2. Equal factor loadings & 65 & 77.84 & .06 & .98 \\
2 vs. 3 & 8 & 3.17 & -- & -- \\
3. Equal item intercepts & 73 & 81.01 & .05 & .99 \\
3 vs.4 & 4 & 0.85 & -- & -- \\
4. Equal error variances & 77 & 81.86 & .04 & .99 \\
4 vs.5 & 1 & 0.87 & -- & -- \\
5. Equal factor variances & 78 & 82.73 & .04 & .99 \\
5 vs. 6 & 1 & 0.01 & -- & -- \\
6. Equal factor covariances & 79 & 82.74 & .03 & 1.00 \\
6 vs.7 & 0 & 0.76 & -- & -- \\
7. Equal factor means & 79 & 83.50 & .03 & 1.00 \\
\hline
\end{tabular}




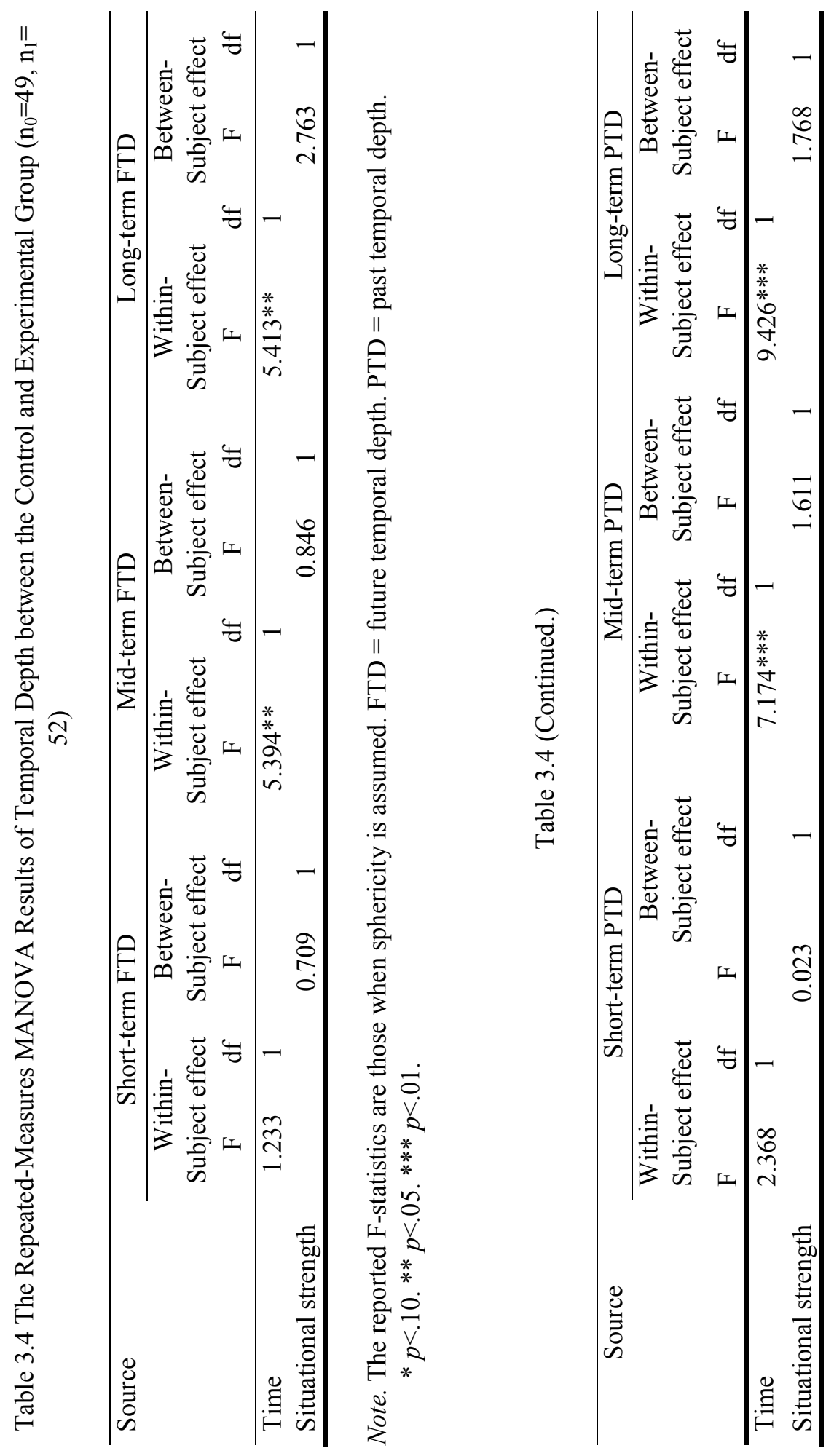


Table 3.5 Summary of Test Results for Time Urgency, Polychronicity, Time Perspective, and Temporal Depth in the Control and Experimental Group $\left(\mathrm{n}_{0}=49, \mathrm{n}_{1}=\right.$ 52)

\begin{tabular}{lccc}
\hline & \multicolumn{3}{c}{ Between-group comparison } \\
& $\gamma$ change & $\beta$ change & $\alpha$ change \\
\hline Time urgency & -- & -- & -- \\
Eating behaviors & $\checkmark$ & -- & -- \\
Competitiveness & -- & -- & -- \\
General hurry & $\checkmark$ & -- & -- \\
Task-related hurry & $\checkmark$ & -- & -- \\
Polychronicity & & & \\
Time perspective & $\checkmark$ & -- & -- \\
Past-Positive & $\checkmark$ & -- & -- \\
Past-Negative & $\checkmark$ & -- & -- \\
Present-Hedonistic & $\checkmark$ & -- & - \\
Present-Fatalistic & $\checkmark$ & -- & N.A. \\
Future-Orientation & N.A. & N.A. & \\
Temporal Depth & & & \\
\hline
\end{tabular}


For time urgency, the intertemporal stability of the factor structure differed between the two groups for competitiveness (CP) and task-related hurry (TRH), since M1 was rejected $\left(\mathrm{CFI}_{\mathrm{CP}}=.90, \mathrm{RMSEA}_{\mathrm{CP}}=.10 ; \mathrm{CFI}_{\mathrm{TRH}}=.97, \mathrm{RMSEA}_{\mathrm{TRH}}=.06\right)$. In additional analyses, we tested the intertemporal stability of the factor structure within the experimental group (G1) and control group (G0) respectively. We found that for competitiveness, the factor structure changed in both groups, since M1 was rejected $\left(\mathrm{CFI}_{\mathrm{CP}, \mathrm{Gl}}=.86, \mathrm{RMSEA}_{\mathrm{CP}, \mathrm{G} 1}=.14 ; \mathrm{CFI}_{\mathrm{CP}, \mathrm{G} 0}=.92, \mathrm{RMSEA}_{\mathrm{CP}, \mathrm{G} 0}=.12\right)$. For task-related hurry, the factor structure remained the same in both groups $\left(\mathrm{CFI}_{\mathrm{TRH}, \mathrm{Gl}}=1.00, \mathrm{RMSEA}_{\mathrm{TRH}, \mathrm{Gl}}=.00 ; \mathrm{CFI}_{\mathrm{TRH}, \mathrm{G} 0}=1.00, \mathrm{RMSEA}_{\mathrm{TRH}, \mathrm{G} 0}=.00\right)$, suggesting that the change in factor structure was due to situational strength but not to the flow of time. For eating behavior and general hurry, the attribute level remained stable between the two groups, since M1 to M7 were all accepted. It suggests that eating behavior and general hurry were rather stable over a short time period (i.e. one month) and across situations.

For polychronicity (PC), the intertemporal stability of the factor structure differed between the two groups, since M1 was rejected $\left(\mathrm{CFI}=.78, \mathrm{RMSEA}_{\mathrm{CP}}=.16\right)$. In additional analyses, we tested the intertemporal stability of the factor structure within the experimental group (G1) and control group (G0) respectively. We found that the factor changed between in both groups, since $\mathrm{M} 1$ was rejected $\left(\mathrm{CFI}_{\mathrm{G} 1}=.87\right.$, RMSEA $_{\mathrm{G} 1}=.17 ; \mathrm{CFI}_{\mathrm{G} 0}=.95, \mathrm{RMSEA}_{\mathrm{G} 0}=.09$ ).

For time perspective, the intertemporal stability of the factor structure differed for all the dimensions, including past-positive (PP), past-negative (PN), presenthedonistic $(\mathrm{PH})$, present-fatalistic $(\mathrm{PF})$, and future-oriented (FO), since M1 was rejected $\left(\mathrm{CFI}_{\mathrm{PP}}=.92, \quad \mathrm{RMSEA}_{\mathrm{PP}}=.06 ; \mathrm{CFI}_{\mathrm{PN}}=.95, \mathrm{RMSEA}_{\mathrm{PN}}=.08 ; \mathrm{CFI}_{\mathrm{PH}}=.95\right.$, $\left.\mathrm{RMSEA}_{\mathrm{PH}}=.08 ; \mathrm{CFI}_{\mathrm{PF}}=.93, \mathrm{RMSEA}_{\mathrm{PF}}=.07 ; \mathrm{CFI}_{\mathrm{FO}}=.97, \mathrm{RMSEA}_{\mathrm{FO}}=.07\right)$. In additional analyses, we tested the intertemporal stability of the factor structure in the experimental group (G1) and control group (G0) respectively. We found that for past-negative the factor structure changed in both groups, since M1 was rejected $\left(\mathrm{CFI}_{\mathrm{PN}, \mathrm{G} 1}=.93\right.$, $\mathrm{RMSEA}_{\mathrm{PN}, \mathrm{G} 1}=.13 ; \mathrm{CFI}_{\mathrm{PN}, \mathrm{G} 0}=.98$, $\left.\mathrm{RMSEA}_{\mathrm{PN}, \mathrm{G} 0}=.07\right)$. For pastpositive and present-fatalistic the factor structure changed only in the control group but not in the experimental group $\left(\mathrm{CFI}_{\mathrm{PP}, \mathrm{G} 1}=1.00, \mathrm{RMSEA}_{\mathrm{PP}, \mathrm{G} 1}=.00 ; \mathrm{CFI}_{\mathrm{PP}, \mathrm{G} 0}=.84\right.$, RMSEA $_{\mathrm{PP}, \mathrm{G} 0}=.12 ; \quad \mathrm{CFI}_{\mathrm{PF}, \mathrm{G} 1}=.98, \quad \mathrm{RMSEA}_{\mathrm{PF}, \mathrm{G} 1}=.05 ; \quad \mathrm{CFI}_{\mathrm{PF}, \mathrm{G} 0}=.94$, $\left.\mathrm{RMSEA}_{\mathrm{PF}, \mathrm{G} 0}=.07\right)$. For present-hedonistic and future-oriented the factor structure remained the same in both groups $\left(\mathrm{CFI}_{\mathrm{PH}, \mathrm{Gl}}=1.00\right.$, $\mathrm{RMSEA}_{\mathrm{PH}, \mathrm{Gl}}=.01$; $\mathrm{CFI}_{\mathrm{PH}, \mathrm{G} 0}=1.00, \mathrm{RMSEA}_{\mathrm{PH}, \mathrm{G} 0}=.00 ; \mathrm{CFI}_{\mathrm{FO}, \mathrm{G} 1}=1.00, \mathrm{RMSEA}_{\mathrm{FO}, \mathrm{G} 1}=.00 ; \mathrm{CFI}_{\mathrm{FO}, \mathrm{G} 0}=.99$, $\mathrm{RMSEA}_{\mathrm{FO}, \mathrm{G} 0}=.02$ ), suggesting that the observed change in factor structure was due to situational strength but not to the flow of time.

For temporal depth the repeated-measures MANOVA results show no between-group difference in the intertemporal stability of any facet of temporal depth, which suggests high intersituational stability for temporal depth. Within-subject effects, however, show a significant change in midterm future temporal depth $\left(\mathrm{F}_{(1,}\right.$, $\left.{ }_{101)}=5.394, p<.05\right)$, long-term future temporal depth $\left(\mathrm{F}_{(1,101)}=5.413, p<.05\right)$, midterm past temporal depth $\left.\mathrm{F}_{(1,101)}=7.174, p<.01\right)$, and long-term past temporal depth 
$\left(\mathrm{F}_{(1,101)}=9.426, p<.01\right)$ in both groups. We then performed additional repeatedmeasures MANOVA in each group and found that all facets of temporal depth significantly decreased between $T_{1}$ and $T_{2}$ in at least one group. Midterm future temporal depth, long-term future temporal depth, and midterm past temporal depth were rather stable in the experimental group, but dropped from 7.83 months to 6.42 months $\left(\mathrm{F}_{(1,49)}=2.876, p<0.10\right) 5.88$ years to 3.69 years $\left(\mathrm{F}_{(1,49)}=3.774, p<0.1\right)$, and six months to 4.16 months $\left(\mathrm{F}_{(1,49)}=5.020, p<0.5\right)$ in the control group respectively. Long-term past temporal depth dropped from 4.08 years to 2.65 in the experimental group $\left(\mathrm{F}_{(1,52)}=5.771, p<.05\right)$ and from 4.8 years to 3.57 years in the control group $\left(\mathrm{F}_{(1,49)}=3.806, p<.10\right)$.

\section{Discussion}

The aim of this study was to examine the intersituational stability of time urgency, polychronicity, time perspective, and temporal depth. Results from our longitudinal quasi-experiment suggest that most temporal characteristics are subject to situational influence. In particular, competitiveness and task-related hurry of time urgency, polychronicity, and past-positive, past-negative, present-hedonistic, presentfatalistic, and future-orientation of time perspective differ in construct validity between a condition consisting of a weak situation and a strong situation and a condition consisting two weak situations. Competitiveness and past-negative show a change in factor structure over time in both conditions; past-positive and presentfatalistic vary in factor structure over time in the weak-weak condition but not in the weak-strong condition. In these cases, the intertemporal stability of the factor structures cannot be compared between the two conditions, and hence not be attributed to the differences in situational strength. On the other hand, task-related hurry, present-hedonistic, and future-orientation vary in facture structure between the two conditions but not over time. In other words, the change in the constructs' factor structures can be attributed to the differences in situational strength but not to the flow of time. This means that temporal cues in specific situations can lead individuals to associate working speed, hedonistic view of the present, and being futureoriented with distinct sets of attitudes and behaviors. Moreover, eating behavior and general hurry of time urgency are rather stable across situations, given the high stability of measurement and attribute level between the weak-strong condition and the weak-weak condition.

For temporal depth, the difference in situational strength does not lead to different change patterns. Midterm and long-term future temporal depth and midterm past temporal depth decreases over time in the naturalistic weak-weak condition and long-term past temporal depth decreases in both the naturalistic weak-weak condition and the simulated weak-strong condition.

It is worth noting that the results from this chapter and Chapter 2 seem to differ as for the intertemporal stability of certain temporal characteristics under the 
naturalistic condition. For example, eating behavior and general hurry of time urgency are found to be stable in this chapter but were found to vary in measurement validity over time in Chapter 2. Two factors may explain such different findings. First, research interval differs between the two chapters (i.e. one month versus six months). As Zaheer, Albert, and Zaheer (1999) suggest, different observation intervals can lead researchers to discover different temporal dynamic patterns of the same research phenomenon. Second, the number of measurement moments also differs between the two chapters (i.e. two moments versus three moments). As Roe (2009) suggest, the number of measurement moments is very likely to produce different divergent and/or convergent temporal dynamics of individual cases.

\section{Theoretical Implications}

We draw three major implications from the study for future team research. First, time urgency, polychronicity, time perspective, and temporal depth are malleable across situations. Similar to low intertemporal stability, low intersituational stability of the four temporal characteristics raise the concerns of reverse causality and situation-contingent predictions when they are employed to predict time-related team processes (e.g. pacing, attention to time) and time-related team outputs (e.g. productivity by time, meeting project deadlines). It is difficult to conclude whether team members' time urgent feelings, multitasking preference, views about the past, present, or future, and cognitive framework into the past and future has an influence how teams pace their tasks over time and/or how well the teams perform temporally, or they are determined by the time-related team processes and team performance. Moreover, the four temporal characteristics may generate inconsistent predictions on the time-related team processes and team outputs, which are contingent on the temporal situations in which the temporal characteristics are measured. The argument can be extended to other psychological attributes that are used to define deep-level team team inputs such as personality traits. Researchers need to verify sufficient intersituational stability of such attributes before using them as predictors of team processes and outcomes.

Second, we suggest three criteria for testing the intersituational stability of a psychological attribute. A strong situation is, by definition, a situation that makes individuals' attitude, behavior, or trait to converge to a particular kind or level. The definition implies that the test of intersituational stability involves the test of intertemporal stability and, furthermore, that the intertemporal stability is lower in the group of subjects exposed to a weak-strong condition than those exposed to a weakweak situation. A situation effect exists when (1) the measurement instrument of an attribute is equivalent over time in both the weak-strong condition and weak-weak condition but may differ between the two condition, (2) the within-group variances have changed more drastically over time in the weak-strong situation than in the weak-weak situation, or (3) the group mean has changed more drastically over time in the weak-strong situation than in the weak-weak situation. Accordingly, the em- 
pirical testing should also contain the three steps. The alpha-beta-gamma-change typology (Golembiewski et al., 1976) and LGM procedure (Chan, 1998; Lance et al., 2000) provide a suitable methodology to test intersituational stability.

Finally, this study suggests that the separate dimensions of a multidimensional construct (e.g. time urgency, time perspective) can differ in their stability across situations, just as can be the case for intertemporal stability as Chapter 2 suggests. Researchers need to consider discriminant validity when empirically testing pertinent models. For example, researchers have theorized a positive impact of team members' time urgency on team meeting project deadlines (Waller, Conte, Gibson, $\&$ Carpenter, 2001). In empirical testing with a short research interval (e.g. one month), team members general feelings of time pressure (i.e. general hurry) may be a better predictor than their working speed (i.e. task-related hurry), because the former predictor is more resistant to situational influence than the latter predictor.

\section{Managerial Implications}

Given the methodological nature of the study, it is difficult to draw any direct implications for management practitioners. Yet, one might argue that that level of intersituational stability indicates the degree to which a temporal characteristic is subject to the influence of situational factors, and that certain situations can be purposely created or engineered to change individuals' temporal characteristic to the desirable level. For organizations, the reported low intersituational stability of most temporal characteristics indicate that organizations should be able to change individual employees' time-related attitudes and behaviors to a desirable level and cultivate desirable time-related organizational cultures (e.g. future-oriented culture, polychronic culture) by designed interventions such as time management training programs and strategically-set deadlines.

\section{Limitations}

There are three major limitations in this study. First, as in Chapter 2, the generalizability of the reported findings is limited - in this case to student samples and the research interval of one month. In both experimental and control group, we were unable to rule out the potential influence of the progression of the academic quarter on the levels of the four temporal characteristics. Replication of the study with other sample types and with time intervals of various lengths can help to obtain a comprehensive view of the intersituational stability of the four temporal characteristics. Second, the small sample size, that is, 52 participants in the experimental group and 49 in the control group, subjects the validity of the reported findings to question. Replication of the study with larger sample sizes is necessary. Third, the applied testing method (i.e. the alpha-beta-gamma-change typology and LGM procedure) examines the intertemporal (and intersituational) stability of attribute level at the group level but not at the individual level. When graphing individuals' development patterns of each construct of the four temporal characteristics, we may find that the 
group-level temporal dynamic patterns, estimated by the LGM and repeatedmeasures (M)ANOVA, do not apply to all the individuals. For example, in this study, we find that general hurry is stable over time at the group level in both the experimental and control condition. This issue will be examined in the following chapters. In Chapter 4, we elaborate upon the logical connection between grouplevel and individual-level temporal dynamics and propose a method to study temporal dynamics at the individual level. This method is applied in an empirical study in Chapter 5. 



\section{Chapter $4^{23}$}

\section{An Alternative Approach to Study Team Process Dynamics}

The conventional approach to analyze intra-subject change over time is growth analysis (for example, see Singer \& Willet, 2003). Among all, latent linear and quadratic growth modeling are among the most popular methods in the recent years. Higher-order models are less often used due to greater numbers of parameters to be estimated. These models are based on the assumption that individual-level growth trajectories are similar to the group-level trajectory. Individual subjects' growth coefficients (e.g. initial level and change rate) are considered to vary randomly across a sample and population. This approach to analyze intra-subject change over time poses a limitation, as it assumes "structural homogeneity" and treats information on individual idiosyncrasy as error, thereby precludes the discovery of "structural heterogeneity" at the individual level. In order to overcome this limitation, we develop an approach that acknowledges that subjects' growth trajectories differ from each other in a way that is not sufficiently captured by the varying growth parameters estimated in the linear or quadratic models. The new developed approach implies a bottom-up order (i.e. individuals-to-group) to examine change, in which individual trajectories are grouped on the basis of similarities. An advantage of such an approach is that it can identify non congruent growth trajectories and subsequently examine the antecedents and consequences of these trajectories, an approach we consider important for advancing team research that is based on the Input-Process-Output (IPO) framework.

In this chapter, we question the suitability of the conventional inter-subject longitudinal approach in team processes research, and particularly the "group-toindividuals" method of analysis. This method assumes homogeneous individual dynamic patterns of a psychological attribute (e.g. trait, attitude, behavior) over time and infers these patterns from an estimated group pattern. The inter-subject approach neither examines inter-subject (e.g. inter-person, inter-team) differences in attribute dynamic patterns over time, nor considers the nomological networks of intra-subject attribute change. Hence, we develop an intra-subject longitudinal ap-

\footnotetext{
${ }^{23}$ An updated version of this chapter is currently under review at a peer-reviewed journal (c.f. Li, J., \& Roe, R.A., Introducing an intra-subject longitudinal approach in the study of team process dynamics, Manuscript submitted for publication.)
} 
proach that allows for heterogeneity and regards intra-subject attribute dynamics over time as a temporally integrated concept. The new approach uses an "individuals-to-group" analysis method and infers the group's dynamic pattern from individual patterns. We begin the chapter by discussing the relationship between grouplevel and individual-level temporal dynamics. We elaborate the three-step intrasubject longitudinal approach, exemplify its application with temporal dynamic patterns defined by three measurement moments, and shortly discuss its merits and limitations. 
The empirical evidence presented in the previous chapters suggests that a psychological attribute that is stable over time at the group level can change drastically over time at the individual level. For instance, in Chapter 2, we found that the future-orientation component of time perspective is stable over time at the group level, but change drastically at the individual level (see Figure 2). These empirical observations suggest a logical asymmetry between the group-level and individuallevel temporal dynamics of a psychological attribute. On the one hand, the occurrence of group-level change implies the occurrence of individual-level change, although the degree of group-level change may not imply the same degree of individual-level change. Group-level attribute change can result from many individuals changing subtly, some individuals changing moderately, or a few individuals changing substantially in the attribute levels. On the other hand, the occurrence of individual-level change does not necessarily imply the occurrence of group-level change. When individuals' attribute development patterns diverge over time, consolidating heterogeneous individual patterns into one generic group pattern will result in a group pattern that does not represent what happens in the individual subjects in the group. Individual patterns of opposite development directions (i.e. increase versus decrease) may counterbalance each other at the group level and produce a stable group-level pattern. In the case of being stable, the logical asymmetry suggests that an attribute being stable at the individual level would imply it being stable at the group level, whereas the attribute being stable at the group level does not necessarily imply it being stable at the individual level. In sum, the relations between group-level and individual-level temporal dynamics are not symmetric. Group-level change is a sufficient but not necessary condition for individual-level change, whereas high group-level stability is a necessary but not sufficient condition for high individual-level stability.

Such logical asymmetry subjects to question the homogeneity assumption and group-to-individual analysis method of the inter-subject longitudinal approach that is conventionally used in extant longitudinal studies. The homogeneity assumption suggests that group-level temporal dynamic pattern is a simple consolidation, or aggregation, of homogenous individual patterns and, once known, automatically informs individual patterns. In the consequent analysis, the group-level pattern is decomposed into individual patterns that are considered to deviate from the grouplevel pattern in a random manner. In empirical investigations, the inter-subject approach can lead researchers to misinterpret high group-level intertemporal stability as lacking individual-level intra-subject change over time, an interpretation that may contradict the empirical reality. Therefore, the logical asymmetry prompts us to develop an alternative research approach that acknowledges the possibility of heterogeneous individual patterns (i.e. the heterogeneity assumption) and constructs group-level temporal dynamic pattern from the distribution of heterogeneous individual patterns (i.e. the individual-to-group construction method).

In the coming sections, we elaborate on the group-to-individual and individual-to-group analysis method and introduce the three-step intra-subject longitudinal 
approach. We further exemplify the new approach with longitudinal research of three-moment temporal dynamics and shortly discuss the merits and limitations of the intra-subject approach before applying it to a longitudinal team study presented in Chapter 5.

\section{Two Approaches to Analyze Temporal Dynamics}

\section{Inter-subject Longitudinal Approach}

In the inter-subject longitudinal approach, group-level temporal dynamic pattern is first estimated; and then individual patterns are inferred. Developed in crosssectional research, the inter-subject approach is widely applied in longitudinal research. For example, when studying organizational newcomers' feedback seeking behavior, Chan (1998), using simulated data, first estimates the intercept and slope that best fits the sample's change trajectory and then infers the intercepts and slopes of individuals' change trajectories from the sample distribution. Later, he establishes the causal links between the temporal dynamics of feedback seeking behavior and the neuroticism antecedent by examining the association between inter-person differences in the intercept and slope of feedback seeking behavior and inter-person differences in neuroticism levels. Likewise, in a study on the impact of team conflict over time on team performance, Jehn and Mannix (2001) first examine the association between inter-team differences in conflict level at respective time moments and inter-team differences in performance level and then delineate the team conflict dynamic pattern of high-performing teams and low-performing teams by the subsample's mean level of team conflict at respective time moments. In both studies, individual subjects' (i.e. individual persons' or individual teams') temporal dynamic patterns of the studied attribute are considered as either randomly deviant from the group's (i.e. the sample's or sub-sample's) pattern or identical to each other and to the generic group's pattern. The underlying logic of the analyses is that individuals follow identical temporal dynamic pattern until the opposite evidence shows.

However, using the inter-subject approach to study intra-subject attribute change over time and its relations to antecedents and consequences neither examines the intended theoretical inquiry of causality nor corresponds to the empirical reality. Research on the antecedents of intra-subject attribute change over time examines what antecedents lead to different individual developmental patterns of an attribute over time and to what degree antecedent levels determine intra-subject attribute change patterns over time. Likewise, research on the consequences of intra-subject attribute change over time examines what consequences result from different individual developmental patterns of an attribute over time and to what degree intra-subject attribute change patterns over time affect consequence levels. In both inquiries, intra-subject attribute temporal dynamics should be considered as a holistic phenomenon, and conceptualized and studied accordingly, i.e. as tempo- 
rally integrated within a subject and undividable by discrete time moments within a subject. The homogeneity assumption of the inter-subject approach does not acknowledge inter-subject differences in intra-subject attribute dynamic patterns over time; the group-to-individuals decomposition analysis method does not examine intra-subject attribute dynamics over time as a holistic phenomenon and concept. A compatible research approach to the research question should first examine each subject's attribute developmental pattern over time and then compare individual patterns, as a whole, across subjects.

Furthermore, the homogeneity assumption and group-to-individuals method do not always correspond to the empirical reality. For example, in team processes research, several empirical observations have shown that teams differ largely in their process dynamics over time. When studying project teams' functioning in real time, researchers find that project teams' transition from conceptualizing project ideas to implementing the ideas - initially termed midpoint transition-may occur before, at, or after the exact middle time point of team projects (Chang, Bordia, \& Duck, 2003; Gersick, 1989; Okhuysen \& Waller, 2002). Likewise, Raes (2009) finds that teams differ significantly in conflict dynamics and trust development over time. Therefore, using the inter-subject approach to study idiosyncratic team interactions over time may exclude rich information about what actually happens in each team over time and how heterogeneous team process dynamic patterns over time relate to heterogeneous team input levels and/or team output levels. In sum, the incompatibility between the intended theoretical inquiry and the research method, and between the researched phenomenon and the research method may impede researchers from building valid and strong theories. Moreover, the potential harm of such incompatibility may aggravate as the number of measurement moments increases and when research involves the nomological networks (i.e. antecedents and/or consequences) of the temporal dynamics of an attribute (Roe, 2008).

\section{Intra-Subject Longitudinal Approach}

We develop an intra-subject longitudinal approach from a reverse heterogeneity assumption that individuals may differ in the dynamic pattern of a psychological attribute over time. Hence, subgroup-level pattern is the pattern of a set of sufficiently similar individual patterns; group-level "pattern" is the distribution of individual patterns in different subgroups, in other words, the number of individual cases in each subgroup. By constructing subgroup-level and group-level pattern from possibly heterogeneous individual patterns, we preclude collapsing individual patterns into one generic pattern and consequently losing rich information about idiosyncratic individual attribute dynamics over time.

The proponents for using the inter-subject approach in longitudinal research may argue that the variances-based approach is robust and works well in crosssectional research. We, however, argue that a robust yet "wrong" approach has rather limited contribution to building valid and strong theories. The trade-off be- 
tween a model's validity and robustness, although may not be considered so important in cross-sectional research, is utmost critical in longitudinal research. The homogeneity assumption and group-to-individual method of the inter-subject approach may lead researchers to overlook systematic and ecologically-valid differences among individual subjects. Therefore, researchers may need to either empirically verify the homogeneity assumption before applying the inter-subject approach in longitudinal studies or apply an alternative intra-subject approach that regards intra-subject attribute dynamics over time as a holistic phenomenon and concept, acknowledges possibly heterogeneous individual dynamic patterns, and construct group-level pattern from the distribution of heterogeneous individual patterns. Next, we elaborate the three steps of the intra-subject approach via an example study of temporal dynamics defined by three measurement moments.

\section{Intra-Subject Longitudinal Analysis}

The intra-subject longitudinal approach consists of three steps. In this chapter we will exemplify the approach with temporal dynamics defined by three measurement moments. We choose the three-moment temporal dynamics for two reasons. First, it is the simplest longitudinal research design and therefore provides the easiest scenario to elaborate the newly developed approach. Methodological complexity increases as the number of measurement moments increases. We discuss the application of the intra-subject approach in studying multiple-moment temporal dynamics elsewhere. Second, although the intra-subject approach can be generally applied in the research of intra-subject attribute change over time, the commonly used InputProcess-Output (IPO) framework in team research, the nonlinear team development model (e.g. punctuated equilibrium model, Gersick, 1988, 1989, 1991), and the temporal taxonomy of team processes (Marks, Mathieu, \& Zaccaro, 2001) provide a theoretical platform in which the intra-subject approach can be applied and help to advance extant theories. Longitudinal team research often contains repeated measurement with a small number of time moments, three being the modus (Roe, 2009). The punctuated equilibrium model, a primary team development theory developed from real-time observation of project teams, suggests, the beginning, midpoint, and end of team projects are three critical time moments of team functioning. Therefore, it is not only convenient but also theoretically grounded to apply the intra-subject approach to three-moment temporal dynamics.

\section{Define Temporal Dynamics}

Parameters. A basic temporal dynamic pattern, that is, a pattern defined by one subject's attribute score change between two consecutive time moments, is characterized by three parameters, that is, change direction, change degree, and change rate. Change direction refers to the tendency of a subject's attribute score change between two time moments, including increase, decrease, or constant (i.e. being 
stable). Accordingly, in the consequent analysis, the variable of attribute change direction can be coded as positive, negative, or zero, respectively. Change degree refers to the absolute score difference between two time moments. For example, a five-Celsius-degree temperature increase between 9:00 a.m. and 10:00 a.m. and a five-Celsius-degree decrease between 7:00 p.m. and 8:00 p.m. are considered to have the same change degree, that is, a five-Celsius-degree difference an hour. Change rate refers to the relative score difference between time moments. In a sense, it is a combination of change direction and change degree. In the above example, the five-Celsius-degree increase between 9:00 a.m. and 10:00 a.m. has a positive change rate of five degrees an hour, and the five-Celsius-degree decrease between 7:00 p.m. and 8:00 p.m. has a negative change rate of minus five degrees an hour.

A taxonomy of three-moment temporal dynamics. A three-moment temporal dynamic pattern contains three consecutive time moments (i.e. $\mathrm{t}_{0}, \mathrm{t}_{1}, \mathrm{t}_{2}$ ) or two consecutive time intervals (i.e. $t_{0}-t_{1}, t_{1}-t_{2}$ ). It can be further characterized by contrasting each of the three dynamics parameters between $t_{0}-t_{1}$ and $t_{1}-t_{2}$. Such contrasting results in a taxonomy of 17 temporal dynamic patterns listed in Table 4.1.

Pattern 1 is an increase-increase pattern with a smaller change degree in the second interval than in the first one; Pattern 2 is an increase-increase pattern with an equal change degree in the second interval as in the first one (virtually a straight upward line); Pattern 3 is an increase-increase pattern with a larger change degree in the second interval than in the first one; Pattern 4 is an increase-decrease pattern with a smaller change degree in the second interval than in the first one; Pattern 5 is an increase-decrease pattern with an equal change degree in the second interval as in the first one; Pattern 6 is an increase-decrease pattern with a larger change degree in the second interval than in the first one; Pattern 7 is a decrease-increase pattern with a smaller change degree in the second interval than in the first one; Pattern 8 is a decrease-increase pattern with a smaller change degree in the second interval than in the first one; Pattern 10 is a decrease-decrease pattern with a smaller change degree in the second interval than in the first one; Pattern 11 is a decrease-decrease pattern with an equal change degree in the second interval as in the first one (virtually a straight downward line); Pattern 12 is a decrease-decrease pattern with a larger change degree in the second interval than in the first one; Pattern 13 is a stableincrease pattern; Pattern 14 is a stable-decrease pattern; Pattern 15 is a horizontal straight line ${ }^{24}$; Pattern 16 is an increase-stable pattern; Pattern 17 is a decreasestable pattern.

\footnotetext{
${ }^{24}$ We define an attribute "being stable" as having identical attribute level over time. We consider this definition as suitable to team research, because most team process measures are five or seven Likertpoint scales.
} 


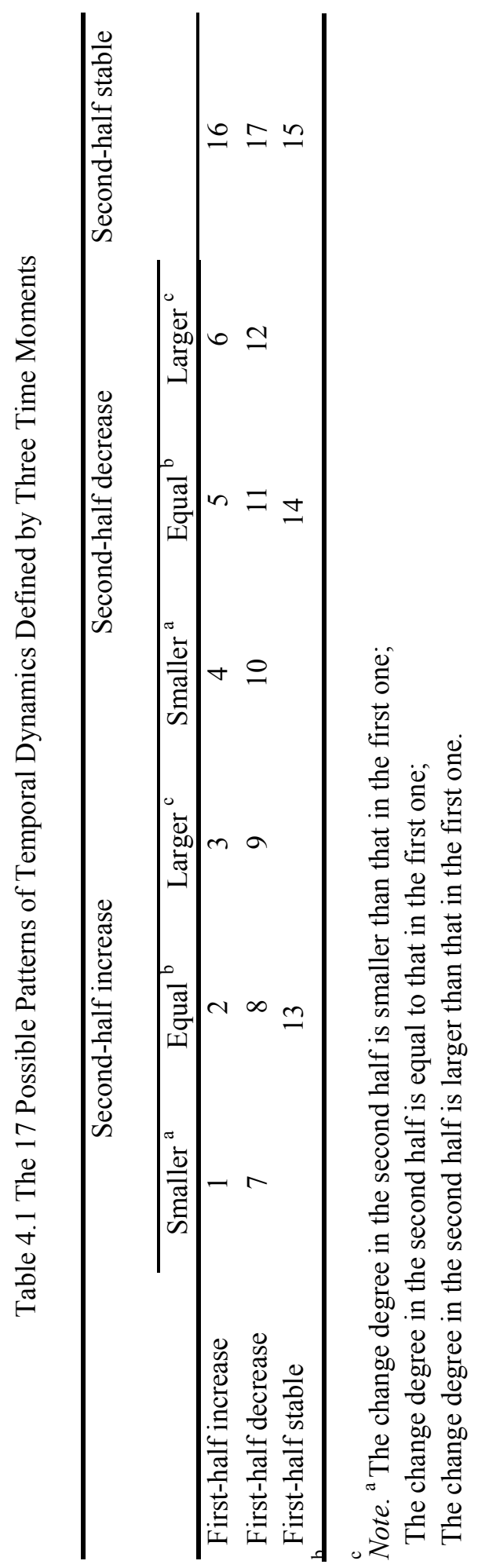


The 17 temporal dynamic patterns can also be described in the mathematical terms. One three-moment temporal dynamic pattern can be written in a quadratic function $\mathrm{f}(\mathrm{t})=\mathrm{at}^{2}+\mathrm{bt}+\mathrm{c}$. The letter $\mathrm{t}$ represents time moment $(\mathrm{t}=0,1,2)$; $\mathrm{f}(\mathrm{t})$ represents attribute score. The sign (i.e. positive, negative, zero) of the first-order derivative function $\mathrm{f}^{\prime}(\mathrm{t})=2 \mathrm{at}+\mathrm{b}$, from one time moment to another indicates change direction (i.e. increase, decrease, constant) between the two time moments. The sign (i.e. positive, negative, zero) of the second-order derivative function $f^{\prime \prime}(t)=2 a$ indicates the tendency of change rate (i.e. upward, downward, constant) from $t_{0}$ to $t_{2}$. Change rate is the relative difference of attribute score from one time moment to another. A positive $f^{\prime}$ ' $(t)$ from $t_{0}$ to $t_{2}$ implies an upward change rate, meaning that change rate is increasing between the two time intervals. Depicted in a Euclidian plane, patterns with an upward change rate are convex patterns, including increase patterns with an increasing change degree, U-shape patterns, and decrease patterns with a decreasing change degree. A negative $f^{\prime}$ ' $(t)$ from $t_{0}$ to $t_{2}$ implies an downward change rate, meaning that the change rate is decreasing between the two time intervals. In a Euclidian plane, patterns with a downward change rate are concave patterns, including increase patterns with a decreasing change degree, inverted-U-shape patterns, and decrease patterns with an increasing change degree. Finally, a zero $f^{\prime \prime}(t)$ from $t_{0}$ to $t_{2}$ implies a constant change rate. Patterns with a constant change rate include increasing lines, decreasing lines, and horizontal lines. We summarize the 17 temporal dynamic patterns described in the mathematical language in Table 4.2. 


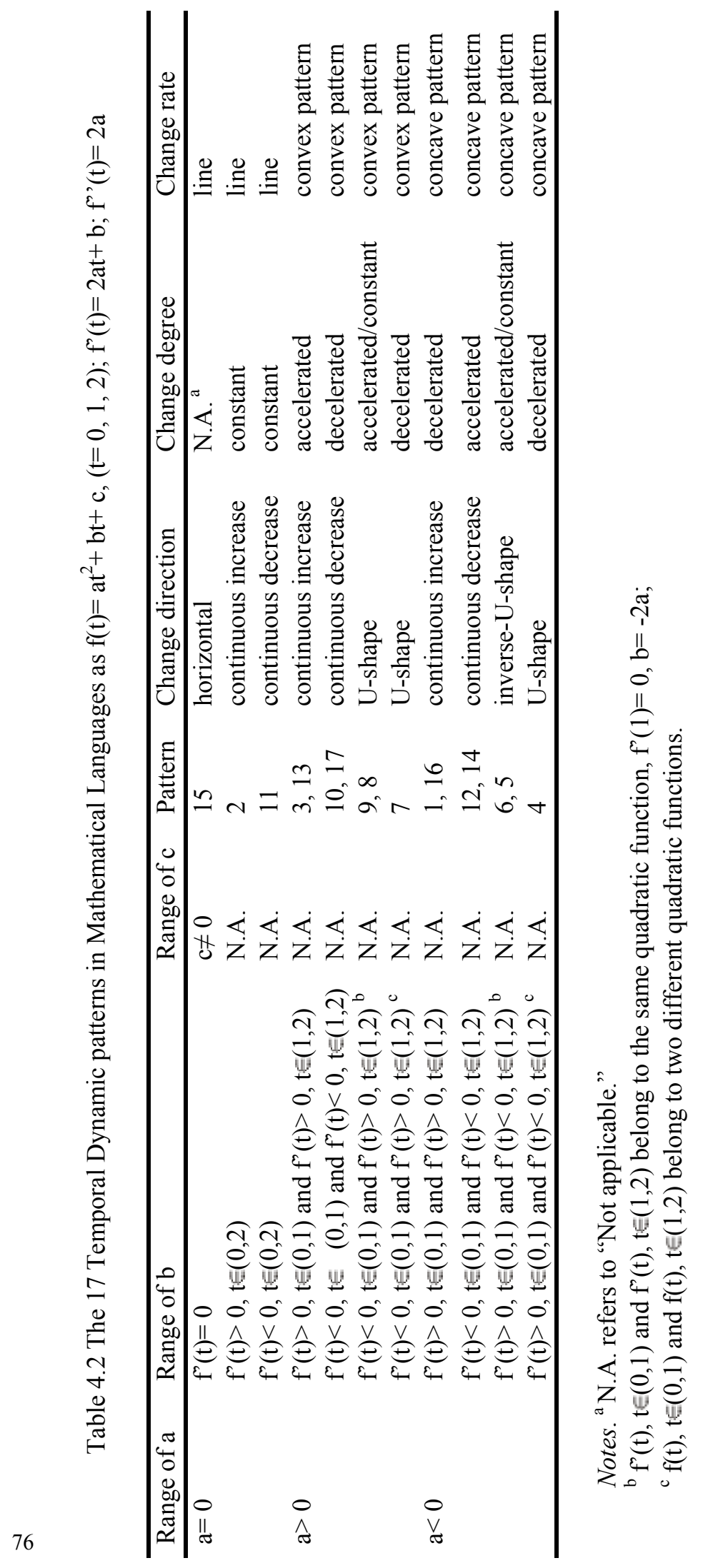


When $f^{\prime \prime}(t)=0, f^{\prime}(t)=0, t \in(0,2)$ and $c \neq 0$, the quadratic function is a horizontal line from $t_{0}$ to $t_{2}\left(\right.$ Pattern 15). When $\mathrm{f}^{\prime \prime}(\mathrm{t})=0$ and $\mathrm{f}^{\prime}(\mathrm{t})>0, \mathrm{t} \in(0,2)$, the quadratic function is an increasing line from $\mathrm{t}_{0}$ to $\mathrm{t}_{2}$ (Pattern 2); when $\mathrm{f}^{\prime}{ }^{\prime}(\mathrm{t})=0$ and $\mathrm{f}^{\prime}(\mathrm{t})<0$, $\mathrm{t} \in$ $(0,2)$, the quadratic function is a decreasing line from $t_{0}$ to $t_{2}$ (Pattern 11).

When $f^{\prime \prime}(t)>0$, the quadratic function denotes an upward change rate and convex patterns from $t_{0}$ to $t_{2}$. Particularly, when $f^{\prime}(t)>0, t \in(0,1)$ and $f^{\prime}(t)>0, t \in(1$, 2 ), the quadratic function denotes an accelerated increase pattern from $t_{0}$ to $t_{2}$ (Pattern 3, Pattern 13; Pattern 13 is considered as a special case of Pattern 3, explanation seen below); when $\mathrm{f}^{\prime}(\mathrm{t})<0, \mathrm{t} \in(0,1)$ and $\mathrm{f}^{\prime}(\mathrm{t})<0, \mathrm{t} \in(1,2)$ the quadratic function denotes a decelerated decrease pattern from $t_{0}$ to $t_{2}$ (Pattern 10, Pattern 17; Pattern 17 is considered as a special case of Pattern 10); when $\mathrm{f}^{\prime}(\mathrm{t})<0, \mathrm{t} \in(0,1)$ and $f^{\prime}(t)>0, t \in(1,2)$, in other words, $f^{\prime}(1)=0$, the quadratic function denotes a U-shape pattern from $t_{0}$ to $t_{2}$ (Pattern 6, Pattern 5; Pattern 5 is considered as a special case of Pattern 6); and when $f^{\prime}(t)<0, t \in(0,1)$ and $f^{\prime}(t)>0, t \in(1,2)$, in other words, $f(t), t \in$ $(0,1)$ and $f(t), t \in(1,2)$ belong to two different quadratic functions, which together denote a U-shape pattern from $t_{0}$ to $t_{2}$ (Pattern 7).

When $\mathrm{f}^{\prime \prime}(\mathrm{t})<0$, the quadratic function denotes a downward change rate and concave patterns from $t_{0}$ to $t_{2}$. Particularly, when $f^{\prime}(t)>0, t \in(0,1)$ and $f^{\prime}(t)>0, t \in$ $(1,2)$, the quadratic function denotes a decelerated increase pattern from $t_{0}$ to $t_{2}$ (Pattern 1, Pattern 16; Pattern 16 is considered as a special case of Pattern 1); when $\mathrm{f}^{\prime}(\mathrm{t})<0, \mathrm{t} \in(0,1)$ and $\mathrm{f}^{\prime}(\mathrm{t})<0, \mathrm{t} \in(1,2)$, the quadratic function denotes an accelerated decrease pattern from $t_{0}$ to $t_{2}$ (Pattern 12, Pattern 14; Pattern 14 is considered as a special case of Pattern 12); when $f^{\prime}(t)>0, t \in(0,1)$ and $f^{\prime}(t)<0, t \in(1,2)$, in other words, $\mathrm{f}^{\prime}(1)=0$, the quadratic function denotes an inverted-U-shape pattern from $\mathrm{t}_{0}$ to $t_{2}$ (Pattern 9, Pattern 8; Pattern 8 is considered as a special case of Pattern 9); when $f^{\prime}(t)>0, t \in(0,1)$ and $f^{\prime}(t)<0, t \in(1,2)$, in other words, $f(t), t \in(0,1)$ and $f(t)$, $t \in(1,2)$ belong to two different quadratic functions, which together denote an inverted U-shape pattern from $t_{0}$ to $t_{2}$ (Pattern 4 ).

Pattern 13 is considered as a special case of Pattern 3, because its overall pattern monotonically increases from $t_{0}$ to $t_{2}$ and its change degree in the second half is larger than in the first; Pattern 17 is considered as a special case of Pattern 10, because its overall pattern monotonically decreases from $t_{0}$ to $t_{2}$ and its change degree in the second half is smaller than in the first; Pattern 16 is considered as a special case of Pattern 1, because its overall pattern monotonically increases from $t_{0}$ to $t_{2}$ and its change degree in the second half is smaller than in the first; Pattern 14 is considered as a special case of Pattern 12, because its overall pattern monotonically decreases from $t_{0}$ to $t_{2}$ and its change degree in the second half is larger than in the first. Pattern 5 is considered as a special case of Pattern 6 rather than Pattern 4, because we prefer to define it by one quadratic function rather than two; for the same reason, Pattern 8 is considered as a special case of Pattern 9 rather than of Pattern7. 
Therefore, each pattern can be described in the mathematical language. For example, Pattern 1 and 16 are decelerated monotonic increasing concave patterns. Pattern 2 is an increasing line. Pattern 3 and 13 are accelerated monotonic increasing convex patterns. Pattern 4 is a decelerated inverted-U-shape convex pattern. Pattern 5 is an inverted-U-shape concave pattern with a constant change degree. Pattern 6 is an accelerated inverted-U-shape concave pattern. Pattern 7 is a decelerated U-shape concave pattern. Pattern 8 is a U-shape convex pattern with a constant change degree. Pattern 9 is an accelerated U-shape convex pattern. Pattern 10 and 17 are decelerated monotonic decreasing convex patterns. Pattern 11 is a decreasing line. Pattern 12 and 14 are accelerated monotonic decreasing concave patterns.

Simplify the taxonomy. The 17-pattern temporal dynamics taxonomy presents the most fine-grained categorization of three-moment temporal dynamic patterns, which can be further simplified by aggregating the 17 patterns based on each of the three dynamics parameters. Based on change direction, the 17 patterns are grouped into five broader categories, that is, monotonically increasing patterns (Pattern 1, 2, $3,13,16$ ), monotonically decreasing patterns (Pattern 10, 11, 12, 14, 17), U-shape patterns (Pattern 4, 5, 6), inverted-U-shape patterns (Pattern 7, 9, 8), and a horizontal pattern (Pattern 15). We present this change-direction-based categorization scheme in Figure 4.1.

Figure 4.1 The 17 Possible Patterns of Temporal Dynamics Defined by Three Time Moments (Categorized by Change Direction)

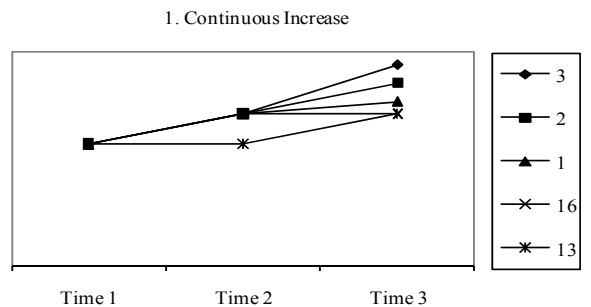


2. Inverted-UShape

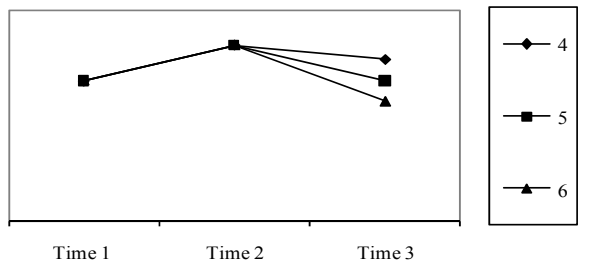

3. U Shape

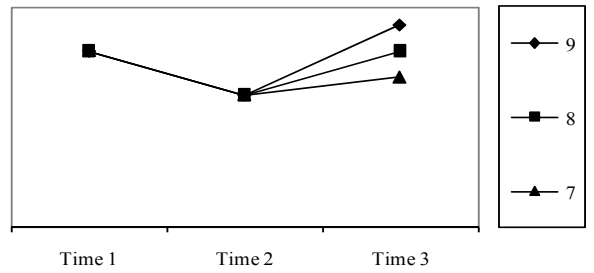

4. Continous Decrease

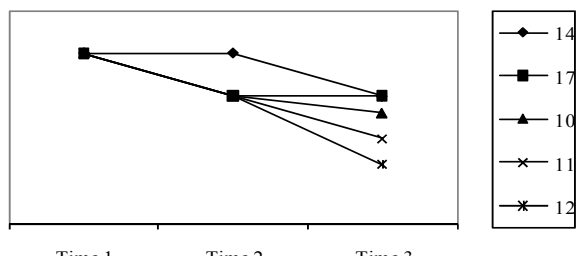

$\begin{array}{lll}\text { Time } 1 & \text { Time } 2 & \text { Time } 3\end{array}$

5. Stable

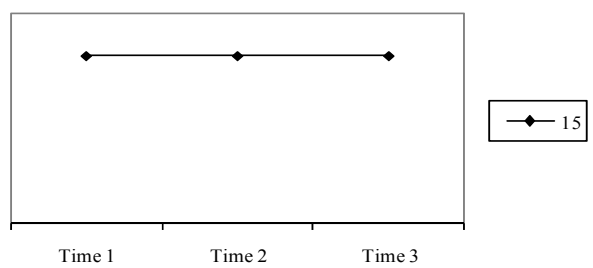


Based on change degree, more specifically, on contrasting change degree between two time intervals, the 17 patterns are grouped into three broader categories, that is, patterns with an accelerating change degree (Pattern 3, 6, 9, 12, 13, 14), patterns with a decelerating change degree (Pattern 1, 4, 7, 10, 16, 17), and patterns with a constant change degree (Pattern 2, 5, 8, 11, 15). The accelerating change degree indicates the change degree in the second time interval is larger than that in the first one; the decelerating change degree indicates the change degree in the second time interval is smaller than that in the first one. We present this changedegree-based categorization scheme in Figure 4.2.

Figure 4.2 The 17 Possible Patterns of Temporal Dynamics Defined by Three Time Moments (Categorized by Change Degree)
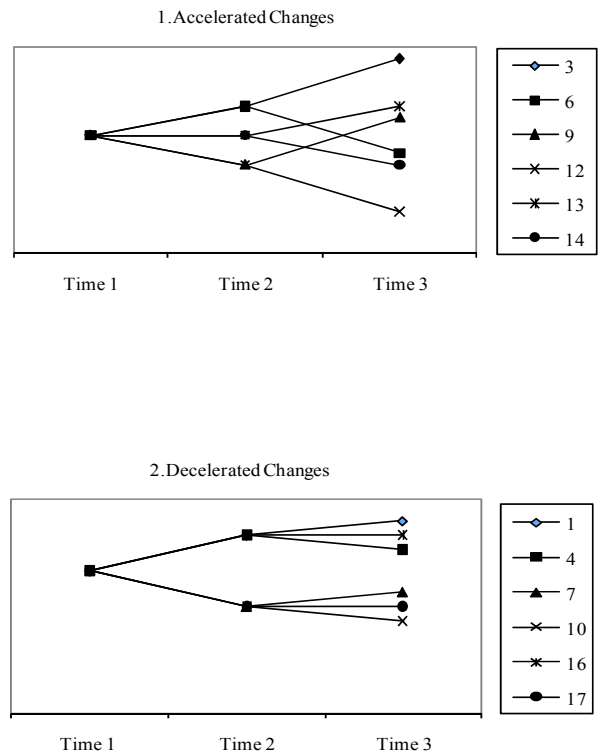


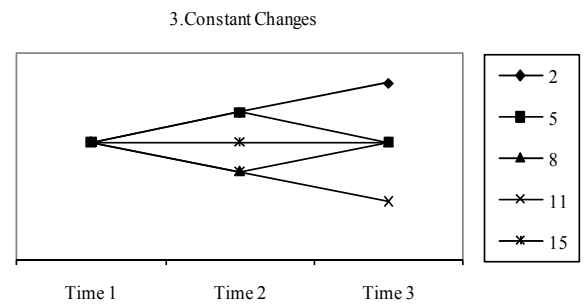

The third categorization scheme is based on change rate, according to which the 17 patterns are grouped into three categories, that is, convex curves, concave curves, and straight lines. Convex patterns are the patterns in which change rate in the second time interval surpasses that in the first one. These patterns include accelerated increase patterns, U-shape patterns, and decelerated decrease patterns (Pattern $3,4,8,9,10,13,17)$. Concave patterns are the patterns in which change rate in the second time interval lags behind that in the first one. These patterns contain decelerated increase patterns, inverted-U-shape patterns, and accelerated increase patterns (Pattern 1, 5, 6, 7, 12, 14, 16). Straight lines are the patterns that demonstrate constant change rates (Pattern 2, 11, 15). We present this change-rate-based categorization scheme in Figure 4.3.

Figure 4.3 The 17 Possible Patterns of Temporal Dynamics Defined by Three Time Moments (Categorized by Change Rate)

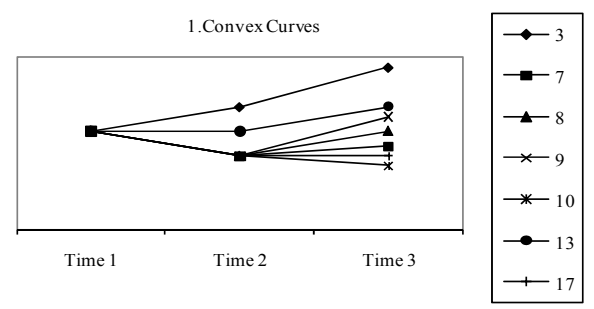



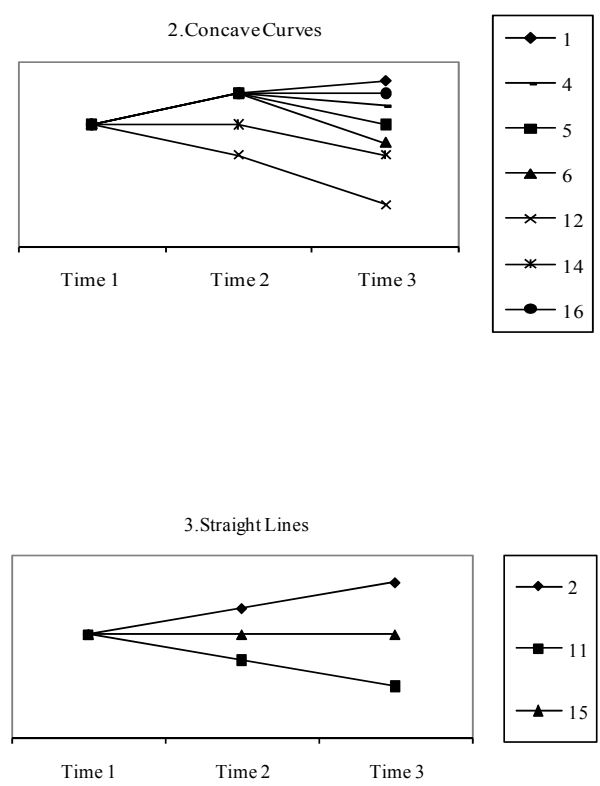

\section{Step 1: Depict Temporal Dynamics}

The first step of the intra-subject approach is to calculate, for each subject, $Z$-scores of the attribute scores across three time moments. The intra-subject intertemporal Zscores therefore denote subjects' temporal dynamic pattern of the attribute, regardless of subjects' actual attribute scores at respective time moments.

\section{Step 2: Cluster Individual Subjects by Temporal Dynamics}

Use the $Z$-scores to perform hierarchical clustering analysis with cosine distance and within-cluster similarity algorithm in the Statistical Package for Social Sciences (SPSS). Cosine distance allows us to cluster subjects by the shape of subjects' Zscores, rather than by the actual level of the Z-scores as when the Euclidean distance is chosen. The within-cluster similarity algorithm ensures that subjects with the most similar $Z$-score shape (i.e. the temporal dynamic pattern of the attribute) are clustered together first. As a result, this hierarchical clustering analysis generates clusters of individual subjects based on their attribute dynamic patterns. These clusters can be further represented in one categorical variable for the analysis in the third step. 


\section{Step 3: Link Temporal Dynamics to Antecedents and Consequences}

After obtaining the dynamics-based categorical variable, we are able to examine the nomological networks of intra-subject attribute dynamics over time with its antecedents and consequences by incorporating the categorical variable into traditional multivariate analysis techniques. For example, we can use multinomial probit regressions or linear regression with dummy variables to examine what antecedents lead to particular types of temporal dynamics of a studied attribute. Likewise, we can use Multivariate Analysis of Variances (MANOVA) to examine what consequences result from particular temporal dynamic patterns of the attribute. By entering the dynamics-based categorical variable into traditional multivariate analysis techniques, we are able examine intra-subject attribute dynamics over time as a gestalt phenomenon, concept, and variable. In Chapter 5, we present a longitudinal team study, in which the intra-subject approach is applied in analogue to the intersubject approach.

\section{Conclusion}

In this chapter, we developed an intra-subject longitudinal approach that regards intra-subject attribute dynamics over time as a holistic and temporally-integrated phenomenon and concept, acknowledges possibly heterogeneous individual dynamic patterns over time, and infers group-level dynamic patterns from the distribution of emergent individual patterns. The newly developed approach bears both merits and limitations. On the one hand, the approach helps to answer the research questions, in which subjects' attribute dynamics over time is conceptualized as a whole. It also represents the empirical reality better than the inter-subject approach does. On the other hand, processes-based models estimated by the intra-subject approach may be less robust than the variances-based models estimated by the inter-subject approach, since the dynamics-based categorical variable requires more degrees of freedom. We, however, question the added value of robust yet less valid research findings for advancing extant knowledge and theories, given the incompatibility between the purpose of the theoretical inquiry and the research approach, and between the researched phenomena and the research approach. In Chapter 5, we examine the impact of team conflict over time on team performance and team satisfaction with both the intra-subject approach and the inter-subject approach and report differential findings. 



\section{Chapter 5}

\section{The Impact of Team Conflict over Time on Team Performance and Team Satisfaction: The Intra-Subject versus Inter-subject Longi- tudinal Approach ${ }^{25}$}

Team conflict has been considered as one of the most influential team processes on team performance and team satisfaction (Kozlowski \& Ilgen, 2006). Team conflict consists of three forms, that is, task conflict, relationship conflict, and process conflict (Jehn \& Mannix, 2001). Although empirical studies have shown both beneficial and detrimental effect of team conflict on team performance and team satisfaction (Eisenhardt \& Bourgeois, 1988; Janis, 1972; Pelled, 1996), recent metaanalyses have concluded the overall detrimental effect of team conflict on the two team outcomes (De Dreu \& Weingart, 2003; De Wit \& Greer, 2008). Furthermore, recent research based on Gersick;s $(1988,1989,1991)$ punctuated equilibrium team development model has shown the "typical" team conflict development patterns that are associated with high- and low-performing teams and the two subsample patterns are significantly differ from each other (Jehn \& Mannix, 2001). However, given the logical issues of the inter-subject approach discussed in Chapter 4, we are cautious to conclude that all the high-performing or low-performing teams would follow the "typical" conflict development patterns over time.

In a longitudinal team study, we examine the impact of team conflict over time on team performance and team satisfaction using both the intra-subject longitudinal approach and the inter-subject longitudinal approach. In a sample of 42 project teams of business graduate students, we find that team conflict over time relates differently to team satisfaction when the effect is examined by the two approaches. For example, results from the intra-subject approach suggest that the developmental pattern of team relationship conflict throughout team projects affects

\footnotetext{
${ }^{25}$ An early version of this chapter was presented at the 2009 Annual Meeting of the Academy of Management in Chicago, Illinois, the United States. An updated version is currently under review at a peerreviewed journal (c.f. Li, J., \& Roe, R.A., Studying team conflict over time: An intra-subject versus inter-subject longitudinal approach, Manuscript submitted for publication.)
} 
team satisfaction at the end of team projects, whereas results from the inter-subject approach suggest that only team relationship conflict in the first half of the project time affects team final satisfaction. The differential findings suggest that the two approaches address distinct aspects pertaining to the impact of team conflict over time on team satisfaction, which are to be explained by distinct theories. 
Team processes are team internal and external interactions over time that explain how team inputs affect team outputs. A recent development in team research is to conceptualize team processes as dynamic phenomena and accordingly measure them repeatedly over time (Gersick, 1988, 1989; Ilgen, Hollenbeck, Johnson, \& Jundt, 2005; Marks, Mathieu, \& Zaccaro, 2001). Punctuated equilibrium model, a primary team development model developed from real-time observation (Gersick, 1988, 1989, 1991), suggests that project teams working under predefined deadlines accomplish tasks in a non-linear fashion over time (Chang, Bordia, \& Duck, 2003; Gersick, 1988; Seers \& Woodruff, 1997), and that teams that successfully transition from an inertial state of conceptualizing project ideas to an active state of implementing the project ideas at the exact middle time point outperform those who do not (Gersick, 1989).

Drawing upon the punctuated equilibrium model, we argue that task accomplishment as the primary goal and activity of project teams will elicit and, to substantial extent, affect other specific team processes (e.g. conflict, communication). The elicited specific team processes can be seen as secondary goals and activities in the teams, that facilitate the accomplishment of team tasks. Consequently, the progress of team tasks over time will entrain the developmental patterns of the elicited team processes over time. It implies that (1) the elicited team processes develop nonlinearly over time and (2) teams' idiosyncratic dynamic patterns of the elicited team processes may lead to different team output levels. A few previous studies have shown the non-linear developmental patterns of elicited team processes over time and their associations with different team output levels. For example, Ericksen and Dyer (2004) find that project teams' mobilization strategies at the early stage of team projects affect their final performance levels. Jehn and Mannix (2001) find that high-performing teams are characterized by an inverted-U-shape developmental pattern of task conflict, a stable developmental pattern of relationship conflict, and a U-shape developmental pattern of process conflict over time. However, as discussed in Chapter 1 (p.12-14), there are several methodological limitations in current empirical team processes research. Studies on the linearity or nonlinearity of team process dynamics are rather descriptive than examining causality (Hackman, 1987). Studies on the impact of team process dynamics often rely on qualitative case studies or the inter-subject longitudinal approach to establish causality. The qualitative case study method, although valuable for theory development, is less rigorous and therefore less suitable for theory testing than statistical inferences. The inter-subject approach aligns with neither the research questions that regard team process dynamics over time as a holistic phenomenon and concept, nor the empirical reality that teams may differ in their internal and external interactions over time.

The aim of this chapter is to examine the impact of team conflict dynamics over time on team performance and team satisfaction via the intra-subject and intersubject longitudinal approach and compare the results. We report differential results from the two approaches, which resonates organization theorists' (McGrath \& 
Tschan, 2004; Roe, 2009; Van de Ven, 2007) recent but urgent call for applying both processes-based models and variances-based models in building strong organization theories. We extend the punctuated equilibrium model (Gersick, 1988; 1989) by examining the causality of team conflict dynamics over time, as a holistic phenomenon and concept, and team output level via statistical inferences.

\section{Theoretical Background}

\section{Punctuated Equilibrium Model and the Temporal Dynamics of Team Processes}

Non-linear team process dynamics over time. As the punctuated equilibrium model (Chang et al., 2003; Seers \& Woodruff, 1997; Gersick, 1988, 1989, 1991) and temporal taxonomy of team processes (Marks et al, 2001) suggests, project teams working under predefined deadlines accomplish tasks in a nonlinear fashion over time. The task accomplishment process contains two transition stages ${ }^{26}$ and two action stages. The first transition stage is the first one or two team meetings, in which team members brainstorm possible ideas for the final project solutions, set goals to achieve, and develop norms to progress the team tasks over time. After the initial meetings, teams enter the first action stage in which they continue to develop the project ideas and remain in a rather inertial state until entering the second transition stage. In the second transition stage, teams realize the unsatisfactory task progress in the past and reorient themselves towards accomplishing the remaining tasks. The second action stage starts from the team reorientation and lasts until the end of projects. In this stage, teams implement the project ideas developed in the previous stages and work intensively to transform the conceptual ideas into deliverable solutions.

Drawing upon the model and taxonomy, we argue that the development of specific team processes over time is largely determined by the progress of the team tasks over time, following a non-linear fashion. As Gersick (1991) and Marks and colleagues (2001) note, project teams alternate between action stages fraught with gradual changes and transition stages fraught with drastic changes. The level of a transition-related team process (e.g. planning) may be high in a transition stage but can diminish in an action stage; vice versa, the level of an action-related process (e.g. coordination) may be high in an action stage but can diminish in a transition stage. As team tasks advance nonlinearly over time through transition and action stages, specific team processes elicited by team task accomplishment may, accordingly, develop nonlinearly over time.

\footnotetext{
${ }^{26}$ In this dissertation, we distinguish between "stage" and "phase" in team development over time. "Stage" implies that team development is defined by critical team events, such as team transition; "phase" implies that team development is defined by the elapse of real time, such as the middle time point of a project.
} 
Idiosyncratic team process dynamics and its impact on team outputs. The second aspect of the punctuated equilibrium model suggests that whether project teams successfully reorient themselves at the exact middle time point of team projects or not influences their final performance. Project teams differ in the timing of the second transition (i.e. the in-project transition) and consequently differ in the pattern of task progress over time. The inter-team differences in task progress over time, in turn, lead to inter-team differences in team performance levels at the end. Logically, three possible team transitions may occur, that is, team transition can happen before, at, or after the exact middle time point. The punctuated equilibrium model does not suggest that project teams pace their work at a constant speed over time. "Seemingly" constantly pacing teams may have the same working intensity at both the idea conceptualization and idea implementation stage, but smoothly change the nature of the team work during the midpoint transition. Chang and colleagues (2003) have observed that nine out of the total 25 studied project teams underwent team transition at the exact midpoint; 12 before and after it; four teams reported no transition at all.

At-midpoint transition teams tend to outperform the other teams due to a wellbalanced time usage between conceptualizing project ideas and implementing them (Gersick, 1989). We therefore argue that project teams, whose temporal dynamics patterns of elicited team processes reflect the occurrence of midpoint transition, tend to outperform the other teams. To our knowledge, most empirical studies on real-time team development address mostly the time-linearity or non-time-linearity question (e.g. Chang and colleagues' [2003], Gersick's [1988, 1989], and Seers and Woodruff's [1997] studies). We go one step further in this study and examine the impact of team conflict dynamics over time, as a holistic phenomenon and concept, on team performance and team satisfaction. Team conflict is chosen, because it is one of the most studied team processes and empirical evidence suggests its impact on team performance and team satisfaction (for an overview, see two meta-analysis studies by De Dreu and Weingart, [2003] and De Wit and Greer [2008]).

\section{Task Conflict over Time}

Task conflict is "an awareness of differences in viewpoints and opinions pertaining to a group task (Jehn \& Mannix, 2001: 238)." Brainstorming possible ideas for the final project solutions in the initial team meetings can trigger task conflict. The more diverse team members are in informational demographics (e.g. functional background), the more task conflict may result (De Wit \& Greer, 2008).

At the exact middle time point, project teams are either undergoing the second transition, have already completed the transition, or have not experienced the transition yet. For the midpoint in-transition teams, task conflict may be higher than that at the beginning. As Gersick (1989) suggests, project teams' midpoint transition takes place in two ways. In the first way, after assessing the previous work, project teams, unsatisfied with the developed project concepts, start to conceptualize new 
project ideas and brainstorm the implementation of the new project ideas at the same time. Task conflict level may increase from the beginning on, because both the overall project ideas and their implementation are being discussed and considered at the moment. In the second way, after assessing the previous work, project teams, content with their earlier work, simply shift their focus from conceptualizing project ideas to implementing the ideas. Contemplating on idea implementation may trigger more task conflict at this moment than conceptualizing overall project ideas did at the beginning. It is because idea implementation often involves the consideration and fixation of concrete technical details, whereas idea conceptualization only aims at developing general project concepts. For midpoint post-transition teams, task conflict may also increase from the beginning, because implementing project ideas at the moment involves the discussion and realization of technical details and renders more task-related disagreement than conceptualizing overall project ideas at the beginning. For midpoint ante-transition teams, task conflict may decrease from the beginning, because the teams still remain in a rather inertial state and therefore experience less task-related disagreement than brainstorming possible project ideas at the beginning.

At the end, project teams' subtasks have become the most differentiated. Teams have to finalize every detail of every technical aspect of the final project solution and present the solutions to project stakeholders (e.g. customers, supervisors, and investors). Detail finalization and outcome presentation may trigger novel task-related disagreement. For the at-midpoint transition teams, task conflict increases from the midpoint to the end and throughout team projects it increases at a decelerated rate. If teams' midpoint transition takes place through the reconceptualization of project ideas, task conflict increase in the first half of team projects is due to the additional discussion on idea implementation at the midpoint. Task conflict increase in the second half of team projects, on the other hand, is due to the increased number of implementation subtasks and therefore is expected to be less drastic than the first half increase. If teams' midpoint transition takes place by simply shifting from idea conceptualization to idea implementation, task conflict increase in the first half time is due to a qualitative change from conceptualizing project ideas to fixating more and concrete technical details. Task conflict increase in the second half time, on the other hand, is due to the increased number of implementation subtasks and, again, is expected to be less drastic than the first half increase. For the ante-midpoint transition teams, task conflict may decrease from the midpoint to the end, because the early transition allows the teams to have some slack time in the second half of team projects to work on the social aspects of the teams and may help to reduce task-related disagreement in the teams. Therefore, throughout team projects, task conflict may demonstrate an inverted U-shape pattern. For the post-midpoint transition teams, task conflict may demonstrate a Ushape developmental pattern throughout team projects, because of their inertial state and consequently little task-related disagreement at the midpoint. When weighing in 
the four situations, we expect task conflict to increase at a decelerated rate from the midpoint to the end. We then hypothesize that

Hypothesis la Overall, task conflict increases at a decelerated rate throughout team projects.

Task conflict is detrimental to team performance and team satisfaction (De Dreu \& Weingart, 2003; De Wit \& Greer, 2008). The higher the level of task conflict a project team has, the more likely it delivers low performance and reports low satisfaction. To our knowledge, few studied have addressed the impact of team task conflict development over time on team performance and team satisfaction at the end. One exception is Jehn and Mannix's (2001) study that reports an inverted Ushape pattern for high-performing teams and a U-shape pattern for low performing teams. Drawing upon the punctuated equilibrium model, we expect that the temporal dynamics pattern of team task conflict that accompanies midpoint transition is associated with high team performance whereas the other patterns are associated with low team performance. Midpoint transition indicates that project teams may have spent an optimal amount of time between conceptualizing and implementing project ideas and hence result in high team performance. We hypothesize that

Hypothesis $1 b$ Project teams in which task conflict develops over time following a decelerated increase pattern outperform the other teams.

Team satisfaction refers to team members' general attitudes towards working as a team. It is formed by team members' experiences of episodic team activities over time. Therefore, the developmental pattern of team conflict in the second half of team projects may be more influential to team satisfaction at the end than that in the first half of team projects. The ante-midpoint transition teams, thanks to their early transition, may have more time to socialize and deliberately employ conflict management strategies to ameliorate team atmosphere in the second half of team projects. These efforts may consequently reduce task conflict and enhance team members' satisfaction of working as a team (Behfar, Peterson, Mannix, \& Trochim, 2008). In contrast, the at-midpoint transition and post-midpoint transition teams may have to dedicate all the available time in the second half of team projects to implementing the project ideas and have left little time left to work on the social aspects of the teams. Therefore, their task conflict may increase in the second half of team projects and result in team members' low satisfaction level at the end of team projects. We then hypothesize

Hypothesis 1c Project teams in which task conflict develops over time following an inverted U-shape pattern are more satisfied at the end of team projects than the other teams. 


\section{Relationship Conflict over Time}

Relationship conflict is "an awareness of interpersonal incompatibilities, including affective components, such as feeling tension and friction (Jehn \& Mannix, 2001: 238)." Team diversity in social categorical demographics (e.g. gender, age, ethnicity) may lead to relationship conflict at the beginning of team projects. Social identity theory (Tajfel, 1982) suggests that team members tend to identify themselves with others from the same social categories and distinguish themselves from those of other social categories. The in-group identification and out-group distinction explain the initial relationship conflict in the teams.

We expect that, overall, relationship conflict increases throughout team projects. Some researchers such as Pelled, Eisenhardt, and Xin (1999) argue that, over time, the increased familiarity among team members may attenuate the initially perceived differences of team members from different social categories and hence reduce relationship conflict over time. However, their empirical findings do not support the argument and suggest no direct effect of time on team relationship conflict. Drawing upon the punctuated equilibrium model, we reason the opposite, namely, that teams' initial relationship conflict will escalate over time. As time goes by, the increasing workload and mounting pressure in teams to accomplish the projects in time and with accepted quality, increases the interaction frequency of team members from different social categories and leaves teams with less and less time to reconcile the existing relationship conflict. As a result, relationship conflict may aggravate over time. For at-midpoint transition teams, relationship conflict may increase at an accelerated rate throughout the team project, because the teams may have dedicated all the available temporal resources to conceptualizing and implementing project ideas and have no time to reconcile the existing interpersonal tension in the teams. For ante-midpoint transition teams, relationship conflict may demonstrate an inverted-U-shape pattern, increasing from the beginning to the midpoint but decreasing from the midpoint to the end. The early transition permits the teams with some slack time in the second half of team projects to work on the social aspects of the teams, which may relieve the existing interpersonal tension. For postmidpoint transition teams, relationship conflict may demonstrate a U-shape pattern, decreasing from the beginning to the midpoint but increasing sharply from the midpoint to the end. The late transition may indicate that the teams may have spent some time in socializing in the first half of team projects, which may relieve the initial interpersonal tension but delay the crucial in-project transition. The late transition, on the other hand, accumulates enormous pressure in the teams in the second half of team projects to accomplish the projects in time and with accepted quality, which may substantially aggravate the interpersonal tension in the teams. In line with earlier empirical studies (Greer, Jehn, \& Mannix, 2008; Jehn \& Mannix, 2001), we hypothesize that 
Hypothesis $2 a$ Overall, relationship conflict increases at an accelerated rate throughout team projects.

Relationship conflict is detrimental to team performance and team satisfaction (De Dreu \& Weingart, 2003; De Wit \& Greer, 2008). The higher the level of relationship conflict in a project team is, the more likely it delivers low performance and reports low satisfaction. Jehn and Mannix's (2001) find that both highperforming and low-performing teams experience an accelerated increase of relationship over time but to different degree. Based on the punctuated equilibrium model, we argue that the temporal dynamics pattern of relationship conflict linked to the occurrence of midpoint transition is associated with high team performance, whereas the other patterns indicate that project teams do not use all the available temporal resources in accomplishing the team tasks and therefore perform less well.

Hypothesis $2 b$ Project teams in which relationship conflict develops over time following an accelerated increase pattern outperform the other teams.

Whereas team task and process conflict pertain to the content of the team task and the way in which it is carried out, team relationship conflict has a personal anchor and is more sensitive to project teams' deliberate efforts to reconcile interpersonal tension. Project teams' conflict management efforts will lower team relationship conflict and in turn enhance team satisfaction (Behfar et al, 2008). Whereas atmidpoint transition teams may have dedicated all the available temporal resources to accomplishing the team tasks, post-midpoint transition and ante-midpoint transition teams may have spent some time to work on the social aspects of the teams in either the first or second half of team projects, which may reduce relationship conflict and enhance team satisfaction at the end. We hypothesize that

Hypothesis $2 c$ Project teams in which relationship conflict develops over time following a U-shape or inverted U-shape pattern are more satisfied at the end of team projects than teams with the other relationship conflict patterns.

\section{Process Conflict over Time}

Process conflict is "an awareness of controversies about aspects of how task accomplishment will proceed" and "pertains to issues of duty and resource delegation, such as who should do what and how much responsibility different people should get (Jehn \& Mannix, 2001: 239)." Based on the punctuated equilibrium model, we expect that team process conflict develops and affects team performance and team satisfaction in a similar fashion as team task conflict does. In the initial team meetings, project teams discuss the task distribution among individual team members and establish the norms for advancing the team tasks. Such discussion may trigger process conflict. 
For the at-midpoint transition teams, process conflict may increase from the beginning on. If midpoint transition takes place via the reconceptualization of project ideas, process conflict increase is due to the additional discussion on the task distribution and norm establishment for the reconceptualization and upcoming implementation of the reconceptualized project ideas. If midpoint transition takes place via a smooth shift from idea conceptualization to idea implementation, the discussion on the task distribution and norm establishment for the upcoming implementation subtasks may trigger more process conflict than at the beginning, because implementing project ideas involves more and concrete technical details than conceptualizing overall project ideas. For the ante-midpoint transition teams, process conflict may increase, similar to the second way of midpoint transition, due to the discussion on more and concrete technical details. For the post-midpoint transition teams, process conflict may decrease, because the teams still remain in the inertial state of further developing project ideas and have less disagreement on task distribution or norm establishment than when intensively discussing individual responsibility and task advancement at the beginning.

At the end, project teams' subtasks have become most differentiated. Most project teams even form subgroups to work on different aspects of their subtasks (Chang et al., 2003). Delegating numerous subtasks in teams may trigger novel process conflict. For the at-midpoint transition teams, process conflict may increase from the midpoint to the end, but throughout the whole project it may increase at a decelerated rate. If midpoint transition takes place in the first way, process conflict increase in the first half time is due to the additional discussion on the task distribution and norm establishment for the implementation subtasks at the midpoint. Process conflict increase in the second half time, on the other hand, is due to the increased number of implementation subtasks and therefore expected to be less drastic than the first half increase. If midpoint transition takes place in the second way, process conflict increase in the first half time is due to a qualitative change from disagreement related to idea conceptualization procedure to that related to idea implementation procedure. Process conflict increase in the second half time, on the other hand, is due to a mere quantitative increase associated with the increased number of implementation subtasks and therefore expected to be less drastic than the first half increase. For the ante-midpoint transition teams, process conflict may decrease from the midpoint to the end, because the early transition provides the teams with some slack time to work on the social aspects of the teams; this may reduce the process-related disagreement in the second half of project. Therefore, throughout the team project, process conflict is likely to demonstrate an inverted Ushape pattern. For the post-midpoint transition teams, process conflict may increase from the midpoint to the end. Throughout the team project it may demonstrates an U-shape development pattern, because of the teams' inertial state at the midpoint and the low level of process-related disagreement. When weighing in the four situa- 
tions, we expect process conflict to increase at a decelerated rate from the midpoint to the end.

Hypothesis 3 a Overall, process conflict shows a decelerated increase throughout team projects.

Process conflict is detrimental to team performance and team satisfaction (De Wit \& Greer, 2008). The higher the level of process conflict in a project team is, the more likely it delivers low performance and reports low satisfaction. Few studies have addressed the impact of team process conflict development over time on team performance and team satisfaction at the end. Jehn and Mannix's (2001) found a Ushape pattern for high-performing teams and a first-increase-then-stable pattern for low performing teams. Drawing upon the punctuated equilibrium model, we expect that, like task conflict, the temporal dynamics pattern of process conflict that reflects the occurrence of midpoint transition is associated with high team performance, whereas the other patterns are associated with low team performance. Midpoint transition indicates that project teams have spent all the available temporal resources between conceptualizing and implementing project ideas and hence perform better than the other teams. As discussed above, the at-midpoint -transition teams will demonstrate a decelerated increase pattern of process conflict over time. We then hypothesize

Hypothesis $3 b$ Project teams in which process conflict develops over time following a decelerated increase pattern outperform the other teams.

As team satisfaction derives from team members' experiences of episodic team activities throughout team projects, team conflict at a later stage of team projects is likely to have more influence on team satisfaction at the end than team conflict at an earlier stage. The ante-midpoint transition teams, thanks to their early transition, may have more time in the second half of the team projects to work on the social aspects of the teams and to reconcile existing process conflict in the teams. Consequently, these deliberate efforts to manage conflict may enhance team satisfaction at the end of the projects (Behfar, et al, 2008). As discussed earlier, the ante-midpoint transition teams may demonstrate an inverted U-shape pattern of process conflict over time. We hypothesize

Hypothesis 3c Project teams in which process conflict develops over time following an inverted U-shape pattern are more satisfied at the end of team projects than the other teams. 


\section{Methods}

\section{Research Settings, Procedures, and Sample}

To test the hypotheses, we collected data from a business research method course for graduate students in the Faculty of Economics and Business Administration ${ }^{27}$ of Maastricht University during the autumn academic quarter in 2008. A main course assignment for the students was to write research proposals in small teams of three persons and accounted for $30 \%$ of their course grades. Teams were free to choose any business-related topics for the proposals; proposals were graded for the soundness of theoretical reasoning, the feasibility of research methods and the relevancy to business practice. The 7-week course contained an opening lecture in Week 1 and thirteen tutorial meetings spaced evenly from Week 1 to Week 7. Each tutorial group contained maximum 15 students. In Meeting 5, teams presented initial proposals and gathered feedbacks from their tutors and fellow students; In Meeting 13, teams handed in their proposals and presented them in the tutorial groups.

Between the opening lecture and Meeting 1, we collected demographic data via an online questionnaire and randomly assigned students to research teams. We announced the team membership in Meeting 1. Between Meeting 2 and $3\left(\mathrm{~T}_{1}\right)$, we collected the data of initial team conflict; between Meeting 7 and $8\left(T_{2}\right)$, we measured midpoint team conflict; between Meeting 12 and $13\left(\mathrm{~T}_{3}\right)$, we collected the data of final team conflict and team satisfaction. Team performance, measured by proposal grades, was collected six weeks after the end of the course.

One hundred and twenty three participants were randomly assigned into 43 teams, including thirty-five three-person teams, seven two-person teams, and one four-person team (one person joined the course in Meeting 2). All participants completed the team conflict questionnaire at $\mathrm{T}_{1}$. At $\mathrm{T}_{2}$, missing were the data from eight persons in seven three-person teams, two persons in one two-person team and one person in the four-person team. Moreover, between $T_{1}$ and $T_{2}$. The student who joined the four-person team latest dropped out the course. At $\mathrm{T}_{3}$, missing were the data from fifteen persons in thirteen three-person teams, two persons in one twoperson team and one person from the four-person team. We excluded the twoperson team with both missing members at $T_{2}$ and $T_{3}$ from further analyses. In preliminary analyses, we examined the randomness of missing data and the equivalence of all included variables between complete teams and teams with missing members, and between three-person teams and two-person teams.

The average age of the initial 123 course participants was 24 years (s.d. = 1.55). $64.2 \%$ of them were males. The participants were of 20 different nationalities- $35.8 \%$ Dutch, 35\% German, $4.1 \%$ Belgian, and 4.1\% Italian. $38.2 \%$ of them had worked full-time before the course; $44.7 \%$ of them majored in strategy and

\footnotetext{
${ }^{27}$ See Footnote No.12 (p.28).
} 
innovation, $24.4 \%$ in entrepreneurship, $17.1 \%$ in organization, and the others in information management, marketing and so forth.

\section{Measures}

Team conflict. Team conflict was measured by Jehn and Mannix's (2001) nine-item scale of team task, relationship, and process conflict. We adapted the five-Likert-point scale to the particular research setting by replacing "work group" in the original scale with "research team" and emphasizing the assessment of the items "at this moment." Task conflict items include, for example, "How frequently do you have disagreements within your team about the research proposal task you are working on?" Process conflict items include, for example, "How often are there disagreements about who should do what in your research team?" Relationship conflict items include, for example, "How much relationship tension is there in your research team?" The administered scale is presented in Appendix A.

We calculated Cronbach's alpha for each conflict type at each time moment to test the interval validity of the scales. Cronbach's alpha of task conflict is $.83, .88$, and .86 at $\mathrm{T}_{1}, \mathrm{~T}_{2}$, and $\mathrm{T}_{3}$, respectively. Cronbach's alpha of relationship conflict is $.72, .81$, and .91 at $\mathrm{T}_{1}, \mathrm{~T}_{2}$, and $\mathrm{T}_{3}$, respectively. Cronbach's alpha of process conflict is $.78, .83$, and .91 at $\mathrm{T}_{1}, \mathrm{~T}_{2}$, and $\mathrm{T}_{3}$, respectively. These results justify the aggregation of the item-level scores into composite scores.

We then calculated the $r_{w g}$ for each conflict type at each time moment to examine the interrater agreement (IRA). For task conflict, the average $r_{w g}$ across the 42 teams in the sample is $.74, .75$, and .77 at $T_{1}, T_{2}$, and $T_{3}$, respectively. The average $r_{w g}$ for relationship conflict is $.80, .83$, and .66 at $T_{1}, T_{2}$, and $T_{3}$, respectively. The average $r_{w g}$ for process conflict is $.82, .78$, and .70 at $T_{1}, T_{2}$, and $T_{3}$, respectively. As LeBreton and Senter (2008) suggest, an IRA value between .71 and .90 indicates strong agreement among raters toward the rated targets, in this case, among individual team members towards their teams, and an IRA value between .51 and .70 indicates moderate agreement among raters. These results justify the aggregation of individual-level scores to the team level.

Team performance. Team performance was measured by the original proposal grades (on the scale from 1 to 10) standardized across the three tutors of the course, given that each tutor graded the proposals of their own students. Although the proposal grades were based on the same set of evaluation criteria (see Appendix B), the tutor effect might be present due to the three tutors' idiosyncratic standards concerning the proposal quality. We then took into account the means and standard deviations (S.D.) of the three tutors' grades and calculated the standardized proposal grades by the following function.

Standardized grade $=\left[\left(\text { Original grade }- \text { Mean grade }{ }_{\text {tutor }}\right) / \text { S.D. of grade } \text { tutor }\right]^{*}$ S.D. of grade course + Mean grade course 
By this correction, an original proposal grade of 8 from the strictest tutor (Mean ${ }_{\text {Tutor } 2}=6.97$, S.D. Tutor2 $=0.90$ ) was adjusted to 8.65 , whereas an 8 from the least strict tutor $\left(\right.$ Mean $_{\text {Tutorl }}=7.81$, S.D. Tutorl $=1.21$ ) was adjusted to 7.51, as if all the proposals would have been graded by one tutor with coherent quality standard of the proposals.

Team satisfaction. Team satisfaction was measured by Behfar and colleagues' (2008) five-item team satisfaction scale, adapted to the particular research setting. We recalibrated the original nine-Likert-point scale into a five-Likert-point and asked participants to what extent they agreed with the items that "I am satisfied with working in my research team," "I would like to work with my team members on other team assignments in the future if given the opportunities to do so," "I really like my team members," "My team members and I have become friends after working together on the IBR [International Business Research] team assignment," and "Overall our research team is doing a good job." The administered scale is presented in Appendix A. Cronbach's alpha of the team satisfaction scale yields a reliability coefficient of .89 and justifies the aggregation of the item-level scores to one composite score. The average $r_{w g}$ across the 42 teams in the sample for the composite team satisfaction score yields a value of .77 and suggests strong agreement of individual team members in their evaluation of team satisfaction. It justifies the aggregation of individually perceived team satisfaction to the team level.

\section{Analyses}

In the preliminary analysis, we tested whether teams with and without missing members at $T_{2}$ and $T_{3}$ respectively differed in the initial (i.e. $T_{1}$ ) level of task, process, and relationship conflict and whether they differed in the midpoint (i.e. $T_{2}$ ) and end (i.e. $T_{3}$ ) level of task, process, and relationship conflict, team performance, and team satisfaction. We also tested whether teams of different initial size (i.e. twoperson, three-person, versus four-person) differed in all the aforementioned variables. We performed the Analysis of Variance (ANOVA) for the preliminary analysis. Non-significant results would indicate no impact of missing data and team size on the included variables.

To test Hypothesis 1a, 2a, and 3a, we followed both the intra-subject and inter-subject approach. Within the intra-subject approach, we first calculated, for each conflict type, the $Z$-scores of each team across the three time moments. The $Z$ scores capture the information on conflict dynamics over time regardless of conflict level. We then performed hierarchical clustering analysis to identify the present conflict dynamics patterns. We chose cosine distance and within-cluster similarity maximization as the clustering method, which clustered teams of similar $Z$-score "structures" (i.e. the dynamics patterns) rather than $Z$-score levels. With the intersubject approach, we performed repeated-measures ANOVA to examine the withinsubjects effect of task, relationship, and process conflict. 
To test Hypothesis $1 \mathrm{~b}$ and $1 \mathrm{c}, 2 \mathrm{~b}$ and $2 \mathrm{c}$, and $3 \mathrm{~b}$ and $3 \mathrm{c}$, we also followed both approaches. With the intra-subject approach, we applied the same method as above to identify clusters of teams with similar dynamic patterns and then examined whether the cluster membership would lead to systematic differences in team performance and team satisfaction. We entered the categorical variable that indicated each team's cluster membership as a between-subjects factor in ANOVA to examine the impact of conflict dynamics on team performance and team satisfaction. Although linear regression with dummy variables would have been more suitable for testing the hypotheses, we did not apply this because of the small sample size $(\mathrm{n}=42)$. With the inter-subject approach, we followed the procedure in Jehn and Mannix's (2001) study. We first split teams into a high-performing group $(n=17)$ and a low-performing group $(n=25)$ by the median of the standardized proposal grades (i.e. 7.51 on a 1-to-10 scale) and then performed repeated-measures MANOVA to test whether high- and low-performing teams differ in the level of team task, relationship, and process conflict at the three time moments (Hypothesis $1 \mathrm{~b}, 2 \mathrm{~b}$, and $3 \mathrm{~b})$. Likewise, we examined whether high-satisfaction teams $(n=23)$ and low-satisfaction teams $(n=19)$, split by the median of team satisfaction (i.e. 3.33 on a 1-to-5 scale), differ in the level of team task, relationship, and process conflict at the three time moments (Hypothesis 1c, 2c, and 3c).

\section{Results}

Results from the preliminary analysis suggest that team member missing at $T_{2}$ and $\mathrm{T}_{3}$ respectively did not relate to the initial, midpoint, and end level of task, relationship, and process conflict. Neither did it relate to team performance or team satisfaction. The initial team size did not relate to the initial, midpoint, and end level of each conflict type or team performance, but it did affect team satisfaction $\left(\mathrm{F}_{(2}\right.$, ${ }_{42}=3.48, \mathrm{p}<.05$ ). In particular, the six two-person teams reported significantly lower satisfaction than the 35 three-person teams $\operatorname{did}(\mathrm{p}<.05)$, that is, 2.45 versus 3.33 on the 1-to-5 scale. Therefore, the initial time size was controlled for in the analyses of team satisfaction. The descriptive statistics and correlations of the included variables are reported in Table 5.1. 


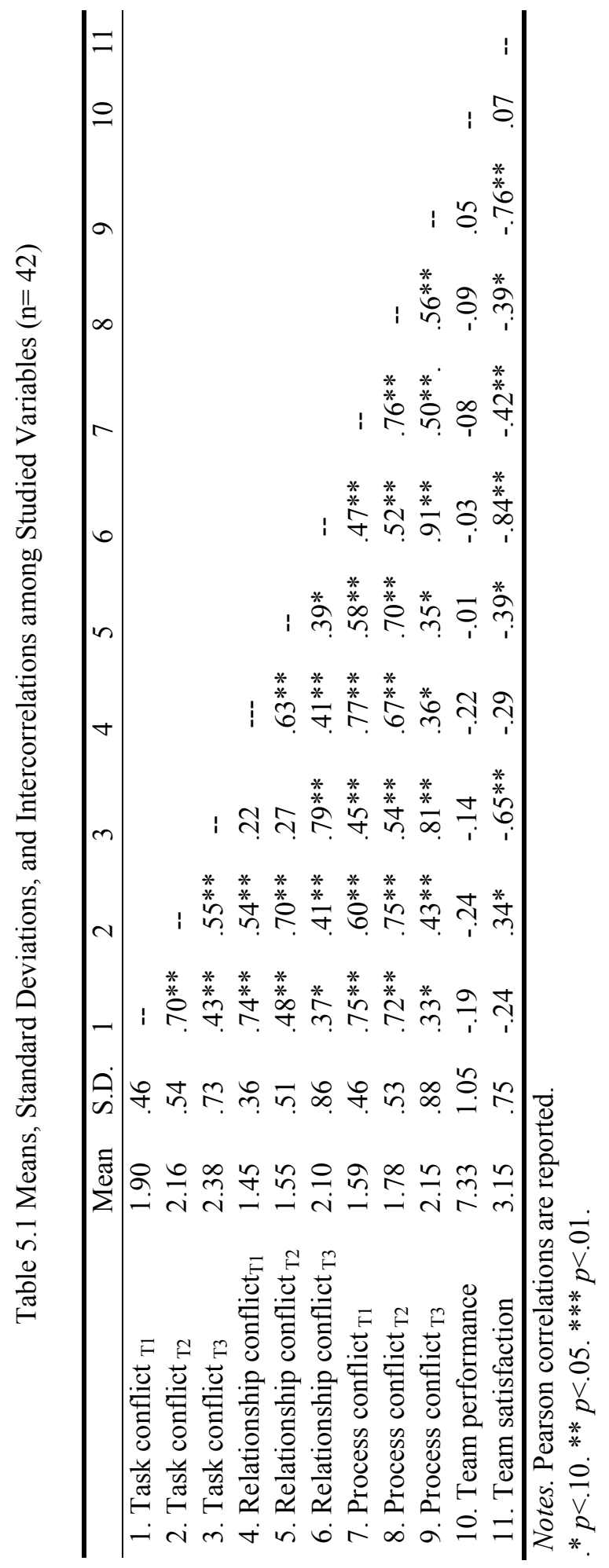


Conflict level over time. Results from the intra-subject approach suggest that for task conflict, six teams out of the total $42(14 \%)$ demonstrate the decelerated increase pattern over time, but most teams, that is, ten teams (24\%) demonstrate the accelerated increase pattern. For relationship conflict, most teams, that is, 12 teams (29\%) demonstrate the accelerated increase pattern, as predicted. For process conflict, four teams $(10 \%)$ demonstrate the decelerated increase pattern over time, but most teams, that is, 11 teams $(26 \%)$ demonstrated the accelerated increase pattern. In total, the 42 teams demonstrated 12 out of the total 17 temporal dynamics patterns for task conflict, 13 for process conflict, and 12 for relationship conflict. Hypothesis 2a is partially supported; Hypothesis 1a or 3a are not supported. We summarize the emergent conflict dynamics patterns and the distribution of the 42 teams across the patterns in Table 5.2. Moreover, with the five-category scheme, we find that in 21 teams task conflict continues to increases throughout team projects; 11 teams demonstrate the inverted-U-shape pattern; seven teams demonstrate the Ushape pattern; in two teams task conflict continues to decrease over time; one team's task conflict remains stable over time. In 20 teams' relationship conflict continues to increases throughout team projects; seven teams demonstrate the inverted-U-shape pattern; 11 teams demonstrate the U-shape pattern; four teams' relationship conflict continues to decrease over time; no team's task conflict remains stable over time. Twenty-two teams' process conflict continues to increases throughout team projects; eight teams demonstrate the inverted-U-shape pattern; eight teams demonstrate the U-shape pattern; four teams' process conflict continues to decrease over time; no team's process conflict remains stable over time. Moreover, with the more fine-grained 17-pattern scheme, we find that the 42 project teams demonstrate 12 dynamics patterns for task conflict, 12 patterns for relationship conflict, and 13 patterns for process conflict. 


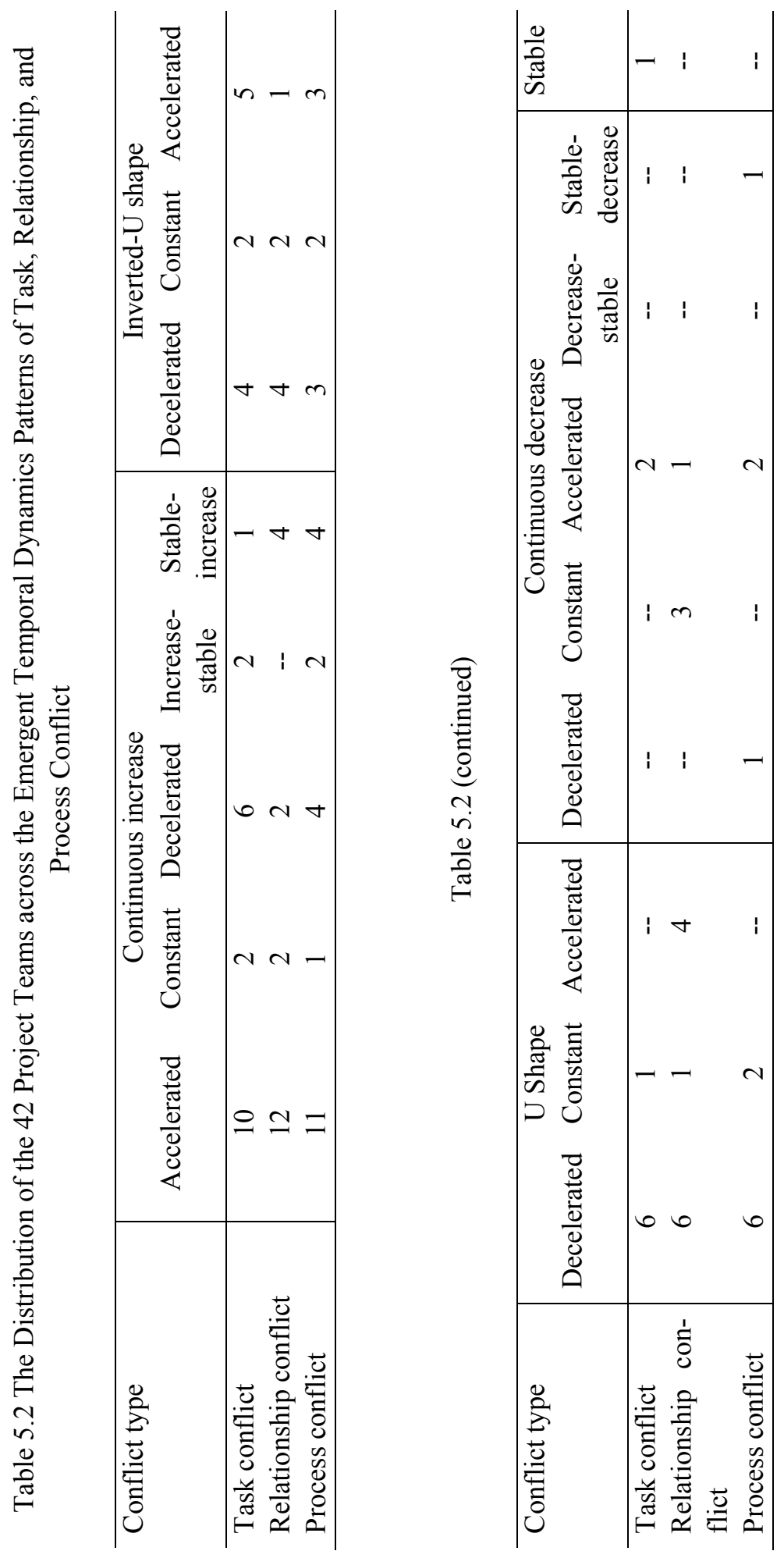


Applying the inter-subject approach, we found that the Mauchly's sphericity test from the repeated-measures ANOVA indicated a violation of the sphericity assumption for task conflict (TC), relationship conflict (RC), and process conflict $(\mathrm{PC})\left(\chi_{\mathrm{TC}(2)}^{2}=13.99, p<.01 ; \chi_{\mathrm{RC}(2)}^{2}=23.14, p<.01 ; \chi_{\mathrm{PC}(2)}^{2}=28.07, p<.01\right)$. We then used within-subjects contrasts to correct such violation. For task conflict, the within-subjects contrast shows a linear increase across the three time moments $\left(\mathrm{F}_{(1}\right.$, $\left.{ }_{42}=21.25, p<.01\right)$. Pairwise comparison reveals that team task conflict level increases from 1.90 to 2.16 between $\mathrm{T}_{1}$ and $\mathrm{T}_{2}(p<.05)$, and from 2.16 to 2.39 between $\mathrm{T}_{2}$ and $\mathrm{T}_{3}(p<.05)$. The between-subject effect is also significant $\left(\mathrm{F}_{(1,42)}=818.59\right.$, $p<.01$ ), indicating diversity in teams' development of task conflict over time. For relationship conflict, the within-subjects contrast shows a quadratic increase over time $\left(\mathrm{F}_{(1,42)}=8.74, p<.01\right)$. Pairwise comparison reveals that relationship conflict level did not differ between $T_{1}$ and $T_{2}$ but increased from 1.55 to 2.10 between $T_{2}$ and $\mathrm{T}_{3}(p<.05)$. The between-subjects effect is significant as well $\left(\mathrm{F}_{(1,42)}=559.28\right.$, $p<.01$ ), indicating substantial variety in dynamic patterns between the teams. For process conflict, the within-subjects contrast shows a linear increase over time $\left(\mathrm{F}_{(1}\right.$, ${ }_{42}=22.05, p<.01$ ). Pairwise comparison points at an increase from 1.60 to 1.78 between $\mathrm{T}_{1}$ and $\mathrm{T}_{2}(p<.05)$ and from 1.78 to 2.15 between $\mathrm{T}_{2}$ and $\mathrm{T}_{3}(p<.05)$. Once again, we find evidence of inter-team variety in conflict dynamics $\left(\mathrm{F}_{(1,42)}=506.39\right.$, $p<.01$ ). These findings of the inter-subject approach also provide partial support for Hypothesis 2a but no support for Hypothesis 1a and 3a. We summarize the results in Table 5.3 and graph the sample means of the three conflict types across the three moments in Figure 5.1a and individual teams' task conflict dynamics patterns in Figure 5.1b.

Table 5.3 The Repeated-Measures MANOVA Results of Team Task, Relationship, and Process Conflict over Time $(n=42)$

\begin{tabular}{lcccccc}
\hline \multicolumn{1}{c}{ Effects } & Task conflict & \multicolumn{2}{c}{ Relationship conflict } & Process conflict \\
& & \multicolumn{1}{c}{} & & & & \\
\cline { 2 - 7 } & $F$ & $d f$ & $F$ & $d f$ & $F$ & $d f$ \\
\hline Between-subjects effect & $818.59^{* * *}$ & 1 & $559.28^{* * *}$ & 1 & $506.39^{* * *}$ & 1 \\
& & & & & & \\
Within-subjects contrast & & & & & & \\
Linear & $21.25^{* *}$ & 1 & $27.23^{* *}$ & 1 & $22.05^{* *}$ & 1 \\
Quadratic & .09 & 1 & $8.74^{* *}$ & 1 & 2.10 & 1 \\
\hline Note. ${ }^{* *} p<.05 .{ }^{* * *} p<.01$. & & & & & &
\end{tabular}


Figure 5.1a The Sample-Level Development Patterns of Task, Relationship and Process Conflict over Time $(n=42)$

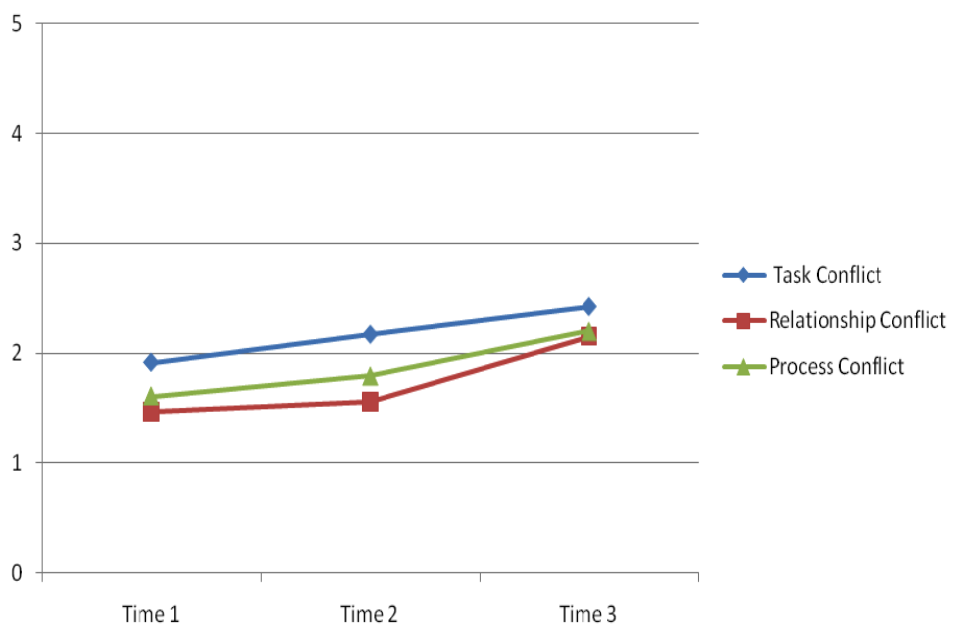


Figure 5.1b Individual Teams' Development Patterns of Team Task Conflict over Time $(n=42)$

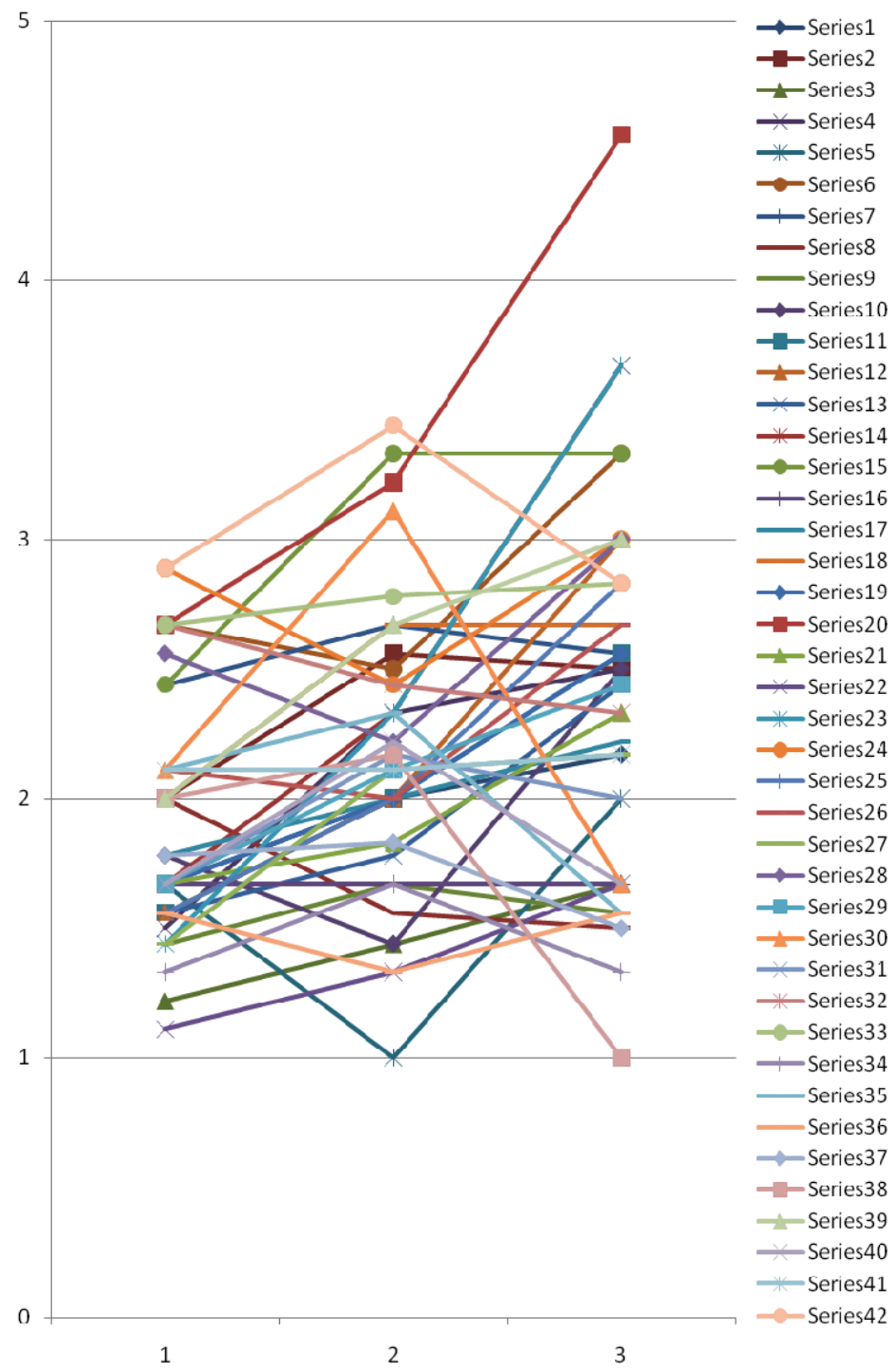

The impact of team conflict dynamics over time on team performance. Results from the intra-subject approach do not show any significant overall effect of the categorical variables comprising the dynamic patterns of task, relationship and process conflict on performance. The results for the inter-subject approach are similar: We find no significant effect of the performance when using high and low 
performance (based on a median split) as a between-subjects factor. Therefore, we must conclude that neither approach provides support for Hypothesis $1 b, 2 b$, or $3 b$. Team conflict dynamics over time does not affect team performance.

The impact of team conflict dynamics over time on team satisfaction. The intra-subject approach shows a relationship between task conflict dynamics and team satisfaction. Teams with the inverted-U shape pattern reported significantly higher team satisfaction at the end than the other teams $\left(\mathrm{F}_{(1,42)}=5.99, \mathrm{p}<.01\right)$, that is, $\mathrm{M}_{\mathrm{TS}}=$ 3.53 on the 1-to- 5 scale in comparison to $\mathrm{M}_{\mathrm{TS}}=3.02$. We consider Hypothesis $1 \mathrm{c}$ to be supported.

Moreover, we found, for task conflict, significant overall difference in team satisfaction level across the five categories $\left(\mathrm{F}_{(4,42)}=2.56, \mathrm{p}<.10\right)$ but no significant difference between the baseline teams and any other cluster of teams. Pairwise comparison results suggest that teams with the inverted $\mathrm{U}$ shape patterns $\left(\mathrm{M}_{\mathrm{TS}}=\right.$ 3.57) and continuous decrease patterns $\left(\mathrm{M}_{\mathrm{TS}}=3.90\right)$ of task conflict reported significantly higher team satisfaction level than teams with the U-shape patterns $\left(\mathrm{M}_{\mathrm{TS}}\right.$ $=2.96)$ and the continuous increase patterns $\left(\mathrm{M}_{\mathrm{TS}}=2.90\right)$, respectively ${ }^{28}$. As long as the task conflict of a project team decreases in the second half time of the project, regardless of its development pattern in the first half time of the project, the team, as a temporally-integral entity, will report high satisfaction level at the end of the project.

For relationship conflict, we find that teams with the inverted-U shape and $\mathrm{U}$ shape pattern had significantly higher team satisfaction than the other teams $\left(\mathrm{F}_{(1,42)}=5.16, \mathrm{p}<.01\right)$, that is, $\mathrm{M}_{\mathrm{TS}}=3.43$ in comparison to 2.94 . Therefore, we considered Hypothesis $2 \mathrm{c}$ to be supported. Using the broader categorization based on change direction, we find a significant difference of team satisfaction level across the five categories $\left(\mathrm{F}_{(3,42)}=5.08, \mathrm{p}<.01\right)$. Moreover, pairwise comparison suggests that teams with the inverted $\mathrm{U}$ shape patterns $\left(\mathrm{M}_{\mathrm{TS}}=3.51, \mathrm{t}=2.75, \mathrm{p}<.01\right)$, $\mathrm{U}$ shape patterns $\left(\mathrm{M}_{\mathrm{TS}}=3.38, \mathrm{t}=2.50, \mathrm{p}<.05\right)$, and continuous decrease patterns $\left(\mathrm{M}_{\mathrm{TS}}=3.86\right.$, $\mathrm{t}=2.97, \mathrm{p}<.01)$ of relationship conflict report significantly higher team satisfaction level than teams with the continuous increase pattern of relationship conflict (i.e. $\mathrm{M}_{\mathrm{TS}}=2.76$ ). Teams with the continuous increase patterns of relationship conflict over time are the least satisfied teams in the end. As long as the relationship conflict of a project team decreases in at least one half time of the project, it will report a high satisfaction level at the end of the project.

For process conflict, that team satisfaction level does not differ between teams with the inverted-U shape pattern and the other teams. Hypothesis $3 \mathrm{c}$ was not supported. Using the five broader categorization scheme, we found significant difference of team satisfaction level across the four emergent categories $\left(\mathrm{F}_{(3,42)}=2.62\right.$, $p<.10)$. Pairwise comparison indicates that teams with the inverted-U shape patterns (TS=3.57, $\mathrm{t}=1.97, p<.10)$, and continuous decrease patterns $\left(\mathrm{M}_{\mathrm{TS}}=3.58, \mathrm{t}=2.07\right.$,

\footnotetext{
${ }^{28} \mathrm{P}<.05$ between the inverted-U shape and $\mathrm{U}$ shape group; $\mathrm{p}<.05$ between the inverted- $\mathrm{U}$ and increase group; $\mathrm{p}<.10$ between the decrease and $\mathrm{U}$ shape group; $\mathrm{p}<.10$ between the decrease and increase group.
} 
$p<.05)$ of process conflict have significantly higher team satisfaction than teams with the $U$ shape patterns of process conflict $\left(\mathrm{M}_{\mathrm{TS}}=2.73\right)$. In other words, teams with the $U$ shape pattern are the least satisfied in the end. Process conflict decrease of a project team in the second half time of the project, regardless of its development pattern in the first half time of the project, enhance the team's satisfaction at the end of the project. We summarize the results of the intra-subject approach in Table 5.4.

Table 5.4 The ANOVA Results of the Impact of Team Task, Relationship, and Process Conflict Dynamics on Team Satisfaction $(n=42)$

\begin{tabular}{lll}
\hline Variable & Team performance & Team satisfaction $^{\text {a }}$ \\
\hline Task conflict & & \\
Increase & 7.24 & 3.01 \\
Inverted-U shape & 7.16 & 3.53 \\
U shape & 7.75 & 2.88 \\
Decrease & 7.07 & 3.98 \\
Stable & 8.56 & 2.20 \\
$F$-statistics & 0.74 & $2.56^{*}$ \\
Relationship conflict & & \\
Increase & 7.40 & 2.76 \\
Inverted-U shape & 7.41 & 3.51 \\
U shape & 7.08 & 3.38 \\
Decrease & 7.5 & 3.86 \\
$F$-statistics & 0.26 & $5.08^{* * *}$ \\
Process conflict & & \\
Increase & 7.44 & 3.07 \\
Inverted-U shape & 6.74 & 3.57 \\
U shape & 7.33 & 2.73 \\
Decrease & 7.92 & 3.58 \\
$F$-statistics & 1.37 & $2.26^{*}$ \\
\hline
\end{tabular}

Note. Team size is controlled.

$* p<.10 . * * * p<.01$.

When applying the inter-subject approach, we find differences in the patterns of team task, relationship, and process conflict over time between high-satisfaction teams and low-satisfaction teams $\left(\mathrm{F}_{\mathrm{TC}(1,42)}=12.21, p<.01 ; \mathrm{F}_{\mathrm{RC}(1,42)}=22.34, p<.01\right.$; $\left.\mathrm{F}_{\mathrm{PC}(1,42)}=13.89, p<.01\right)$. For task conflict, within-subjects contrast results show that teams' task conflict follows a first-increase-then-stable pattern across the three time moments $\left(\mathrm{F}_{(1,23)}=6.88, p<.05\right)$. Pairwise comparison results indicate that team task 
conflict level increases from 1.78 to 2.06 between $T_{1}$ and $T_{2}(p<.05)$ but does not differ between $T_{2}$ and $T_{3}$. In comparison, task conflict of the group of lowsatisfaction teams differs follows an accelerated increase pattern $\left(\mathrm{F}_{(1,19)}=4.06\right.$, $p<.10)$, increasing from 2.05 to 2.29 between $\mathrm{T}_{1}$ and $\mathrm{T}_{2}(p<.05)$ and from 2.29 to 2.88 between $\mathrm{T}_{2}$ and $\mathrm{T}_{3}(p<.01)$. Thus, Hypothesis $1 \mathrm{c}$ is not supported.

The relationship conflict of high-satisfaction teams increases linearly from $T_{1}$ to $T_{3}$, whereas that of low-satisfaction teams follows a first-stable-then-increase pattern $\left(\mathrm{F}_{(1,19)}=14.93, p<.01\right)$, being stable from $\mathrm{T}_{1}$ to $\mathrm{T}_{2}$ but increasing from 1.68 to 2.77 between $\mathrm{T}_{2}$ and $\mathrm{T}_{3}(p<.01)$. Hypothesis $2 \mathrm{c}$ is not supported.

Process conflict of high-satisfaction teams increases from 1.48 to 1.66 between $T_{1}$ and $T_{2}(p<.05)$ but remains stable between $T_{2}$ and $T_{3}$. For low-satisfaction teams, process conflict follows a accelerated increase pattern $\left(\mathrm{F}_{(1,19)}=8.00, p<.01\right)$, that is, increasing from 1.74 to 1.93 between $\mathrm{T}_{1}$ and $\mathrm{T}_{2}(p<.05)$ and from 1.93 to 2.74 between $\mathrm{T}_{2}$ and $\mathrm{T}_{3}(p<.01)$. Hypothesis $3 \mathrm{~b}$ is therefore not supported. We summarize the results in Table 5.5 and depict the temporal dynamics patterns of task, relationship, and process conflict of the high-satisfaction teams and lowsatisfaction teams in Figure 5.2a and Figure 5.2b.

Table 5.5 The Repeated-Measures MANOVA Results of the Impact of Team Task, Relationship, and Process Conflict over Time on Team Satisfaction

\begin{tabular}{|c|c|c|c|c|c|c|}
\hline \multirow[t]{2}{*}{ Effects } & \multicolumn{2}{|c|}{ Task conflict } & \multicolumn{2}{|c|}{$\begin{array}{l}\text { Relationship } \\
\text { conflict }\end{array}$} & \multicolumn{2}{|c|}{$\begin{array}{l}\text { Process con- } \\
\text { flict }\end{array}$} \\
\hline & $F$ & $d f$ & $F$ & $d f$ & $F$ & $d f$ \\
\hline Between-subjects effect $(N=42)$ & $12.21 * * *$ & 1 & $22.34 * * *$ & & $13.89 * * *$ & 1 \\
\hline $\begin{array}{l}\text { Within-subjects contrast in high- } \\
\text { satisfaction teams }(n=23)\end{array}$ & & & & & & \\
\hline Linear & $2.90 *$ & 1 & $3.31^{*}$ & 1 & 2.79 & 1 \\
\hline Quadratic & $6.88 * *$ & 1 & 0.68 & 1 & 1.86 & 1 \\
\hline $\begin{array}{l}\text { Within-subjects contrast in low- } \\
\text { satisfaction teams }(n=19)\end{array}$ & & & & & & \\
\hline Linear & $29.17 * * *$ & 1 & $50.00 * * *$ & 1 & $31.60 * * *$ & 1 \\
\hline Quadratic & $4.06^{*}$ & 1 & $14.93 * * *$ & 1 & $8.00 * *$ & 1 \\
\hline
\end{tabular}


Figure 5.2a The Development Patterns of Task, Relationship and Process Conflict over Time of Low-Satisfaction Teams $(n=19)$

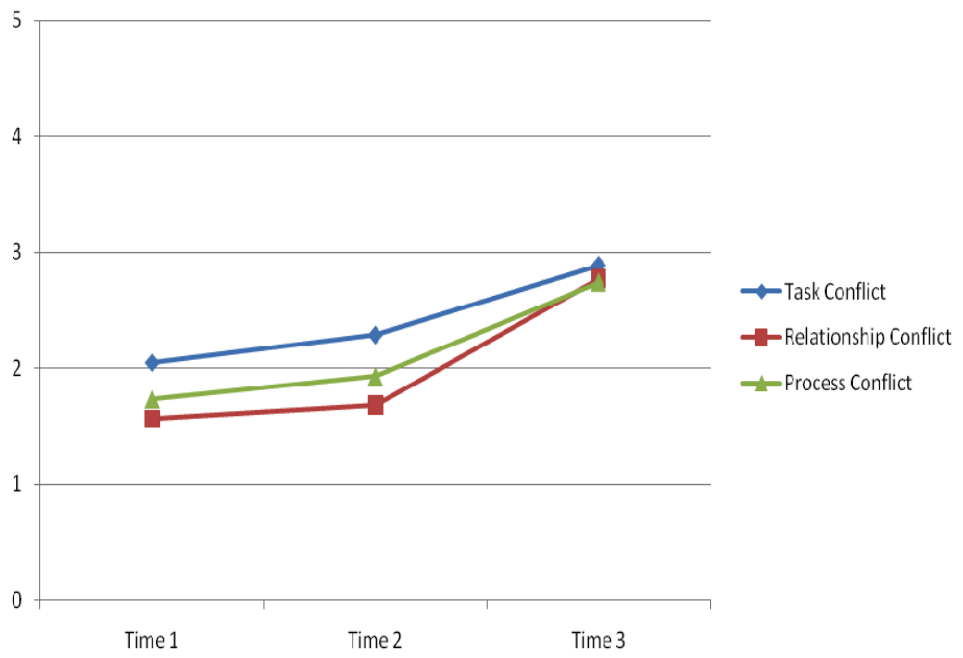

Note. The difference of team relationship conflict is not significant between Time 1 and Time 2 .

Figure 5.2b The Development Patterns of Task, Relationship and Process Conflict over Time of High-Satisfaction Teams $(n=23)$

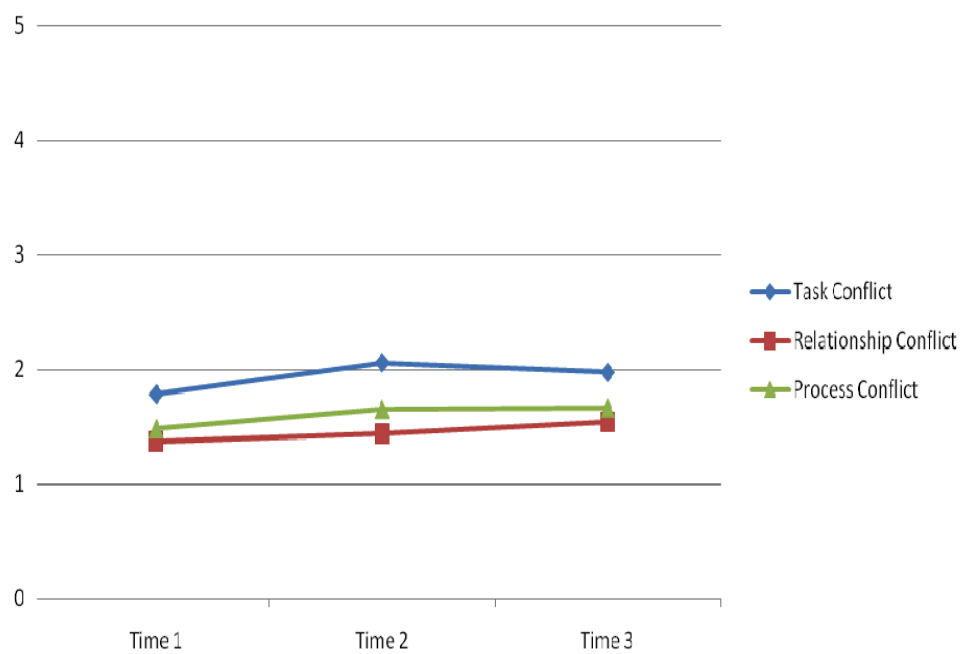


Note. The difference of team task and process conflict is not significant between Time 2 and Time 3 . The difference of team relationship conflict is not significant between Time 1 and Time 2 or between Time 2 and Time 3 .

\section{Discussion}

The aim of this chapter is to examine the development of team conflict over time and its impact on team performance and team satisfaction by contrasting results from the intra-subject longitudinal approach (i.e. dynamics-based clustering in combination with MANOVA) and the inter-subject longitudinal approach (i.e. repeated-measures MANOVA and depicting the dynamics of group means over time). We report differential findings from the two approaches, which pertain to two aspects of the punctuated equilibrium model, that is, the linearity or nonlinearity of team processes over time and the impact of heterogeneous team process dynamics on team outcomes. First, results from the intra-subject approach suggest that most project teams' task, relationship, and process conflict develop in a nonlinear fashion over time. In contrast, results from the inter-subject approach suggest that overall team task and process conflict develop linearly over time, while team relationship conflict increases at an accelerated rate over time. Second, the results from both approaches suggest that team conflict dynamic pattern over time does not affect team performance, whereas it does affect team satisfaction at the end. More specifically, it appears that the dynamic pattern of team task and process conflict in the second half of team projects affects team satisfaction. However, for relationship conflict, results from the two approaches differ. Results from the intra-subject approach show that the dynamic pattern of team relationship conflict throughout team project affects team satisfaction, whereas results from the inter-subject approach show that in high-satisfaction teams' relationship conflict increases linearly over time and in low-satisfaction teams it is stable in the first half of the team projects and then increases in the second half. In other words, the dynamic pattern of team relationship conflict in the first half of team projects affects team satisfaction at the end. We elaborate the findings and their theoretical and managerial implications as follow.

\section{Theoretical Implications}

Team conflict development over time. In most project teams, task, relationship, and process conflict develop nonlinearly over time and they do so in rather different ways. The dynamic patterns shown by the inter-subject and intra-subject longitudinal approach are remarkably different. Whereas the inter-subject approach suggests a linear increase of task and process conflict and an accelerated increase of relationship conflict over time the intra-subject approach reveals that only few teams show these patterns. Two teams (5\%) demonstrate the linear increase pattern of task con- 
flict and only one team (2\%) shows this pattern in the case of process conflict. For relationship conflict, only four teams $(10 \%)$ demonstrate a first-stable-then-increase pattern as the conclusion drawn from the inter-subject approach.

These discrepant findings highlight the different logics of the intra-subject and inter-subject longitudinal approach. In the intra-subject approach, each subject's temporal dynamic pattern of an attribute (e.g. each team's conflict dynamics pattern in the study) is considered as a holistic phenomenon and assumed to be heterogeneous across subjects. The notion of one overall sample-level pattern does not exist. Instead, the sample-level "pattern" consists of the number of emergent patterns or pattern categories and their distribution across teams in the sample. In the intersubject approach, each subject's pattern is considered as a random variation of "one overall sample-level pattern". Therefore, the "sample-level pattern" is first estimated and subjects' patterns are considered as either all following the "samplelevel" pattern or deviating from it randomly. Our findings invite careful interpretations of the results from the inter-subject approach, because the estimated "samplelevel pattern" can be an aggregate pattern that consolidate various convergent and divergent individual patterns, instead of a pattern that consist of only homogeneous individual patterns.

Furthermore, as the intra-subject approach suggests, in most project teams conflict develops nonlinearly over time and that the linear increase, decrease, and stable patterns are rare. Team conflict, a specific team process elicited by the accomplishment of team tasks, develops nonlinearly over time, as predicted by the punctuated equilibrium model (Gersick, 1988, 1989, 1991). Over time, project teams alternate between two transition stages and two action stages (Ford \& Sullivan, 2004; Gersick, 1988, 1991; Marks et al., 2001; Waller, Zellmer-Bruhn, \& Giambatista, 2002). In the two transition stages (i.e. the beginning and during a team project), teams set goals, formulate strategies, and make plans for the upcoming taskwork and team functioning. In the two action stages (i.e. before and after the second team transition), teams conceptualize and implement project ideas by coordinating inside and outside the teams and monitor the progress of taskwork. As the nature and intensity of the taskwork changes throughout the four stages, the intensity of particular team interactions may change as well. In other words, not all team processes would be present or present to the same degree in a team over time (Marks et al., 2001). Team processes, including the primary task accomplishment and other elicited team processes, may develop linearly within a transition or action stage but vary nonlinearly as teams alternate through transition and action stages.

Heterogeneous team conflict dynamics over time may originate from the differential timing of the second team transition, that is, before, at, or after the exact middle time point of team projects. At the midpoint, a project team can be in the first action stage (i.e. the post-midpoint transition teams), the second transition stage (i.e. the at-midpoint transition teams), or the second action stage (i.e. the antemidpoint transition teams), whereas they are all in the first transition stage at the 
beginning of team projects and in the second action stage at the end of team projects. At the middle time point, the differential nature and intensity of team subtasks may produce differences in the intensity of team discussion and contemplation of task content and procedures, which may result in different levels of team task and process conflict. The differential timing of the second transition indicates how project teams allocate the limited temporal resources between task accomplishment and social aspects in the first and second half of team projects. In combination with the increasing work intensity over time, different time allocation between tasks and social activities may lead to different pattern of relationship conflict over time.

The impact of team conflict dynamics on team performance. We found that team performance is not associated with team conflict over time in either approach. Given the evidence in the literature that team conflict level is negatively associated with team performance (De Dreu \& Weingart, 2003; De Wit \& Greer, 2008), and that overall, high- and low-performing teams diverge in conflict development patterns over time (Jehn \& Mannix, 2001), the finding in our study is rather unexpected. We ran additional regressions of team performance on team task, relationship, and process conflict at respective time moments and found a negative relationship of team task conflict at the midpoint and end of the project with team performance, and a positive relationship of team process conflict at the end with team performance $^{29}$. In comparison, we found a weaker influence of team conflict development on team performance than of team conflict level. Two explanations may be plausible for the weak relationships between team conflict development and level and team performance: One is that the variance of the performance measure is relatively low; the other is that the performance measure (i.e. the proposal grade) accounted for only $30 \%$ the total grade of the students and might not be motivating enough for the teams to perform to their full potential. Further research is needed to investigate the relationship between team conflict dynamics and team performance.

The impact of team conflict dynamics on team satisfaction. The development pattern of team conflict over time relates to team satisfaction level at the end. For task and process conflict, the results of the inter- and intra-subject longitudinal approach are similar, and show that the late development (i.e. in the second half of team projects) of team task and process conflict affects team satisfaction at the end. For relationship conflict, the findings diverge. The intra-subject analysis shows that the development pattern throughout the entire project influences team satisfaction at the end. Teams with continuously increasing relationship conflict have lower team satisfaction than the other teams, indicating that as long as relationship conflict decreases in a team, regardless of when, such decrease will enhance team satisfaction. Results from the inter-subject approach, on the contrary, suggest that highsatisfaction teams are characterized by linearly increased relationship conflict pattern over time and low-satisfaction teams are characterized by the first-stable-then-

\footnotetext{
${ }^{29}$ The unstandardized $\beta$ coefficients are reported here. $\beta_{T C, T 2}=-0.97, \mathrm{p}<.10 ; \beta_{T C, T 3}=-0.68, \mathrm{p}<.10$; $\beta_{P C, T 3}=0.81, \mathrm{p}<.10$
} 
increase relationship conflict pattern over time. In other words, the early development of relationship conflict (i.e. increase versus stable in the first half of team projects) seems to affect team satisfaction at the end.

As discussed above, a conflict pattern found by the inter-subject approach is an aggregate pattern that consolidates various convergent and divergent individual patterns. Our results show that one should be cautious in concluding that all high- or low-satisfaction teams follow the same conflict dynamic pattern and, in this particular case, that the late development of team task and process conflict and the early development of team relationship conflict affect team satisfaction. The acknowledgement of heterogeneity in teams' conflict dynamics over time aligns with both the empirical reality that individual teams do differ in conflict dynamics and the theoretical conjecture that heterogeneous team conflict dynamics patterns leads to heterogeneous team satisfaction levels. We are inclined to base our conclusion on the results from the intra-subject approach, suggesting that it is the development of team relationship conflict throughout the entire team project period, rather than that in the first half of this period, that affects team satisfaction at the end.

The differential results imply that the intra-subject approach and inter-subject approach address distinct theoretical perspectives regarding the causal links between team conflict and team satisfaction. The intra-subject approach follows a temporally-forward logic and first examines individual teams' conflict dynamics patterns over time as a whole and next their associations with team satisfaction levels. This logic answers whether project teams with differential conflict dynamic patterns over time also differ in team satisfaction levels. In comparison, the intersubject approach follows a temporally-backward logic and first examines the associations between team conflict levels at discrete time moments and team satisfaction levels and next delineates an overall "development pattern" at the level of the sample or subsample. This logic answers whether project teams with different team satisfaction levels have differed in team conflict level at the three time moments. The differential findings suggest that the temporal dynamics and the level of team task, relationship, and process conflict at discrete time moments of team projects relate to team satisfaction in a distinct fashion.

Organization theorists' have recently called for using both dynamic processbased models and static variances-based models in studying organizational phenomena (McGrath \& Tschan, 2004; Roe, 2009; Van de Ven, 2007). Research should analyze both "within-subject variability" and "between-subject heterogeneity". In team research based on the Input-Process-Output (IPO) framework, researchers have argued for conceptualizing team processes as dynamic phenomena and concepts over time and repeatedly measuring them (Hackman, 1987; Ilgen et al., 2005; Marks et al., 2001; McGrath, 1984). In this study, we have applied the intra-subject and inter-subject approach to the same research phenomenon and found that the intra-subject approach, with its emphasis on the dynamics of team processes, does indeed offer insights that the conventional inter-subject approach 
with its emphasis on between-subject differences fails to provide. We believe that this helps to gain a more comprehensive knowledge of team processes.

\section{Managerial Implications}

This study has two major managerial implications. First, project teams and their supervisors may need to monitor the development pattern of teams' task, relationship, and process conflict over time, in order to achieve desirable team satisfaction. Teams can use various conflict resolution tactics, such as assigning individual tasks according to expertise and focusing on the content of interpersonal interactions (Behfar et al., 2008) to lower team task and process conflict over the second half of team projects and to lower team relationship conflict at any time moment. By doing so, teams will obtain high team satisfaction at the end of team projects and benefit future team collaboration. Second, the management of project teams may need to be aware that project teams' development is a highly idiosyncratic process and that teams differ largely from each other in their internal and external interactions over time. Hence, it is crucial for project teams and their management to identify the idiosyncratic development pattern of each project team and carefully apply the general knowledge of project team development learned from most other teams.

\section{Limitations}

There are four major limitations of the study. First, the sample of project teams of business graduate students may limit the generalizability of the reported findings to other types of project teams, despite the "versatility" of punctuated equilibrium theory (Van de Ven, 2007). Second, team conflict and team satisfaction were measured by self-administered questionnaires. Therefore, common method bias could potentially explain part of the reported impact of team conflict dynamics over time on team satisfaction and its lack of impact on team performance measured objectively. Third, the dependent variables performance and satisfaction were measured post hoc at a single point in time. From a temporal perspective it would have made more sense to consider performance and satisfaction as dynamic phenomena and to measure them as they unfold over time (Roe, 2008). We recommend that future research will take repeated measures of performance and analyze the dynamic relations between conflict, performance and satisfaction. Fourth, the punctuated equilibrium model provides a theoretical foundation to expect the nonlinear development of team conflict over time as well as heterogeneous conflict dynamics pattern across teams. However, this model gives a limited account of all factors operating in a working team. Although task accomplishment -- the primary goal and activity of project teams -- may substantially affect team conflict dynamics, other team interactions or processes, especially team conflict management activities may also have an influence, and explain the existence of other non-hypothesized team conflict patterns, such as the linear decrease or stable pattern. We did not investigate 
such factors, nor did we explore possible antecedents of the heterogeneous team conflict dynamic patterns. Team demographic composition or other boundary conditions of midpoint transition (e.g. team member familiarity, Okhuysen \& Waller, 2002) may help to explain the distribution of the emergent divergent team conflict development patterns, which needs to be investigated in future research 



\section{Chapter 6}

\section{Discussion and Conclusions}

In the concluding chapter, we review the findings from the previous chapters and discuss the implications for future team research. Using more advanced methods to assess group-level temporal dynamics, we discovered that most temporal characteristics are not sufficiently stable over time or across situations to predict team processes and team outputs. This finding does not only prove the wisdom of conducting stability tests preliminarily, but also suggests team researchers to study the temporal dynamics of individual temporal characteristics in its own right, rather than regarding them as personality traits and employing single moment measures. Moreover, using the intra-subject longitudinal approach to examine intra-subject temporal dynamics, we found remarkable discrepancies between temporal dynamics of conflict in a sample of teams and in individual teams. We also found a unique impact of intra-team conflict dynamics over time on team satisfaction, unrelated to the impact of team conflict level at different time moments. These findings suggest a potential theoretical contribution of using the intra-subject longitudinal approach, in addition to the conventional inter-subject longitudinal approach, in studying the temporal dynamics of an attribute.

We argue that team research can be further advanced, if the temporal dynamics of team processes are incorporated in theories and research models as a holistic concept, and if the examination of temporal dynamics is extended from team processes to team inputs and team outputs. We contend that the methods used and developed in this dissertation are, rather than limited to team research, applicable to research on individual behaviors as well. We conclude the chapter with a discussion of managerial implications and limitations of the dissertation. 
The lack of convincing support for team effectiveness models developed within the Input-Process-Output (IPO) framework has prompted team researchers to look for improvements in two directions. The first direction is to broaden the notion of team inputs from surface-level demographic features to deep-level psychological attributes. The argument goes that in addition to visible team composition (e.g. demographics), unobservable team composition (e.g. beliefs, values, personality traits) explains inter-team differences in team performance and social outputs (Harrison, Price, \& Bell, 1998; Harrison, Price, Gavin, \& Florey, 2002). The second direction is to unpack the "black box" of team processes between team inputs and team outputs and examine what teams do to transform team inputs into team outputs (Ancona \& Caldwell, 1992; Jehn, Northcraft, \& Neale, 1999; Lawrence, 1997; Pelled, 1996; Pelled, Eisenhardt, \& Xin, 1999).

Unfortunately, empirical studies following the two research directions have achieved rather limited success, so far. For example, in a recent meta-analysis, Bell (2007) concludes that the predictions of team performance from team deep-level psychological attributes are rather inconsistent. Likewise, team process research presents inconsistent findings of team processes' intermediary (i.e. mediating and moderating) effects on the relationship between team inputs and team outputs. For example, De Dreu and Weingart (2003) have noted that some studies report positive effect of team task conflict on team performance (e.g. Jehn, 1994) whereas some others show the negative effect (e.g. Barsade, Ward, Turner, \& Sonnenfeld, 2000).

Focusing on the temporal dimension of team research, this dissertation offers a perspective to understand the limited success of recent research on deep-level psychological characteristics and team processes and to build better models in the future. We pointed out that in order for the IPO-framework-based research to be effective, two temporal conditions must be fulfilled. The first is that deep-level psychological attributes, such as time urgency and polychronicity, are stable over time and across situations, so that they can generate valid and reliable predictions of team processes (i.e. what happens in teams) and team outputs (i.e. what is produced by teams). Low intertemporal and/or intersituational stability of deep-level psychological attributes may explain poor and inconsistent predictions of team performance, in addition to other contingent factors, such as research purpose and team type between laboratory research and field research (Bell, 2007). The second condition is that teams differ in their process dynamics over time, so that inter-team differences in intra-team process dynamics over time can be associated with inter-team differences in team input levels and/or team output levels. We have argued that team processes, defined as team internal and external interactions over time (Marks et al., 2001), should be conceptualized and empirically investigated as a holistic phenomenon that is undividable by discrete time moments within a team. 


\section{Review of Our Findings}

In Chapters 2 and 3, we presented two studies in which the first condition was empirically examined. Since the test-retest reliability coefficient, the most commonly used method to assess temporal stability, was suggested to be inadequate, we opted for a more sophisticated methodology that allows to investigate alpha, beta, and gamma change (Golembiewski, Billingsley, \& Yeager, 1976) with a latent growth modeling (LGM) procedure developed by Chan (1998) and Lance, Vandenberg \& Self, (2000). In Chapter 2, we studied time urgency, polychronicity, time perspective, and temporal depth over six months and found that the measurement of most temporal characteristics varies over time (i.e. gamma change occur). In Chapter 3 we used a quasi-experimental design and found an effect of a computer simulation designated to influence subjects' scores on some dimensions of time urgency and time perspective.

These findings demonstrate that most temporal characteristics are, indeed, not sufficiently stable over time and across situations to predict team processes and team outcomes. Their instability raises two concerns, namely reverse causality and time- or situation-contingent predictiveness. If a temporal characteristic is not stable, it might not influence a particular team process or output, but rather be influenced by it. For example, team members' working speed - a time urgency component - is theorized to influence how likely project teams meet project deadlines. Given the low intertemporal and intersituational stability found in our studies, it is possible that project teams' working speed result from the exposure to intermediary temporal milestones and final deadlines. The second concern relates to the possibility that the power to predict team processes and team outputs is contingent on when and where the predictor is measured. It is necessary to preliminarily examine the intertemporal and intersituational stability of team deep-level characteristics via the alpha-beta-gamma-change typology and latent growth modeling.

Our first two studies prove the wisdom of conducting preliminary stability tests. Without such tests, researchers may gather snapshot information of intrinsically dynamic researched phenomena (i.e. temporal characteristics in this dissertation) and thereby engender the risk to report inconsistent relationships with theorized antecedents and consequences. These findings lead us to advise team researchers to abstain from using single moment measures of temporal characteristics in pertinent empirical studies. The evidence on the intertemporal stability of time urgency, polychronicity, and time perspective in Chapter 2 suggests the prudence in using single measures of these temporal characteristics. The fact that the construct validity and calibration changed over a period of six months underlines the necessity to assess the temporal equivalence of the measurement scales.

Chapter 4 and 5 were devoted to examining the second temporal condition, that is, teams should differ in their process dynamics over time. The existence of such inter-team differences is a necessary condition to establish causal relationships 
of team processes with team inputs and team outputs. The examination of the statistical relations among team inputs, team processes, and team outputs requires the measurement of all included constructs across teams. Thus, team processeses must be conceptualized and measured as temporal dynamic patterns that characterize individual teams. Although the dynamic and idiosyncratic nature of team processes has been occasionally documented in extant literature (Chang, Bordia, \& Duck, 2003; Ericksen \& Dyer, 2004; Gersick, 1989), the assessment of inter-team differences in intra-team dynamic patterns over time is exactly what has been missing in $I P O$-based team research. When studying changes over time, team researchers have typically used research methods that assume a group-level dynamic pattern to by default apply to the individual level ${ }^{30}$, thus ruling out the possibility that temporal dynamic patterns can differ across teams.

In Chapter 4, we have discussed the logical connections between group-level and individual-level temporal dynamics of a psychological attribute within the conventional inter-subject approach that assumes homogeneous individual dynamic patterns, and a newly developed intra-subject longitudinal approach that assumes individual heterogeneity. We further elaborated the intra-subject approach with an example of attributes repeatedly measured three times. In Chapter 5 we applied both the inter-subject and intra-subject approaches in a longitudinal study of project teams and examined the impact of team conflict over time on team performance and team satisfaction, following the theoretical premises of the punctuated equilibrium model (Gersick, 1988, 1989, 1991). The outcomes of this study were notable. Whereas the inter-subject approach allowed us to replicate findings from a similar study by Jehn and Mannix (2001), the intra-subject approach revealed large differences in conflict dynamics across individual teams. Most strikingly, the dynamic pattern of task conflict, relationship conflict, and process conflict found at the group level applied only to $5 \%, 10 \%$ and $2 \%$ of the teams, respectively. This evidence clearly demonstrates the heterogeneity of team process dynamics over time and resonate Hackman's (1987) earlier argument concerning the idiosyncrasy of individual team processes. Comparing between the two approaches, we noted that intrateam temporal dynamics of conflict, as established with the intra-subject longitudinal approach, has a unique impact on team satisfaction. This effect is unrelated to the effect of team conflict level at different time moments, as assessed with the inter-subject longitudinal approach (used by Jehn and Mannix, 2001). We conclude that the intra-subject approach does not only conceptually and logically suit better the assessment of team process dynamics over time and its nomological networks with team inputs and team outputs, but also complements the conventional intersubject longitudinal approach.

\footnotetext{
${ }^{30}$ Group- and individual-level refers to a group (e.g. sample, subsample) of teams and individual teams.
} 


\section{Implications for Future Research}

The methods and findings presented in preceding chapters contribute to team research in several ways. First, we propose alternative tests and develop new analytical tools that can be added into team researcher's methodological toolkit and provide suggestions for model building, research design, and analysis that fit the temporal-forward logic of the IPO-framework-based team research. Second, we promote a more temporal way of thinking and suggest new and more rigorous ways to incorporate time in team research. We argue that such practice is in line with organizational theorists (McGrath \& Tschan, 2004; Roe, 2008; Van de Ven, 2007) recent calls for process-based temporal research. We elaborate on these contributions as below.

\section{Using Team Inputs as Predictors}

Previous IPO-framework-based team research has identified a wide range of individual characteristics that define team composition, or, in other words, team inputs. While early studies focused on "surface-level" demographic characteristics such as age, gender, ethnicity, and functional background, later studies added "deep-level" psychological characteristics, such as values, attitudes, personality traits, into the notion of team inputs. More recently, temporal characteristics have been added, including time urgency, polychronicity, time perspective, and temporal depth. Team inputs - demographic features or psychological attributes - have been conceptualized or examined as predictors of team outputs, having either a direct effect or an indirect effect intermediated by team processes. Some team inputs, especially, team psychological inputs, are often treated as mediators and moderators on the relationships between team demographic inputs and team processes or between team demographic inputs and team outputs.

Focusing on the temporal characteristics, we have argued for sufficient stability over time and across situations in order for team psychological inputs to make consistent predictions. However, such an argument pertains to all team characteristics. Even team demographic features should be scrutinized with regard to their stability. Stability is not an inherent quality of the construct corresponding to the attribute, but rather a quality of measurement, or, in other words, of measured attribute scores. Thus, the measurement instrument may not be equivalent. Moreover, the degree of stability is determined by the timeframe implied in pertinent theories and the temporal scope of the studies. An attribute like functional background or general hurry is more likely to be stable in a one-hour laboratory study than in a one-year field study. Furthermore, although not addressed in this dissertation, researchers should assess not only the equivalence (i.e. the occurrence of gamma and/or beta change) of measurement instruments, but also the stability (i.e. the occurrence of alpha change) in teams' multi-attribute profile, if team composition is 
defined by means of multiple attributes. It is likely that multi-attribute team composition is less stable than single-attribute team composition.

Another important issue is what low, or even a lack of, stability, as found in our studies, would imply. At the operational level, researchers should abstain from using single-moment measurements of such attributes and include team inputs demonstrating sufficient stability over time and across situations. But at the theoretical level, there is a more profound implication that is also more difficult to accommodate. If psychological attributes (or team demographic features) are unstable and open to change over time, they should be conceived and modeled as such, and their effects should be assessed in different ways, such as with the intra-subject approach. It is very likely that certain psychological attributes are dynamic and change under the influence of teams' learning or adaption processes or responses to internal or external events. We will refer to this issue when discussing about more rigorous ways of doing processes-based temporal research.

\section{Examining Heterogeneous Temporal Dynamic Patterns of a Team Process}

The IPO framework suggests the team processes, that is, team internal and external interactions over time (Marks et al, 2001), mediate and moderate the effect of team inputs - surface-level demographic features or deep-level psychological attributes - on team performance and social outputs. The concepts of mediation and moderation are part of the differential research paradigm, and the proposed mediation and moderation effects can only be established, if team processes are conceptualized and measured in a way that allows for inter-team differences in intra-team process dynamics over time. So far, research based on the IPO-framework has not achieved this, and team researchers have struggled with how to fit the notion of dynamic team processes in an otherwise differential design. Inter-team differences in intra-team process dynamics have been assessed with proxies (e.g. Harrison et al. 2002) and single moment measures (e.g. Jehn et al., 1999; Pelled et al., 1999), but such research practice does not grasp the dynamic nature of team processes. Descriptive studies and qualitative analyses (e.g. Tuckman, 1965; Gersick, 1988, 1991; Ericksen \& Dyer, 2004) do not examine such inter-team differences, neither does the inter-subject approach with temporally backward reasoning (e.g. Jehn \& Mannix, 2001). Proposing an intra-team longitudinal approach as an alternative to the inter-subject longitudinal approach, we have argued that team processes should be conceptualized and studied as a holistic phenomenon and concept that is temporally integrated and undividable by discrete time moments within each team. We further devised an analysis method that starts from identifying each team's dynamic pattern of a process over time, and applies hierarchical clustering analysis to identify clusters of teams based on their process dynamic patterns. With this method we obtain a categorical variable that captures different process dynamic patterns across teams and are able to enter the categorical variable into traditional multivariate analysis methods to examine its nomological networks with team input antecedents and team 
output consequences. Hence, the intra-subject approach provides a solution to a crucial problem that has been hitherto unresolved and has hindered the inclusion of team processes from improving team effectiveness models.

It is noteworthy that the results of the two approaches are rather different and point at unrelated inter-team differences. While the intra-subject approach shows the impact of team process dynamics over time on team outputs, the inter-subject approach shows the impact of team process level at respective time moments on team outputs. We have found that particular team conflict dynamic patterns are harmful to team satisfaction, regardless team conflict level, and that the "typical" conflict dynamic pattern of high- or low-satisfaction teams are an aggregated pattern consolidating various individual patterns. These reported differential effects resonate with Van de Ven's (2007) calls for studying organizational phenomena with both dynamic processes-based models and static variances-based models in order to obtain comprehensive knowledge of the researched phenomena. He argues that whereas variances-based models help to answer what factors affect a researched phenomenon and to what degree, processes-based models help to answer how antecedents, identified in variances-based models, exercise their influence. Our findings support his proposition and suggest that results from the intra-subject approach and those from the inter-subject approach complement each other in providing insights of a researched phenomenon. Not only should within-subject variability over time and between-subjects heterogeneity at respective time moments be distinguished at the conceptual level, but compatible research approaches should also be applied to respective theoretical inquires. This means using the intra-subject longitudinal approach to examine within-subject variability over time and using the intersubject longitudinal approach to examine between-subjects heterogeneity at respective time moments.

\section{Analyzing the Role of Team Processes}

A crucial question for future team research is how to examine the role of team processes that are conceived as teams' internal and external interactions over time and measured as dynamic patterns. With the developed intra-subject approach, pertinent analyses can be done in a rather straightforward manner. Assuming that team input variables (and team output variables!) are stable over time, team researchers can categorize teams on the basis of their dynamic patterns over time, and subsequently use multivariate analysis techniques to examine how team input levels relate to particular process dynamic patterns and how process dynamic patterns relate to team output levels. Drawing upon previous literature (e.g. De Wit \& Greer, 2008; De Dreu \& Weingart, 2003), we exemplify the intra-subject approach with a study in which team members' diversity in functional background and team tenure constitutes a team input, the temporal dynamics of task conflict is a team process, and team performance is a team output. To analyze the relationship between team inputs and team processes, researchers can use polynomial logistic regression, because the 
temporal dynamics of team task conflict is operationalized as a categorical variable. Researchers would estimate the model

$$
Y=\alpha+\beta_{1} \cdot X_{1}+\beta_{2} \cdot X_{2}+\beta_{3} \cdot X_{3}+\varepsilon
$$

where

$$
\begin{aligned}
& \mathrm{Y}=\text { Team task conflict dynamics } \\
& \mathrm{X}_{1}=\text { Control variable } \\
& \mathrm{X}_{2}=\text { Team functional background diversity } \\
& \mathrm{X}_{3}=\text { Team tenure diversity } \\
& \varepsilon=\text { Error term }
\end{aligned}
$$

To examine the impact of team task conflict dynamics over time on team performance, researchers would estimate the model underneath with Multivariate Variance of Analysis (MANOVA) or linear regression with dummy variables.

$$
\mathrm{Z}=\alpha+\beta_{1} \cdot \mathrm{V}+\beta_{2} . \mathrm{Y}+\varepsilon
$$

where

$$
\begin{aligned}
& Z=\text { Team performance } \\
& V=\text { Control variable } \\
& Y=\text { Team task conflict dynamics } \\
& \varepsilon=\text { Error term }
\end{aligned}
$$

The applicability of these analysis methods is limited by practical constraints. For example, if the sample size of a study is too small to estimate the above models with the 17-pattern categorization scheme for three-moment dynamics, researchers could use the three broader categorization schemes that are based on change direction, change degree, and change rate (p.79-82). Model estimation with the 17pattern scheme informs what impact one particular temporal dynamic pattern of team task conflict exerts on team performance. Model estimation with each of the three broader schemes informs what impact one particular feature (i.e. change direction, change degree, change rate) of team task conflict dynamics exerts on team performance.

Moreover, the models estimated with the intra-subject longitudinal approach differ from those estimated with the conventional inter-subject longitudinal approach. The difference is exemplified with a study in which team performance is predicted by team demographic diversity (e.g. team diversity in gender, age and 
ethnic) and team task conflict over time serves as a mediator ${ }^{31}$. With the intrasubject approach, researchers estimate the model

$$
\mathrm{Y}=\alpha+\beta_{1} * \mathrm{X}_{1}+\beta_{2} * \mathrm{X}_{2}+\beta_{3} * \mathrm{X}_{3}+\beta_{4} * \mathrm{X}_{4}+\beta_{5} * \mathrm{X}_{5}+\varepsilon
$$

where

$$
\begin{aligned}
& \mathrm{Y}=\text { Team performance } \\
& \mathrm{X}_{1}=\text { Control variable } \\
& \mathrm{X}_{2}=\text { Gender diversity } \\
& \mathrm{X}_{3}=\text { Age diversity } \\
& \mathrm{X}_{4}=\text { Ethnic diversity } \\
& \mathrm{X}_{5}=\text { Task conflict dynamics } \\
& \varepsilon=\text { Error term }
\end{aligned}
$$

In contrast, the model to be estimated in the inter-subject approach is

$$
\mathrm{Y}=\alpha+\beta_{1} * \mathrm{X}_{1}+\beta_{2} * \mathrm{X}_{2}+\beta_{3} * \mathrm{X}_{3}+\beta_{4} * \mathrm{X}_{4}+\beta_{5} * \mathrm{X}_{5}+\beta_{6} * \mathrm{X}_{6}+\beta_{7} * \mathrm{X}_{7}+\varepsilon
$$

where

$$
\begin{aligned}
& \mathrm{Y}=\text { Team performance } \\
& \mathrm{X}_{1}=\text { Control variable } \\
& \mathrm{X}_{2}=\text { Gender diversity } \\
& \mathrm{X}_{3}=\text { Age diversity } \\
& \mathrm{X}_{4}=\text { Ethnic diversity } \\
& \mathrm{X}_{5}=\text { Task conflict at } \mathrm{T} 1 \\
& \mathrm{X}_{6}=\text { Task conflict at } \mathrm{T} 2 \\
& \mathrm{X}_{7}=\text { Task conflict at } \mathrm{T} 3 \\
& \varepsilon=\text { Error term }
\end{aligned}
$$

Moreover, the intra-subject approach can also be used to explore the nomological networks of intra-team process dynamics over time and help to understand the interrelations among different team processes, for example, among team communication patterns, conflict patterns, and trust development patterns, and how team process dynamics relate to team performance and team social outputs. We discuss the intra-subject approach with more than one dynamic variable and more than three time moments elsewhere.

\footnotetext{
${ }^{31}$ In addition to this model, testing the mediation effect also involves testing the effect of independent variables on the mediator, the effect of the mediator on the dependent variable, and the effect of independent variable on the dependent variable.
} 


\section{Other Implications}

Our work has several other implications for team research. First, it suggests that the assumption of high stability of team attributes - although often made - is not always met in team research. Alerted by the findings of our empirical studies, researchers may argue that the assumed high stability is often plausible when the researched teams have rather short life spans. Many real-life teams often exist for months or years and may change in composition over time and/or change in their interaction patterns due to learning, adaptation, or exposure to internal and external team events. The same reasoning can also be applied to team outputs. In other words, team outputs, such as team performance, may also demonstrate low intertemporal stability and unfold differently over time across teams of long life spans.

Stability seems to be a boundary condition of the inter-subject longitudinal approach. If team inputs and team outputs are stable, researchers may continue to apply the inter-subject approach with the differential logic and examine the between-teams covariance among team input level, team process dynamics, and team output levels. However, if team inputs and/or team outputs are unstable over time, the inter-subject approach will not produce meaningful and consistent results and researchers may need an alternative approach. It is beyond the scope of this dissertation to elaborate on the alternative approach, but we can refer to the publications by Van de Ven (2007) about "process research" and by Roe $(2008,2009)$ and Solinger (2010) about the "temporalist approach". From a radical temporalist perspective, all work-related and organizational phenomena should be conceived as dynamic and studied with compatible methods. In the context of the-IPO-frameworkbased team research, researchers need to first depict the temporal dynamic patterns for team inputs, team processes, and team outputs, and then examine their interrelations with longer and shorter time lags, using appropriate time frames and grids.

The implications of this dissertation are not limited to team research but indicate the necessity to examine intertemporal stability and measurement equivalence before assessing stability in other research domains of organizational behaviors. The analysis methods that we have described can be applied to study, for example, the temporal dynamics of competence or commitment. Likewise, the intra-subject longitudinal approach can be applied to study intra-subject processes over time in contrast to inter-subject variation of individual attributes therein. It is also noteworthy that that latent growth modeling, although gaining popularity in organizational behavior research (Chan, 1998), assumes that individual growth trajectories are random variations of the group trajectory, an assumption may not hold or be fruitful in studying intra-subject attribute change over time. Although in some cases, latent growth modeling and its embedded group-to-individuals logic may make sense, researchers should also be aware alternative analysis methods that are based on the individuals-to-group logic and the insights these methods can produce. 


\section{Managerial Implications}

We derive two major managerial implications from the research presented in this dissertation. First, the low intertemporal and intersituational stability reported in the studies suggests that organizations may have an impact on how teams are composed and function. It may even indicate how much effort organizations need to make in order to change organization participants' time-related attitudes, behaviors, and traits to the desirable kind and level and cultivate desirable time-related norms and cultures within organizations. Results from the dissertation suggest that it is rather difficult to change individuals' future orientation and cognitive lengths into the past and future but relatively easy to pressure individuals with deadlines, encourage their competitiveness, adapt their working speed, cultivate their multitasking preference, and change their positive and negative views of the past, and hedonistic views of the present. By using multiple deadlines strategically and providing proper training programs of time management, organizations are able to improve employees' work efficiency and consequently improve their work performance and psychological well-beings (Hecht \& Allen, 2005; Madjar \& Oldham, 2006).

Second, regarding project team management, it is important to monitor and manage the level and dynamics of team task, relationship, and process conflict over team projects If team satisfaction is the major concern, it is crucial to lower team task and process conflict from the middle time point to the end of team projects and relationship conflict level throughout team projects via various conflict resolution tactics, such as focusing on the content of interpersonal interactions and assigning individual tasks according to expertise (Behfar et al., 2008).

\section{Limitations}

This dissertation bears three major limitations. The first one pertains to the generalizability of the reported empirical findings (i.e. findings in Chapter 2, 3, 5) to other sample types than academia and university students and to other time intervals than those in the dissertation. The rhythm of Maastricht University's academic calendar regulate the activities of most of our respondents; hence, replication of the studies are necessary for better understanding of the intertemporal and intersituational stability of time urgency, polychronicity, time perspective, and temporal depth. The sample of student project team limits the generalizability of reported findings on team conflict over time to other types of project teams, despite the "versatility" of punctuated equilibrium theory (Van de Ven, 2007). Likewise, the generalizability of our findings is limited to six months for the intertemporal stability of the four temporal characteristics, one month for the intersituational stability of the four temporal characteristics, and two months for team conflict over time. Again, 
replication of the studies over different time intervals with academia and university student samples is necessary for cross-validating the reported findings.

Second, small sample sizes in the studies have challenged the validity of the reported findings. Longitudinal design with repeated measurement often subject to small sample sizes due to high dropout rates over time. For the intertemporal stability study (Chapter 2), 50\% of the initial respondents dropped out from the study in the end; for the intersituational stability study (Chapter 3), high dropout rate even forced us to change our original analysis strategy from using the three-moment data to using the first-two-moment data instead. Participant dropout presents a particular threat to longitudinal research. The threat of high participant dropout rate is particularly harmful to quantitative team research with repeated measurement and primary data source. Such research requires sufficiently large sample sizes and complete teams' data, but often subjects to high participant dropout and measurement errors of the included variables. To overcome this threat, researchers may draw the initial samples from large sample pools, use attractive incentive schemes to encourage continuous participation, pilot-test, and engage research stakeholders (e.g. users of final research results) in designing research. On the other hand, it is also important for the team research community to recognize the difficulty of this particular research stream and its potential contribution in advancing extant team theories.

Finally, the intra-subject longitudinal approach (Chapter 4, 5) applies only to team process dynamics defined by three time moments. Dynamics defined by more than three moments need more than 17 possible patterns to describe. In other words, this approach is most applicable to team research that draws upon punctuated equilibrium theory and presumes the beginning, middle and end of team projects are three most critical time moments of team functioning. We discuss elsewhere the application of the intra-subject approach to longitudinal team research in which team processes are repeatedly measured at more than three measurement moments and in which team inputs, team processes, and team outputs are all repeatedly measured three times. 


\section{References}

Ancona, D.G., \& Caldwell. D.F. 1992. Demography and design: Predictors of new product team performance. Organization Science, 3(3): 321-341.

Ancona, D.G., Okhuysen, G.A., \& Perlow, L.A. 2001. Taking time to integrate temporal research. Academy of Management Review, 26(4): 512-529.

Argyris, C. 1969. The incompleteness of social psychological theory: Examples from small group, cognitive consistency, and attribution research. American Psychologist, 24: 893-908.

Bales, R.F. 1950. Interaction process analysis: A method for the study of small groups. Cambridge, Mass.: Addison-Wesley.

Barsade, S.G., Ward, A.J., Turner, J.D.R., \& Sonnenfeld, J.A. 2000. To your heart's content: A model of affective diversity in top management teams. Administrative Science Quarterly, 45(4): 802-836.

Behfar, K.J., Peterson, R.S., Mannix, E.A., \& Trochim, W.M.K. 2008. The critical role of conflict resolution in teams: A close look at the links between conflict type, conflict management strategies, and team outcomes. Journal of Applied Psychology, 93(2): 170-188.

Bell, S.T. 2007. Deep-Level composition variables as predictors of team performance: A meta-analysis. Journal of Applied Psychology, 92(3): 595-615.

Bluedorn, A.C., 2002. The human organization of time: Temporal realities and experience, Stanford: Stanford University Press.

Bluedorn, A.C., Kalliah, T.J., Strube, M.J., \& Martin, G.D. 1998. Polychronicity and the inventory polychronic values (IPV). Journal of Managerial Psychology, 14 (3/4): 205-230.

Bluedorn, A.C., Kaufman, C.F., \& Lane, P.M. 1992. How many things do you like to do at once? An introduction to monochromic and polychromic time. Academy of Management Executive, 6: 17-26.

Bollen, K.A., 1989. Structural equations with latent variables, York: Wiley.

Boulding, K. 1963. Conflict and defense. New York: Harper \& Row.

Brown, S.L. \& Eisenhardt, K.M. 1995. Product development: Past research, present findings, and future directions. Academy of Management Review, 20(2): 343378.Byrne, D.E. 1971. The attraction paradigm. Orlando, FL: Academic Press.

Chan, D. 1998. The conceptualization and analysis of change over time: An integrative approach incorporating longitudinal mean and covariance structures analysis (LMACS) and multiple indicator latent growth modeling (MLGM). Organizational Research Methods, 1: 421-485. 
Chang, A., Bordia, P., \& Duck, J. 2003. Punctuated equilibrium and linear progression: Toward a new understanding of group development. Academy of Management Journal, 46(1): 106-117.

Cohen, S.G. \& Bailey, D.E. 1997. What makes teams work: Group effectiveness research from the shop floor to the executive suite. Journal of Management, 23(3): 239-290.

Conte, J.M., \& Gintoft, J.N. 2005. Polychronicity, Big Five Personality dimensions, and the sales performance. Human Performance, 18(4): 427-444.

Conte, J.M., \& Jacobs, R.R. 2003. Validity evidence linking polychronicity and Big Five Personality dimensions to absence, lateness, and supervisory performance rating. Human Performance, 16(2): 107-129.

Conte, J.M., Landy, F.J-., Mathieu, J.E.1995. Time urgency: Conceptual and construct development. Journal of Applied Psychology, 80(1): 178-185.

Conte, J.M., Mathieu, J.E., \& Landy, F.J. 1998. The nomological and predictive validity of time urgency. Journal of Organizational Behavior, 19(1):1-

Conte, J.M., Ringenbach, K.L., Moran, S.K. \& Landy, F.J. 2001. Criterion-validity evidence for time urgency: Associations with burnout, organizational commitment, and job involvement in travel agents. Applied H.R.M. Research, 6(12): 129-134.

Costa, P.T., Jr, \& McCrae, R.R. 1988, P.T., Jr., \& McCrae, R.R. Personality in adulthood: A six-year longitudinal study of self-reports and spouse ratings on the NEO personality inventory. Journal of Personality and Social Psychology, 54, 853-863.

Costa, P.T., Jr., \& McCrae, R.R. 1992. Set like plaster? Evidence for the stability of adult personality. In T.F. Heatherton, \& J.L. Weinberger (Eds.) Can personality change? Washington D.C.: American Psychological Association.

Dalal, R.S., Lam, H., Weiss, H.M., Welch, E.R., \&Hulin, C.L. 2009. A withinperson approach to work behavior and performance: Concurrent and lagged citizenship-counterproductivity associations, and dynamic relationships with affect and overall job performance. Academy of Management Journal, 52(5): 1051-1066.

Das, T.K. 1987. Strategic planning and individual temporal orientation. Strategic Management Journal, 8(2): 203-209.

Davis-Blake, A., \& Pfeffer, J. 1989. Just a mirage: The search for trait effects in organizational research. Academy of Management Review, 14(3): 385-400.

De Dreu, C.K. W., \& Weingart, L.R. 2003. Task versus relationship conflict, team performance, and team member satisfaction: A meta-analysis. Journal of Applied Psychology, 88(4): 741-749.

De Wit, F.R.C., \& Greer, L.L., 2008. The black-box deciphered: A meta-analysis of team diversity, conflict, and team performance. The Annual Meeting of Academy of Management: Anaheim, C.A.

Dishon-Berkovits, M., \& Koslowsky, M. 2002. Determinants of employee punctuality. Journal of Social Psychology, 142(6): 723-739.

Donaldson, T. 1996. Values in tension: Ethics away from home. Harvard Business Review, 74(5): 48-62. 
Eccles, R.G., Newquist, S.C., \& Schatz, R. 2007. Reputation and its risks. Harvard Business Review, 85(2): 104-114.

Eisenhardt, K. M., \& Bourgeois III, L.J. 1988. Politics of strategic decision making in high-velocity environments: Toward a midrange theory. Academy of Management Journal, 31(4): 737-770.

El Sawy, O.A. 1983. Temporal perspective and managerial attention: A study of chief executive strategic behavior, Dissertation Abstracts International 44 (05A): 1556-1557.

Epel, E.S., Bandura, A., \& Zimbardo, P.G. 1999. Escaping homelessness: The influence of self-efficacy and time perspective on coping with homelessness. Journal of Applied Social Psychology, 29(3): 575-596.

Ericksen, J., \& Dyer, L. 2004. Right from the start: Exploring the effects of early team events on subsequent project team development and performance. Administrative Science Quarterly, 49: 438-471.

Ford, C., \& Sullivan, D.M. 2004. A time for everything: how the timing of novel contributions influences project team outcomes. Journal of Organizational Behavior, 25(2): 279-292.

Friedman, M., \& Rosenman, R. 1974. Type A behavior and your heart. New York: Knof.

George, J.M., \& Jones, G.R. 2000. The role of time in theory and theory building. Journal of Management, 26(4): 657-684.

Gersick, C.J.G. 1988. Time and transition in work teams: Toward a new model of group development. Academy of Management Journal, 31(1): 9-41.

Gersick, C.J.G. 1989. Marking time: Predicable transitions in task groups. Academy of Management Journal, 32(2): 274-309.

Gersick, C.J.G. 1991. Revolutionary change theories: A multilevel exploration of the punctuated equilibrium paradigm. Academy of Management Review, 16(1): 10-36.

Golembiewski, R.T., Billingsley, K., \& Yeager, S. 1976. Measuring change and persistence in human affairs: Types of change generated by OD designs. Journal of Applied Behavioral Science, 12: 133-157.

Greenberg, J. 2002. Time urgency and job performance: Field evidence of an interactionist perspective. Journal of Applied Social Psychology, 32(9): 1964-1973.

Greer, L.L., Jehn, K.A., \& Mannix, E.A. 2008. Conflict transformation: A longitudinal investigation of the relationships between different types of intragroup conflict and the moderating role of conflict resolution. Small Group Research, 39(3): 278-302.

Hackman, J.R. 1987. The design of work teams. In J. W. Lorsch (Eds.), Handbook of Organizational Behavior. 315-342: Englewood Cliffs, NJ: Prentice-Hall.

Hall, E.T., 1983. The dance of life, New York: Doubleday.

Hambrick, D.C., \& Mason, P.A. 1984. Upper echelons: The organization as a reflection of its top managers. Academy of Management Review, 9(2): 193-206.

Harber, K.D., Zimbardo, P.G., Boyd, J.N. 2003. Participant self-selection biases as a function of Individual differences in time perspective. Basic and Applied Social Psychology, 25(3): 255-264. 
Harrison, D.A., Price, K.H., \& Bell, M.P. 1998. Beyond relational demography: Time and the effects of surface-level and deep-level diversity on work group cohesion. Academy of Management Journal, 41(1): 96-107.

Harrison, D.A., Price, K.H., Gavin J.H., \& Florey, A.T. 2002. Time, teams, and task performance: Changing effects of surface-level and deep-level diversity on group functioning. Academy of Management Journal, 45(5): 1029-1045.

Hendriks, A.A.J., Hofstee, W.K.B., \& De Raad, B. 1999. The Five-Factor Personality Inventory (FFPI). Personality and Individual Differences, 27: 307-325.

Hecht, T.D., \& Allen, N.J. 2005. Exploring links between polychronicity and wellbeing from the perspective of person-job fit: Does it matter if you prefer to do only one thing at a time? Organizational Behavior and Human Decision Processes, 98(2): 155-178.

House, R.J., Shane, S.A., \& Herold, D.M. 1996. Rumors of the death of trait research are vastly exaggerated. Academy of Management Review, 21(1): 203224.

Hulsheger, U.R., Anderson, N., \& Salgado, J.F. 2009. Team-level predictors of innovation at work: A comprehensive meta-analysis spanning three decades of research. Journal of Applied Psychology, 94(5): 1128-1145.

Ilgen, D.R., Hollenbeck, J.R., Johnson, M., \& Jundt, D. 2005. Teams in organizations: From Input-Process-Output models to IMOI models. Annual Review of Psychology, 56(1): 517-543.

Janis, I.L. 1972. Victims of groupthink: A psychological study of foreign-policy decisions and fiascoes. Boston: Houghton Mifflin.

Jehn, K.A. 1994. Enhancing effectiveness: An investigation of advantages and disadvantages of value-based intragroup conflict. International Journal of Conflict Management, 5, 223-238.

Jehn, K.A. 1995. A multimethod examination of the benefits and detriments of intragroup conflict. Administrative Science Quarterly, 40(2): 256-282.

Jehn, K.A. 1997. A qualitative analysis of conflict types and dimensions in organizational groups. Administrative Science Quarterly, 42(3): 530-557.

Jehn, K.A., \& Mannix, E.A. 2001. The dynamic nature of conflict: A longitudinal study of intragroup conflict and group performance. Academy of Management Journal, 44(2): 238-251.

Jehn, K.A., Northcraft, G.B., \& Neale, M.A. 1999. Why differences make a difference: A field study of diversity, conflict, and performance in workgroups. Administrative Science Quarterly, 44(4): 741-763.

Jenkins, C.D., Zyzanski, S.J., \& Rosenman, R.H. 1971. Progress toward validation of a computer-scored test for the Type A coronary-prone behavior pattern. Psychosomatic Medicine, 33: 193-202.

Jourdan, F. 1993. Test-retest measurement of the Zimbardo Time Perspective Inventory. Unpublished raw data.

Kellerman, B. 2006. When should a leader apologize and when not? Harvard Business Review, 84(4): 72-81.

Keough, K.A. 1993. Emotions and moods as filtered through the lens of time perspective and gender. Unpublished manuscript, Stanford University. 
Keough, K.A., Zimbardo, P.G., \& Boyd, J.N. 1999. Who’s smoking, drinking, and using drugs? Time perspective as a predictor of substance use. Basic and Applied Social Psychology. 21(2): 149-164.

Konig, C.J., Buhner, M., \& Murling, G. 2005. Working memory, fluid intelligence, and attention are predictors of multitasking performance, but polychronicity and extraversion are not. Human Performance, 18(3): 243-266.

Kozlowski, S. W. J. \& Ilgen, D.R. 2006). Enhancing the effectiveness of work groups and teams. Psychological Science in the Public Interest, 7(3): 77-124.

Lance, C.E., Vandenberg, R.J., Self, R.M. 2000. Latent growth models of individual change: The case of newcomer adjustment. Organizational Behavior and Human Decision Processes, 83(1): 107-140.

Landy, F.J., Rastegary, H., Thayer, J., \& Colvin, C. 1991. Time urgency: The construct and its measurement. Journal of Applied Psychology, 76(5): 644- 657.

Lawrence, B.S. 1997. The black box of organizational demography. Organization Science, 8(1): 1-22.

Lennings, C.J. 1991. The schalling sensation seeking and impulsivity scales: Their relationship to time perspective and time awareness: A preliminary report. Psychological Reports, 69: 131-136.

Lewin, K. 1951. Field theory in the social sciences: Selected theoretical papers. New York: Harper.

Madjar, N., \& Oldham, G.R. 2006. Task rotation and polychronicity: effects on individuals' creativity. Human Performance, 19(2): 117-131.

Marks, M.A., Mathieu, J.E., \& Zaccaro, S.J. 2001. A temporally based framework and taxonomy of team processes. Academy of Management Review, 26(3): 356-376.

McGrath, J.E. 1984. Groups: Interaction and performance. Englewood Cliffs, NJ: Prentice-Hall.

McGrath, J.E. 1986. The place of time in social psychology: New perspectives. In J.E. McGrath (Eds.) The social psychology of time: New perspectives. Newbury Park, CA: Sage.

McGrath, J.E., \& Tschan, F. 2004. Temporal matters in social psychology: Examining the role of time in the lives of groups and individuals. Washington D.C.: American Psychological Association.

Mitchell, T.R., \& James, L.R. 2001. Building better theory: Time and the specification of when things happen. Academy of Management Review, 26(4): 530-547.

Mohammed, S., \& Angell, L.C. 2004. Surface- and deep-level diversity in workgroups: Examining the moderating effects of team orientation and team process on relationship conflict. Journal of Organizational Behavior, 25(8): 10151039.

Onken, M.H. 1999. Temporal elements of organizational culture and impact on firm performance. Journal of Managerial Psychology, 14(3/4): 231-244.

O'Reilly Ill, C.A., Caldwell, D.F., \& Barnett, W.P.1989. Work group demography, social integration, and turnover. Administrative Science Quarterly, 34(1): 2137.

Pearson, C.M., \& Clair J.A. 1998. Reframing crisis management. Academy of Management Review, 23(1): 59-76. 
Pelled, L.H. 1996. Demographic diversity, conflict, and work group outcomes: An intervening process theory. Organization Science, 7(6): 615-631.

Pelled, L. H., Eisenhardt, K. M., \& Xin, K. R. 1999. Exploring the black box: An analysis of work group diversity, conflict, and performance. Administrative Science Quarterly, 44(1): 1-28.

Poole, M., \& Cooney, G. 1987. Orientation to the future: A comparison of adolescents in Australia and Singapore, Journal of Youth and Adolescence, 16: 129151.

Raes, A.M.L.2009, Top management team impact on organizations: Determinants from inside and outside the boardroom. Doctoral dissertation, Maastricht University, The Netherlands.

Roe, R.A. 2008. Time in applied psychology: The study of "what happens" rather than what "what is." European Psychologist, 13(1): 37-52.

Roe, R. 2009. Perspectives on time and the chronometric study of what happens in organizations. In R.A. Roe, M.J. Waller \& S.R. Clegg (Eds.) Time is organizational research. Oxfordshire, U.K.: Routledge.

Rudelius, W., \& Buchholz, R.A 1979. Ethical problems of purchasing managers. Harvard Business Review, 57(2): 8-14.

Seers, A., \& Woodruff, S. 1997. Temporal pacing in task forces: Group development or deadline pressure? Journal of Management, 23(2): 169-187.

Singer, J.D. \& Willet, J.B. 2003. Applied longitudinal data analysis: Modeling change and event occurrence. Oxford: Oxford University Press.

Slocombe, T.E., \& Bluedorn, A.C. 1999. Organizational behavior implications of the congruence between preferred polychronicity and experienced work-unit polychronicity. Journal of Organizational Behavior, 20(1): 75.

Smith, N.C., Thomas, R.J., \& Quelch, J.A. 1996. A Strategic approach to managing product recalls. Harvard Business Review, 74(5): 102-112.

Solinger, O.N. 2010. Capturing the dynamics of commitment: Temporal theory, method, and an application to the organizational commitment attitude. Doctoral dissertation, Maastricht University, The Netherlands.

Tajfel, H. 1982. Social psychology of intergroup relations. Annual Review of Psychology, 33: 1-39.

Taylor, M.S., Locke, E.A., Lee, C., \& Gist, M.E. 1984. Type A behavior and faculty research productivity: What are the mechanisms? Organizational Behavior and Human Performance, 34(3): 402-418.

Tsui, A.S., Egan, T.D., \& O’Reilly, C.A. 1992. Being different: Relational demography and organizational attachment. Administrative Science Quarterly, 37(1): 549-579.

Tuckman, B.W. 1965. Developmental sequences in small groups. Psychological Bulletin, 63: 384-399.

Van de Ven, A.H. 2007. Engaged scholarship: A guide for organizational and social research. New York: Oxford University Press Inc.

Wagner, W.G., Pfeffer, J., \& O’Reilly III, C.A. 1984. Organizational demography and turnover in top-management groups. Administrative Science Quarterly, 29(1): 74-92. 
Waller, M.J., Conte, J.M., Gibson, C.B., \& Carpenter, M.A. 2001. The effect of individual perceptions of deadlines on team performance. Academy o Management Review, 26(4): 586-600.

Waller, M.J. Giambatista, R.C., \& Zellmer-Bruhn, M.E. 1999. The effects of individual time urgency on group polychronicity. Journal of Managerial Psychology, 14(3/4): 244-257.

Waller, M.J., Zellmer-Bruhn, M.E., \& Giambatista, R.C. 2002. Watching the clock: Group pacing behavior under dynamic deadline. Academy of Management Journal, 45(5): 1046-1055.

Wheelan, S.A. 1994. Group processes: A developmental perspective. Sydney: Allyn \& Bacon.

Wiersema, M.F., \& Bantel, K.A. 1992. Top management team demography and corporate strategic change. Academy of Management Journal, 35(1): 91-121.

Wills, T.A., Sandy, J.M., \& Yaeger, A.M. 2001. Time perspective and early- onset substance use: A model based on stress-coping theory. Psychology of Addictive Behaviors, 15(2): 118-125.

Zaheer, S., Albert, S., \& Zaheer, A. 1999. Time scales and organizational theory. Academy of Management Review, 24(4): 725-741.

Zimbardo, P.G., \& Boyd, J.N. 1999. Putting time in perspective: A valid, reliable individual differences metric. Journal of Personality and Social Psychology, 77(6): 1271-1288. 



\section{Appendix A The Measurement of the Included Constructs}

\section{The Time Urgency Scale}

Please indicate to what extent you agree with the following descriptions of yourself. Strongly Disagree, Disagree, Neutral, Agree, Strongly Agree

\section{The Eating Behavior Items}

I eat too quickly.

I eat rapidly, even when there is plenty of time.

My spouse or a friend told me that I eat too fast.

I eat more slowly than most people. $(\mathrm{R})^{32}$

I prefer to linger over a meal and enjoy it. (R)

\section{The Competitiveness Items}

I am ambitious.

I am hard driving.

I am hard driving and competitive.

I have a strong need to excel in most things.

I go "all out".

I am bossy and dominating. ${ }^{33}$

\section{The General Hurry Items}

I am usually pressed for time.

I often feel very pressed for time.

I never feel in a rush, even under pressure. (R)

\section{The Task-Related Hurry Items}

I usually work fast.

I ordinarily work quickly and energetically.

I am slow doing things. (R)

\section{The Polychronicity Scale}

Please indicate to what extent you agree with the following descriptions of yourself. Strongly Disagree, Disagree, Neutral, Agree, Strongly Agree

I am comfortable doing several things at the same time.

When I sit down at my desk, I work on one project at a time. (R)

I do not like juggle several activities at the same time. (R)

People should not try to do many things at once. (R)

\footnotetext{
${ }^{32}$ In the Appendix section, the letter $\mathrm{R}$ refers to reversed wording, which is not shown the in the questionnaire presented to the participants of the studies.

${ }^{33}$ The item was excluded from the stability test.
} 


\section{The Time Perspective Scale}

Please indicate to what extent you agree with the following descriptions of yourself. Strongly Disagree, Disagree, Neutral, Agree, Strongly Agree

\section{The Past-Positive Items}

It gives me pleasure to think about my past.

I enjoy stories about how things use to be in the "good old times".

I get nostalgic about my childhood.

Happy memories of good times spring readily to mind.

Familiar childhood sights, sounds, smells often bring back a flood of wonderful memories.

On balance, there is much more good to recall than bad in my past. ${ }^{34}$

\section{The Past-Negative Items}

I think about the bad things that have happened to me in the past.

I think about the good things that I have missed out in my life.

I often think of what I should have done differently in my life.

It is hard for me to forget unpleasant images of my youth.

Painful past experiences keep being replayed in my mind.

\section{The Present-Hedonistic Items}

I take risks to put excitement in my life.

Taking risks keeps my life from becoming boring.

It is important to put excitement in my life.

\section{The Present-Fatalistic Items}

You can't really plan for the future because things change so much.

It doesn't make sense to worry about the future, since there is nothing that I can do about it anyway.

My life path is controlled by forces I cannot influence.

Since whatever will be will be, it doesn't really matter what I do.

\section{The Future-Oriented Items}

I am able to resist temptations when I know that there is work to be done.

When I want to achieve something, I set goals and consider specific means for reaching those goals.

I complete projects on time by making steady progress.

Meeting tomorrow's deadline and doing other necessary work comes before tonight's play.

\footnotetext{
${ }^{34}$ The item was excluded in the stability test.
} 


\section{The Temporal Depth Scale}

Please choose a time period from the options below to replace "(this)" in each of the following sentences.

One day, one week, two weeks, one month, three months, six months, nine months, one year, three years, five years, ten years, fifteen years, twenty years, twenty-five years, and more than twenty-five years.

When I think about the short-term future, I usually think about things (this) ahead. When I think about the mid-term future, I usually think about things (this) ahead. When I think about the long-term future, I usually think about things (this) ahead. When I think about things that happened recently, I usually think about things that happened (this) ago.

When I think about things that happened a middling time ago, I usually think about things that happened (this) ago.

When I think about things that happened a long time ago, I usually think about things that happened (this) ago.

\section{The Polychronicity Scale in the Computer Simulation}

Please indicate to what extent you agree with the following descriptions of yourself. Strongly Disagree, Disagree, Neutral, Agree, Strongly Agree

I am comfortable doing several things at the same time.

I believe it is best for people to be given several tasks and assignments to perform.

I believe it is best to complete one task before beginning another. (R)

When I sit down at my desk, I work on one project at a time. (R)

I do not like to juggle several activities at the same time. (R)

People should not try to do many things at once. (R)

\section{The Situational Strength Scale in the Computer Simulation}

Please indicate to what degree you would agree with the following statements.

Strongly Disagree, Disagree, Neutral, Agree, Strongly Agree

I felt anxious and nervous during the simulation.

I wanted to achieve the best performance during the simulation.

I wanted to finish the simulation as quickly as possible.

I was a bit worried about what results I would get from the simulation. 


\section{The Team Conflict Scale}

Please evaluate the functioning of your research team at this moment ${ }^{35}$ based on the following statements.

None, A Little, Some/Sometimes, Much, A Lot

\section{The Task Conflict Items}

How much conflict of ideas is there in your research team?

How often do people in your research team have conflicting opinions about the project your are working on?

How frequently do you have disagreements within your research team about the team assignment you are working on?

\section{The Relationship Conflict Items}

How much relationship tension is there in your research team?

How much emotional conflict is there in your research team?

How often do people get angry while working in your team?

\section{The Process Conflict Items}

How much conflict is there in your team about task responsibilities?

How often are there disagreements about who should do what in your research team?

How often do you disagree about resource allocation in your research team?

\section{The Team Satisfaction Scale}

Please indicate to what degree you would agree with the following statements.

Strongly Disagree, Disagree, Neutral, Agree, Strongly Agree

I am satisfied with working in my research team.

I would like to work with my team members on other team assignments in the future if given the opportunities to do so.

Overall our research team is doing a good job.

I really like my team members.

My team members and I have become friends after working together on the $\mathrm{IBR}^{36}$ team assignment.

\section{The Criteria for Grading Team Research Proposals}

\section{Content}

Introduction arouses interest in the research proposal.

Problem statement is not unfeasibly broad or too narrow to be of interest.

\footnotetext{
${ }^{35}$ The phrase "at this moment" is highlighted in the questionnaires.

${ }^{36}$ International Business Research is the name of the course, in which the data was gathered.
} 
Theoretical relevance: Problem analysis, inclusive links to other studies and theoretical considerations.

Practical relevance: Motivate research question in the light of the management dilemma.

Explanation and motivation of the research methodology in terms of the link to problem statement and feasibility.

\section{Format}

Meets academic writing standards, including syntax, punctuation, referencing, etc. Careful presentation. No sloppiness, errors.

Meets stated format requirements in the course book. 


\section{Appendix B}

\section{LISREL Syntax Chapter 2}

\section{The Estimation of M1}

\section{!ET M1}

Observed Variables

ET1_1 ET2_1 ET3_1 RET4_1 RET5_1 ET1_2 ET2_2 ET3_2 RET4_2 RET5_2 ET1_3 ET2_3 ET3_3 RET4_3 RET5_3

Raw Data from File ET.psf

Sample Size 63

Latent Variables

ET_1ET_2 ET_3

Equations

$$
\begin{aligned}
& \text { ET1_1=CONST+1 } * \text { ET_1 } \\
& \text { ET2_1=CONST+ET_1 } \\
& \text { ET3_1=CONST+ET_1 } \\
& \text { RET4_1=CONST+ET_1 } \\
& \text { RET5_1=CONST+ET_1 } \\
& \text { ET1_2=CONST+1 } * \text { ET_2 } \\
& \text { ET2_2=CONST+ET_2 } \\
& \text { ET3_2=CONST+ET_2 } \\
& \text { RET4_2=CONST+ET_2 } \\
& \text { RET5_2=CONST+ET_2 } \\
& \text { ET1_3=CONST+1 } * E T 33 \\
& \text { ET2_3=CONST+ET_3 } \\
& \text { ET3_3=CONST+ET_3 } \\
& \text { RET4_3=CONST+ET_3 } \\
& \text { RET5_3=CONST+ET_3 }
\end{aligned}
$$

Let the Errors between ET1_1 and ET1_2 Correlate Let the Errors between ET1_2 and ET1_3 Correlate Let the Errors between ET1_1 and ET1_3 Correlate Let the Errors between ET2_1 and ET2_2 Correlate Let the Errors between ET2_2 and ET2_3 Correlate Let the Errors between ET2_1 and ET2_3 Correlate Let the Errors between ET3_1 and ET3_2 Correlate Let the Errors between ET3_2 and ET3_3 Correlate 
Let the Errors between ET3_1 and ET3 3 Correlate

Let the Errors between RET4_1 and RET4_2 Correlate Let the Errors between RET4_2 and RET4_3 Correlate

Let the Errors between RET4_1 and RET4_3 Correlate Let the Errors between RET5_1 and RET5_2 Correlate

Let the Errors between RET5_2 and RET5_3 Correlate Let the Errors between RET5_1 and RET5_3 Correlate

Set the Error Variance of ET1_1 Free

Set the Error Variance of ET2_1 Free

Set the Error Variance of ET3_ 1 Free

Set the Error Variance of RET4_1 Free

Set the Error Variance of RET5_1 Free

Set the Error Variance of ET1_2 Free

Set the Error Variance of ET2_2 Free

Set the Error Variance of ET3_ 2 Free

Set the Error Variance of RET4_2 Free

Set the Error Variance of RET5_2 Free

Set the Error Variance of ET1_3 Free

Set the Error Variance of ET2_ 3 Free

Set the Error Variance of ET3_ 3 Free

Set the Error Variance of RET4_3 Free

Set the Error Variance of RET5_3 Free

Set the Variance of ET_1 Free

Set the Variance of ET_2 Free

Set the Variance of ET_3 Free

Set the Covariance between ET_1 and ET_2 Free

Set the Covariance between ET_2 and ET_3 Free

Set the Covariance between ET_1 and ET_3 Free

Path Diagram

End of Problem

\section{The Estimation of M2}

!ET M2

Observed Variables

ET1_1 ET2_1 ET3_1 RET4_1 RET5_1 ET1_2 ET2_2 ET3_2 RET4_2 RET5_2 ET1_3 ET2_3 ET3_3 RET4_3 RET5_3

Raw Data from File ET.psf 
Sample Size 63

Latent Variables

ET_1 ET_2ET_3

Equations

ET1_1 $=$ CONST $+1 * E T \_1$

ET2_1 $1=$ CONST $+0.97 *$ ET_ 1

ET3_1 $=$ CONST $+0.97 *$ ET_1

RET4_1 $=$ CONST $+0.67 *$ ET_1

RET5_1 $=$ CONST $+0.58 *$ ET_1

ET1_2=CONST $+1 * E T \_2$

$\mathrm{ET} 2 \_2=\mathrm{CONST}+0.97 * \mathrm{ET} \_2$

ET3_2 $=$ CONST $+0.97 *$ ET_2

RET4_2 $=$ CONST $+0.67 *$ ET_2

RET5_2 $=$ CONST $+0.58 *$ ET_2

ET1_3 $=$ CONST $+1 * E T \_3$

ET2_3 $=$ CONST $+0.97 *$ ET_3

ET3_3 $=$ CONST $+0.97 *$ ET_3

RET4_3 $=$ CONST $+0.67 *$ ET_3

RET5_3 $=$ CONST $+0.58 *$ ET_3

Let the Errors between ET1_1 and ET1_2 Correlate

Let the Errors between ET1_2 and ET1_3 Correlate Let the Errors between ET1_1 and ET1_3 Correlate

Let the Errors between ET2_1 and ET2_2 Correlate Let the Errors between ET2_2 and ET2_3 Correlate Let the Errors between ET2_1 and ET2_3 Correlate Let the Errors between ET3_1 and ET3_2 Correlate Let the Errors between ET3_2 and ET3_3 Correlate Let the Errors between ET3_1 and ET3_3 Correlate Let the Errors between RET4_1 and RET4_2 Correlate Let the Errors between RET4_2 and RET4_3 Correlate Let the Errors between RET4_1 and RET4_3 Correlate Let the Errors between RET5_1 and RET5 2 Correlate Let the Errors between RET5_2 and RET5_3 Correlate Let the Errors between RET5_1 and RET5_3 Correlate

Set the Error Variance of ET1_1 Free

Set the Error Variance of ET2_1 Free 
Set the Error Variance of ET3 1 Free

Set the Error Variance of RET4_1 Free

Set the Error Variance of RET5_1 Free

Set the Error Variance of ET1_2 Free

Set the Error Variance of ET2_2 Free

Set the Error Variance of ET3_ 2 Free

Set the Error Variance of RET4_2 Free

Set the Error Variance of RET5_2 Free

Set the Error Variance of ET1_3 Free

Set the Error Variance of ET2_3 Free

Set the Error Variance of ET3_ 3 Free

Set the Error Variance of RET4_3 Free

Set the Error Variance of RET5_3 Free

Set the Variance of ET_1 Free

Set the Variance of ET_2 Free

Set the Variance of ET_3 Free

Set the Covariance between ET_1 and ET_2 Free

Set the Covariance between ET_2 and ET_3 Free

Set the Covariance between ET_1 and ET_3 Free

Path Diagram

End of Problem

\section{The Estimation of $\mathrm{M3}$}

\section{!ET M3}

Observed Variables

ET1_1 ET2_1 ET3_1 RET4_1 RET5_1 ET1_2 ET2_2 ET3_2 RET4_2 RET5_2

ET1_3 ET2_3 ET3_3 RET4_3 RET5_3

Raw Data from File ET.psf

Sample Size 63

Latent Variables

ET_1ET_2ET_3

Equations

ET1_1 $=2.67 *$ CONST $+1 *$ ET_1

ET2 $1=2.78 * \mathrm{CONST}+0.97 * \mathrm{ET} 11$

ET3_1 $=2.53 *$ CONST $+0.97 *$ ET_1

RET4_1 $=3.54 *$ CONST $+0.67 *$ ET 1

RET5_1 $=2.69 * \mathrm{CONST}+0.58 * \mathrm{ET} \_1$ 
ET1_2 $=2.67 *$ CONST $+1 *$ ET_2

ET2_2 $=2.78 *$ CONST $+0.97 *$ ET_2

ET3_2 $=2.53 *$ CONST $+0.97 *$ ET_2

RET4_2 $=3.54 *$ CONST $+0.67 *$ ET_2

RET5_2 $=2.69 *$ CONST $+0.58 *$ ET_2

ET1_3 $=2.67 *$ CONST $+1 *$ ET_3

ET2_3 $=2.78 *$ CONST $+0.97 *$ ET_3

ET3_3 $=2.53 *$ CONST $+0.97 *$ ET_3

RET4_3 $=3.54 *$ CONST $+0.67 *$ ET_3

RET5_3 $=2.69 *$ CONST $+0.58 *$ ET_3

Let the Errors between ET1_1 and ET1_2 Correlate Let the Errors between ET1_2 and ET1_3 Correlate Let the Errors between ET1_1 and ET1_3 Correlate Let the Errors between ET2_1 and ET2_2 Correlate Let the Errors between ET2_2 and ET2_3 Correlate Let the Errors between ET2_1 and ET2_3 Correlate Let the Errors between ET3_1 and ET3_2 Correlate Let the Errors between ET3_2 and ET3_3 Correlate Let the Errors between ET3_1 and ET3_3 Correlate Let the Errors between RET4_1 and RET4_2 Correlate Let the Errors between RET4_2 and RET4_3 Correlate Let the Errors between RET4_1 and RET4_3 Correlate Let the Errors between RET5_1 and RET5_2 Correlate Let the Errors between RET5_2 and RET5_3 Correlate Let the Errors between RET5_1 and RET5_3 Correlate

Set the Error Variance of ET1_1 Free Set the Error Variance of ET2_1 Free

Set the Error Variance of ET3_ 1 Free

Set the Error Variance of RET4_1 Free

Set the Error Variance of RET5_1 Free

Set the Error Variance of ET1_2 Free

Set the Error Variance of ET2_2 Free

Set the Error Variance of ET3_ 2 Free

Set the Error Variance of RET4_2 Free

Set the Error Variance of RET5_2 Free

Set the Error Variance of ET1_3 Free

Set the Error Variance of ET2 3 Free

Set the Error Variance of ET3_ 3 Free 
Set the Error Variance of RET4_3 Free

Set the Error Variance of RET5_3 Free

Set the Variance of ET_1 Free

Set the Variance of ET_2 Free

Set the Variance of ET_3 Free

Set the Covariance between ET_1 and ET_2 Free

Set the Covariance between ET_2 and ET_3 Free

Set the Covariance between ET_1 and ET_3 Free

Path Diagram

End of Problem

\section{The Estimation of M4}

!ET M4

Observed Variables

ET1_1 ET2_1 ET3_1 RET4_1 RET5_1 ET1_2 ET2_2 ET3_2 RET4_2 RET5_2

ET1_3 ET2_3 ET3_3 RET4_3 RET5_3

Raw Data from File ET.psf

Sample Size 63

Latent Variables

ET_1 ET_2 ET_3

Equations

ET1_1 $=2.67 *$ CONST $+1 *$ ET $\_1$

ET2 $1=2.78 * \mathrm{CONST}+0.97 * \mathrm{ET} 1$

ET3_1 $=2.53 *$ CONST $+0.97 *$ ET_1

RET4_1 $=3.54 *$ CONST $+0.67 *$ ET_1

RET5_1 $=2.69 *$ CONST $+0.58 *$ ET_1

ET1_2 $=2.67 *$ CONST $+1 *$ ET $\_2$

ET2 $2=2.78 * \mathrm{CONST}+0.97 * \mathrm{ET} 2$

ET3_2 $=2.53 *$ CONST $+0.97 *$ ET_2

RET4 $2=3.54 *$ CONST $+0.67 *$ ET 2

RET5_2 $=2.69 *$ CONST $+0.58 *$ ET_2

ET1_3 $=2.67 *$ CONST $+1 *$ ET 33

ET2 $3=2.78 * \mathrm{CONST}+0.97 * \mathrm{ET} 3$

ET3_3 $=2.53 *$ CONST $+0.97 *$ ET_3 
RET4_3 $=3.54 *$ CONST $+0.67 *$ ET_3

RET5_3 $=2.69 * \mathrm{CONST}+0.58 * \mathrm{ET}_{-} 3$

Let the Errors between ET1_1 and ET1_2 Correlate Let the Errors between ET1_2 and ET1_3 Correlate Let the Errors between ET1_1 and ET1_3 Correlate Let the Errors between ET2_1 and ET2_2 Correlate Let the Errors between ET2_2 and ET2_3 Correlate Let the Errors between ET2_1 and ET2_3 Correlate Let the Errors between ET3_1 and ET3_2 Correlate Let the Errors between ET3_2 and ET3_3 Correlate Let the Errors between ET3_1 and ET3_3 Correlate Let the Errors between RET4_1 and RET4_2 Correlate Let the Errors between RET4_2 and RET4_3 Correlate Let the Errors between RET4_1 and RET4_3 Correlate Let the Errors between RET5_1 and RET5_2 Correlate Let the Errors between RET5_2 and RET5_3 Correlate Let the Errors between RET5_1 and RET5_3 Correlate

Equal Error Variances: ET1_1 ET1_2 ET1_3

Equal Error Variances: ET2_1 ET2_2 ET2_3

Equal Error Variances: ET3_1 ET3_2 ET3_3

Equal Error Variances: RET4_1 RET4_2 RET4_3

Equal Error Variances: RET5_1 RET5_2 RET5_3

Set the Variance of ET_1 Free

Set the Variance of ET_2 Free

Set the Variance of ET_3 Free

Set the Covariance between ET_1 and ET_2 Free

Set the Covariance between ET_2 and ET_3 Free

Set the Covariance between ET_1 and ET_3 Free

Path Diagram

End of Problem

\section{The Estimation of M5}

!ET M5

Observed Variables

ET1_1 ET2_1 ET3_1 RET4_1 RET5_1 ET1_2 ET2_2 ET3_2 RET4_2 RET5_2

ET1_3 ET2_3 ET3_3 RET4_3 RET5_3 
Raw Data from File ET.psf

Sample Size 63

Latent Variables

ET_1ET_2 ET_3

Equations

ET1_1 $=2.67 * \mathrm{CONST}+1 * \mathrm{ET} \_1$

ET2_1 $=2.78 * \mathrm{CONST}+0.97 * \overline{\mathrm{ET}} \_1$

ET3_1 $=2.53 * \mathrm{CONST}+0.97 * \mathrm{ET} \_1$

RET4_1 $=3.54 * \mathrm{CONST}+0.67 * \mathrm{ET} \_1$

RET5_1 $=2.69 * \mathrm{CONST}+0.58 * \mathrm{ET}_{-} 1$

ET1_2 $=2.67 * \mathrm{CONST}+1 * \mathrm{ET} \_2$

ET2_2 $=2.78 * \mathrm{CONST}+0.97 * \mathrm{ET} \_2$

ET3_2 $=2.53 * \mathrm{CONST}+0.97 * \mathrm{ET} \_2$

RET4_2 $=3.54 * \mathrm{CONST}+0.67 * \mathrm{ET} \_2$

RET5_2 $=2.69 * \mathrm{CONST}+0.58 * \mathrm{ET} \_2$

ET1_3 $=2.67 * \mathrm{CONST}+1 * \mathrm{ET} \_3$

ET2_3 $=2.78 * \mathrm{CONST}+0.97 * \mathrm{ET} \_3$

ET3_3 $=2.53 * \mathrm{CONST}+0.97 * \mathrm{ET} \_3$

RET4_3 $=3.54 * \mathrm{CONST}+0.67 * \mathrm{ET} \_3$

RET5_3 $=2.69 *$ CONST $+0.58 * E T \_3$

Let the Errors between ET1_1 and ET1_2 Correlate Let the Errors between ET1_2 and ET1_3 Correlate Let the Errors between ET1_1 and ET1_3 Correlate Let the Errors between ET2_1 and ET2_2 Correlate Let the Errors between ET2_2 and ET2_3 Correlate Let the Errors between ET2_1 and ET2_3 Correlate Let the Errors between ET3_1 and ET3_2 Correlate Let the Errors between ET3_2 and ET3_3 Correlate Let the Errors between ET3_1 and ET3_3 Correlate Let the Errors between RET4_1 and RET4_2 Correlate Let the Errors between RET4_2 and RET4_3 Correlate Let the Errors between RET4_1 and RET4_3 Correlate Let the Errors between RET5_1 and RET5_2 Correlate Let the Errors between RET5_2 and RET5_3 Correlate Let the Errors between RET5_1 and RET5_3 Correlate

Equal Error Variances: ET1_1 ET1_2 ET1_3 
Equal Error Variances: ET2_1 ET2_2 ET2_3

Equal Error Variances: ET3_1 ET3_2 ET3_3

Equal Error Variances: RET4_1 RET4_2 RET4_3

Equal Error Variances: RET5_1 RET5_2 RET5_3

Equal Variances: ET_1 ET_2 ET_3

Set the Covariance between ET_1 and ET_2 Free

Set the Covariance between ET_2 and ET_3 Free

Set the Covariance between ET_1 and ET_3 Free

Path Diagram

End of Problem

\section{The Estimation of M6}

!ET M6

Observed Variables

ET1_1 ET2_1 ET3_1 RET4_1 RET5_1 ET1_2 ET2_2 ET3_2 RET4_2 RET5_2

ET1_3 ET2_3 ET3_3 RET4_3 RET5_3

Raw Data from File ET.psf

Sample Size 63

Latent Variables

ET_1 ET_2ET_3

Equations

ET1_1 $=2.67 *$ CONST $+1 *$ ET $\_1$

ET2 $1=2.78 * \mathrm{CONST}+0.97 * \mathrm{ET} \_1$

ET3_1 $=2.53 *$ CONST $+0.97 *$ ET_1

RET4_1 $=3.54 *$ CONST $+0.67 *$ ET_1

RET5_ $1=2.69 * \mathrm{CONST}+0.58 * \mathrm{ET} \_1$

ET1_2 $=2.67 *$ CONST $+1 *$ ET 22

ET2 $2=2.78 * \mathrm{CONST}+0.97 * \mathrm{ET} 2$

ET3_2 $=2.53 *$ CONST $+0.97 *$ ET_2

RET4_2 $=3.54 *$ CONST $+0.67 *$ ET_2

RET5_2 $=2.69 * \mathrm{CONST}+0.58 * \mathrm{ET} \_2$

ET1_3 $=2.67 *$ CONST $+1 *$ ET_3

ET2 $3=2.78 * \mathrm{CONST}+0.97 * \mathrm{ET} 3$

ET3_3 $=2.53 *$ CONST $+0.97 *$ ET_3 
RET4_3 $=3.54 *$ CONST $+0.67 *$ ET_3

RET5_3 $=2.69 * \mathrm{CONST}+0.58 * \mathrm{ET}_{-} 3$

Let the Errors between ET1_1 and ET1_2 Correlate Let the Errors between ET1_2 and ET1_3 Correlate Let the Errors between ET1_1 and ET1_3 Correlate Let the Errors between ET2_1 and ET2_2 Correlate Let the Errors between ET2_2 and ET2_3 Correlate Let the Errors between ET2_1 and ET2_3 Correlate Let the Errors between ET3_1 and ET3_2 Correlate Let the Errors between ET3_2 and ET3_3 Correlate Let the Errors between ET3_1 and ET3_3 Correlate Let the Errors between RET4_1 and RET4_2 Correlate Let the Errors between RET4_2 and RET4_3 Correlate Let the Errors between RET4_1 and RET4_3 Correlate Let the Errors between RET5_1 and RET5_2 Correlate Let the Errors between RET5_2 and RET5_3 Correlate Let the Errors between RET5_1 and RET5_3 Correlate

Equal Error Variances: ET1_1 ET1_2 ET1_3

Equal Error Variances: ET2_1 ET2_2 ET2_3

Equal Error Variances: ET3_1 ET3_2 ET3_3

Equal Error Variances: RET4_1 RET4_2 RET4_3

Equal Error Variances: RET5_1 RET5_2 RET5_3

Equal Variances: ET_1 ET_2 ET_3

Set the Covariance between ET_1 and ET_2 to 0.82

Set the Covariance between ET_2 and ET_3 to 0.82

Set the Covariance between ET_1 and ET_3 to 0.82

Path Diagram

End of Problem

\section{The Estimation of $\mathrm{M7}$}

!ET M7

Observed Variables

ET1_1 ET2_1 ET3_1 RET4_1 RET5_1 ET1_2 ET2_2 ET3_2 RET4_2 RET5_2

ET1_3 ET2_3 ET3_3 RET4_3 RET5_3

Raw Data from File ET.psf

Sample Size 63 
Latent Variables

ET_1ET_2ET_3

Equations

ET1_1 $=2.67 * \mathrm{CONST}+1 * \mathrm{ET}_{-} 1$

ET2_1 $=2.78 * \mathrm{CONST}+0.97 * \mathrm{ET} \_1$

ET3_1 $=2.53 * \mathrm{CONST}+0.97 * \mathrm{ET} \_1$

RET4_1 $=3.54 * \mathrm{CONST}+0.67 * \mathrm{ET}_{-} 1$

RET5_1 $=2.69 * \mathrm{CONST}+0.58 * \mathrm{ET}_{-} 1$

ET1_2 $=2.67 * \mathrm{CONST}+1 * \mathrm{ET} \_2$

ET2 $22=2.78 * \mathrm{CONST}+0.97 * \mathrm{ET} \_2$

ET3_2 $=2.53 * \mathrm{CONST}+0.97 * \mathrm{ET} \_2$

RET4_2 $=3.54 * \mathrm{CONST}+0.67 * \mathrm{ET} \_2$

RET5_2 $=2.69 * \mathrm{CONST}+0.58 * \mathrm{ET} \_2$

ET1_3 $=2.67 * \mathrm{CONST}+1 * \mathrm{ET} \_3$

ET2 $3=2.78 * \mathrm{CONST}+0.97 * \mathrm{ET} \_3$

ET3_3 $=2.53 * \mathrm{CONST}+0.97 * \mathrm{ET} \_3$

RET4_3 $=3.54 * \mathrm{CONST}+0.67 * \mathrm{ET} 33$

RET5_3 $=2.69 *$ CONST $+0.58 * E T \_3$

ET_ $1=0.0017 * \mathrm{CONST}$

ET_2 $=0.0017 * \mathrm{CONST}$

ET_3 $=0.0017 * \mathrm{CONST}$

Let the Errors between ET1_1 and ET1_2 Correlate Let the Errors between ET1_2 and ET1_3 Correlate Let the Errors between ET1_1 and ET1_3 Correlate Let the Errors between ET2_1 and ET2_2 Correlate Let the Errors between ET2_2 and ET2_3 Correlate Let the Errors between ET2_1 and ET2_3 Correlate Let the Errors between ET3_1 and ET3_2 Correlate Let the Errors between ET3_2 and ET3_3 Correlate Let the Errors between ET3_1 and ET3_3 Correlate Let the Errors between RET4_1 and RET4_2 Correlate Let the Errors between RET4_2 and RET4_3 Correlate Let the Errors between RET4_1 and RET4_3 Correlate Let the Errors between RET5_1 and RET5_2 Correlate Let the Errors between RET5_2 and RET5_3 Correlate Let the Errors between RET5_1 and RET5_3 Correlate 
Equal Error Variances: ET1_1 ET1_2 ET1_3

Equal Error Variances: ET2_1 ET2_2 ET2_3

Equal Error Variances: ET3_1 ET3_2 ET3_3

Equal Error Variances: RET4_1 RET4_2 RET4_3

Equal Error Variances: RET5_1 RET5_2 RET5_3

Equal Variances: ET_1 ET_2 ET_3

Set the Covariance between ET_1 and ET_2 to 0.82

Set the Covariance between ET_2 and ET_3 to 0.82

Set the Covariance between ET_1 and ET_3 to 0.82

Path Diagram

End of Problem

\section{LISREL Syntax Chapter 3}

\section{The Estimation of M1}

!ET M1

Group1

Observed Variables

ET1_1 ET2_1 ET3_1 RET4_1 RET5_1 ET1_2ET2_2ET3_2 RET4_2 RET5_2

Raw Data from File ET.psf

Sample Size 52

Latent Variables

ET_1 ET_2

Equations

ET1_1 $=$ CONST $+1 * E T \_1$

$\mathrm{ET} 2 \_1=\mathrm{CONST}+\mathrm{ET} \_1$

ET3_1 $=$ CONST+ET_1

RET4_1=CONST+ET_1

RET5_1=CONST+ET_1

ET1_2 $=$ CONST $+1 *$ ET_ 2

ET2_2 $=$ CONST + ET_2

ET3_2 $=$ CONST+ET_2

RET4_2 $=$ CONST + ET 2

RET5_2=CONST+ET_2 
Let the Errors between ET1_1 and ET1_2 Correlate Let the Errors between ET2_1 and ET2_2 Correlate

Let the Errors between ET3_1 and ET3_2 Correlate Let the Errors between RET4_1 and RET4_2 Correlate

Let the Errors between RET5_1 and RET5_2 Correlate

Set the Error Variance of ET1_1 Free

Set the Error Variance of ET2_1 Free

Set the Error Variance of ET3_1 Free

Set the Error Variance of RET4_1 Free

Set the Error Variance of RET5_1 Free

Set the Error Variance of ET1_1 Free

Set the Error Variance of ET2_1 Free

Set the Error Variance of ET3_1 Free

Set the Error Variance of RET4_1 Free

Set the Error Variance of RET5_1 Free

Set the Variance of ET_1 Free

Set the Variance of ET_2 Free

Set the Covariance between ET_1 and ET_2 Free

Group2

Raw Data from File ET2.psf

Sample Size 49

Path Diagram

End of Problem

\section{The Estimation of M2}

!ET M2

Observed Variables

ET1_1 ET2_1 ET3_1 RET4_1 RET5_1 ET1_2ET2_2 ET3_2 RET4_2 RET5_2

Raw Data from File ET.psf

Sample Size 52

Latent Variables

ET_1ET_2

Equations 
ET1_1=CONST $+1 * E T \_1$

ET2_1 $=\mathrm{CONST}+1.02 * \mathrm{ET} \_1$

ET3_1=CONST $+1.14 *$ ET_1

RET4_1 $=$ CONST $+0.74 * E T \_1$

RET5_1 $=$ CONST $+0.65 *$ ET_1

ET1_2 $=$ CONST $+1 *$ ET_2

ET2 $2=\mathrm{CONST}+1.02 * \mathrm{ET} 2$

ET3_2 $=$ CONST $+1.14 * E T \_2$

RET4 $2=$ CONST $+0.74 *$ ET 2

RET5_2 $=$ CONST $+0.65 *$ ET_2

Let the Errors of ET1_1 and ET1_2 Correlate

Let the Errors of ET2_1 and ET2_2 Correlate

Let the Errors of ET3_1 and ET3_2 Correlate

Let the Errors of RET4_1 and RET4_2 Correlate

Let the Errors of RET5_1 and RET5_2 Correlate

Set the Error Variance of ET1_1 Free

Set the Error Variance of ET2_1 Free

Set the Error Variance of ET3_1 Free

Set the Error Variance of RET4 1 Free

Set the Error Variance of RET5_1 Free

Set the Error Variance of ET1_1 Free

Set the Error Variance of ET2_1 Free

Set the Error Variance of ET3_1 Free

Set the Error Variance of RET4_1 Free

Set the Error Variance of RET5_1 Free

Set the Variance of ET_1 Free

Set the Variance of ET_2 Free

Set the Covariance between ET_1 and ET_2 Free

Group2

Raw Data from File ET2.psf

Sample Size 49

Path Diagram

End of Problem 


\section{The Estimation of M3}

\section{!ET M3}

Observed Variables

ET1_1 ET2_1 ET3_1 RET4_1 RET5_1 ET1_2 ET2_2 ET3_2 RET4_2 RET5_2

Raw Data from File ET.psf

Sample Size 52

Latent Variables

ET_1 ET_2

Equations

$$
\begin{aligned}
& \text { ET1_1 } 2.73 * \mathrm{CONST}+1 * \mathrm{ET} \_1 \\
& \mathrm{ET} 2 \_1=2.75 * \mathrm{CONST}+1.02 * \mathrm{ET} \_1 \\
& \text { ET3_1 }=2.67 * \mathrm{CONST}+1.14 * \mathrm{ET} 1 \\
& \text { RET4_1 }=3.47 * \mathrm{CONST}+0.74 * \mathrm{ET} 11 \\
& \text { RET5_1 }=2.72 * \mathrm{CONST}+0.65 * \mathrm{ET} \_1 \\
& \text { ET1_2 }=2.73 * \mathrm{CONST}+1 * \mathrm{ET} 2 \\
& \text { ET2_2 }=2.75 * \mathrm{CONST}+1.02 * \mathrm{ET} 2 \\
& \text { ET3_2 }=2.67 * \mathrm{CONST}+1.14 * \mathrm{ET} 2 \\
& \text { RET4_2 }=3.47 * \mathrm{CONST}+0.74 * \mathrm{ET} 22 \\
& \text { RET5_2 }=2.72 * \mathrm{CONST}+0.65 * \mathrm{ET} \_2
\end{aligned}
$$

Let the Errors of ET1_1 and ET1_2 Correlate

Let the Errors of ET2_1 and ET2_2 Correlate

Let the Errors of ET3_1 and ET3_2 Correlate

Let the Errors of RET4_1 and RET4_2 Correlate

Let the Errors of RET5_1 and RET5_2 Correlate

Set the Error Variance of ET1_1 Free

Set the Error Variance of ET2_1 Free

Set the Error Variance of ET3_1 Free

Set the Error Variance of RET4_1 Free

Set the Error Variance of RET5_1 Free

Set the Error Variance of ET1_1 Free

Set the Error Variance of ET2_1 Free

Set the Error Variance of ET3_1 Free

Set the Error Variance of RET4_1 Free

Set the Error Variance of RET5_1 Free

Set the Variance of ET_1 Free 
Set the Variance of ET_2 Free

Set the Covariance between ET_1 and ET_2 Free

Group2

Raw Data from File ET2.psf

Sample Size 49

Path Diagram

End of Problem

\section{The Estimation of M4}

!ET M4

Observed Variables

ET1_1 ET2_1 ET3_1 RET4_1 RET5_1 ET1_2 ET2_2 ET3_2 RET4_2 RET5_2

Raw Data from File ET.psf

Sample Size 52

Latent Variables

ET_1ET_2

Equations

ET1_1 $=2.73 *$ CONST $+1 *$ ET_1

ET2 $1=2.75 * \mathrm{CONST}+1.02 * \mathrm{ET}$ 1

ET3_1 $=2.67 *$ CONST $+1.14 *$ ET_1

RET4_1 $=3.47 *$ CONST $+0.74 *$ ET_1

RET5_ $1=2.72 *$ CONST $+0.65 *$ ET_ 1

ET1_2 $=2.73 *$ CONST $+1 *$ ET_2

ET2 $2=2.75 * \mathrm{CONST}+1.02 * \mathrm{ET} \_2$

ET3_2 $=2.67 * \mathrm{CONST}+1.14 * \mathrm{ET} \_2$

RET4_2 $=3.47 *$ CONST $+0.74 *$ ET_2

RET5_2 $=2.72 *$ CONST $+0.65 *$ ET_2

Let the Errors of ET1_1 and ET1_2 Correlate

Let the Errors of ET2_1 and ET2_2 Correlate

Let the Errors of ET3_1 and ET3_2 Correlate

Let the Errors of RET4_1 and RET4_2 Correlate

Let the Errors of RET5_1 and RET5_2 Correlate

Equal Error Variances: ET1_1 ET1_2 
Equal Error Variances: ET2_1 ET2_2

Equal Error Variances: ET3_1 ET3_2

Equal Error Variances: RET4_1 RET4_2

Equal Error Variances: RET5_1 RET5_2

Set the Variance of ET_1 Free

Set the Variance of ET_2 Free

Set the Covariance between ET_1 and ET_2 Free

Group2

Raw Data from File ET2.psf

Sample Size 49

Path Diagram

End of Problem

\section{The Estimation of M5}

!ET M5

Observed Variables

ET1_1 ET2_1 ET3_1 RET4_1 RET5_1 ET1_2 ET2_2 ET3_2 RET4_2 RET5_2

Raw Data from File ET.psf

Sample Size 52

Latent Variables

ET_1 ET_2

Equations

ET1_1 $=2.73 *$ CONST $+1 *$ ET $\_1$

ET2 $1=2.75 * \mathrm{CONST}+1.02 * \mathrm{ET} \_1$

ET3_1 $=2.67 *$ CONST $+1.14 * E T \_1$

RET4_1 $=3.47 *$ CONST $+0.74 * E T \_1$

RET5_ $1=2.72 *$ CONST $+0.65 *$ ET_ 1

ET1_2 $=2.73 *$ CONST $+1 *$ ET $\_2$

ET2 $2=2.75 * \mathrm{CONST}+1.02 * \mathrm{ET} 2$

ET3_2 $=2.67 * \mathrm{CONST}+1.14 * \mathrm{ET} \_2$

RET4_2 $=3.47 *$ CONST $+0.74 *$ ET_2

RET5_2 $=2.72 * \mathrm{CONST}+0.65 * \mathrm{ET} \_2$

Let the Errors of ET1_1 and ET1_2 Correlate 
Let the Errors of ET2_1 and ET2_2 Correlate

Let the Errors of ET3_1 and ET3_2 Correlate

Let the Errors of RET4_1 and RET4_2 Correlate

Let the Errors of RET5_1 and RET5_2 Correlate

Equal Error Variances: ET1_1 ET1_2

Equal Error Variances: ET2_1 ET2_2

Equal Error Variances: ET3_1 ET3_2

Equal Error Variances: RET4_1 RET4_2

Equal Error Variances: RET5_1 RET5_2

Equal Variances ET_1 ET_2

Set the Covariance between ET_1 and ET_2 Free

Group2

Raw Data from File ET2.psf

Sample Size 49

Path Diagram

End of Problem

\section{The Estimation of M6}

!ET M6

Observed Variables

ET1_1 ET2_1 ET3_1 RET4_1 RET5_1 ET1_2 ET2_2ET3_2 RET4_2 RET5_2

Raw Data from File ET.psf

Sample Size 52

Latent Variables

ET_1 ET_2

Equations

ET1_1 $=2.73 *$ CONST $+1 *$ ET_1

ET2_1 $=2.75 * \mathrm{CONST}+1.02 * \mathrm{ET} \_1$

ET3_1 $=2.67 *$ CONST $+1.14 *$ ET_1

RET4_1 $=3.47 *$ CONST $+0.74 * E T \_1$

RET5_1 $=2.72 * \mathrm{CONST}+0.65 * \mathrm{ET}_{-} 1$

ET1_2 $=2.73 *$ CONST $+1 *$ ET_2 
ET2_2 $=2.75 * \mathrm{CONST}+1.02 * \mathrm{ET}$ 2

ET3_2 $=2.67 *$ CONST $+1.14 *$ ET_2

RET4_2 $=3.47 *$ CONST $+0.74 *$ ET_2

RET5_2 $=2.72 *$ CONST $+0.65 *$ ET_2

Let the Errors of ET1_1 and ET1_2 Correlate

Let the Errors of ET2_1 and ET2_2 Correlate

Let the Errors of ET3_1 and ET3_2 Correlate

Let the Errors of RET4_1 and RET4_2 Correlate

Let the Errors of RET5_1 and RET5_2 Correlate

Equal Error Variances: ET1_1 ET1_2

Equal Error Variances: ET2_1 ET2_2

Equal Error Variances: ET3_1 ET3_2

Equal Error Variances: RET4_1 RET4_2

Equal Error Variances: RET5_1 RET5_2

Equal Variances ET_1 ET_2

Set the Covariance between ET_1 and ET_2 to 0.9

Group2

Raw Data from File ET2.psf

Sample Size 49

Path Diagram

End of Problem

\section{The Estimation of $\mathrm{M7}$}

!ET M7

Observed Variables

ET1_1ET2_1 ET3_1 RET4_1 RET5_1ET1_2 ET2_2 ET3_2 RET4_2 RET5_2

Raw Data from File ET.psf

Sample Size 52

Latent Variables

ET_1 ET_2

Equations

ET1 $1=2.73 *$ CONST $+1 *$ ET 11

ET2_ $1=2.75 *$ CONST $+1.02 *$ ET_1 
ET3_1 $=2.67 *$ CONST $+1.14 *$ ET_1

RET4_1 $=3.47 * \mathrm{CONST}+0.74 * \mathrm{ET}$ -

RET5_1 $=2.72 *$ CONST $+0.65 *$ ET_1

ET1_2 $=2.73 *$ CONST $+1 *$ ET_2

ET2 $22=2.75 * \mathrm{CONST}+1.02 * \mathrm{ET} \_2$

ET3_2 $=2.67 * \mathrm{CONST}+1.14 * \mathrm{ET} \_2$

RET4_2 $=3.47 *$ CONST $+0.74 *$ ET_2

RET5_2 $=2.72 *$ CONST $+0.65 *$ ET_2

ET_1 $=-0.1262 *$ CONST

ET $2=-0.1262 *$ CONST

Let the Errors of ET1_1 and ET1_2 Correlate

Let the Errors of ET2_1 and ET2_2 Correlate

Let the Errors of ET3_1 and ET3_2 Correlate

Let the Errors of RET4_1 and RET4_2 Correlate

Let the Errors of RET5_1 and RET5_2 Correlate

Equal Error Variances: ET1_1 ET1_2

Equal Error Variances: ET2_1 ET2_2

Equal Error Variances: ET3_1 ET3_2

Equal Error Variances: RET4_1 RET4_2

Equal Error Variances: RET5_1 RET5_2

Equal Variances ET_1 ET_2

Set the Covariance between ET_1 and ET_2 to 0.9

Group2

Raw Data from File ET2.psf

Sample Size 49

Path Diagram

End of Problem 


\section{Appendix C The Content of the Computer Simulation}

\section{Scenario 1}

\section{Information introduced in video}

Susan: Good Morning, Tom!

Tom: Good Morning, Susan!

Susan: How are you?

Tom: Fine! And you?

Susan: Couldn't be better! Except for yesterday, there was this angry guy calling up our customer service line and reporting a fire incident related to his Martha@Home coffeemaker, an M10 model!

Tom: Oh, what happened?

Susan: He said that he was just making coffee in the kitchen, as he always did every morning. He started the machine and then went to the shower. When he came out the bathroom 10 minutes later, the whole coffee machine was on fire!

Tom: Oh ... !

Susan: He was very angry, saying if he had had come out of shower one minute later, the entire house would have been burned down! Well, I feel sorry for him, but it is really not my business! Why yelling at me!

Tom: Sorry to hear that, Susie!

Susan: But it is indeed very strange, because we didn't have so many complaints about the M10 model before and this one is already the third fire-related complaint we have received in Seattle this year!

Tom: ... . . . .

\section{Computer-Participant interface}

Martha@Home Co. is a Seattle-based company that produces small electronic kitchenware, such as water boiler, coffeemaker, blender, toaster, etc. Two days ago, someone called the company's toll-free customer-service line complaining a fire incident related to a Martha@Home M10 coffeemaker, one of the company's most successful products nationwide. As the CEO of Martha@ Home Co., what are you going to do after overhearing the conversation of two employees in the cafeteria?

A. Put it aside, since the other fire incidents reported this year were all caused by the customers' inappropriate use of the machine.

B. Consider it a serious incident and decide to have an emergent senior-manager meeting to discuss what the company should do to cope with a possible crisis situation in the future.

C. Call customer service department and product development department and ask them to gather more information concerning this fire incident. 


\section{Scenario 2}

\section{Information introduced in video}

Marketing Manager: Good morning! Here is the report about the launching campaign of the new Martha@Home Kitchen Assistant Set that we discussed yesterday. And, by the way, have you read today's Seattle Times? A house on $29^{\text {th }}$ Avenue South caught on fire yesterday. The police suspect it might have something to do with an electric coffeemaker the house owner bought from us half a year ago . . . an M10 model, if I remember correctly. The house was burned downed and four people were injured and sent to hospital including a boy of four years old and a girl of six years old. Now the whole company are talking about the news ...

\section{Computer-Participant interface}

Very soon, The Seattle Times, a major local newspaper, covered a household fire that the police suspected to be caused by a Martha@Home coffeemaker. Four persons were injured and hospitalized, including two kids aged 4 and 6 . The fire incident has immediately become a "hot" topic in town, especially within the company. You decide

A. To immediately have a press release, apologizing to the victims of the fire incident and promising that Martha@Home would try all the best to improve product quality and prevent similar incidents from happening.

B. To wait for more information from the police and cooperate if they come to the company to investigate, but not initiate a press release.

C. To call upon a senior-manager meeting, suggesting setting up an ad-hoc team to thoroughly investigate the quality, design, and production of Martha@Home M10 coffeemakers, and to send emails to employees, informing them of the company's reactions to the fire incident.

\section{Scenario 3}

\section{Information introduced in video}

Personal Assistant: Good morning! Michael Schneider, the head of the R\&D department wants to meet you now. It is about the M10 Martha@Home coffeemaker.

Michael Schneider: Good morning! We just had the safety test report of the M10 coffeemakers. We found that when the environment temperature is approaching 100 Fahrenheit (37.8 Celsius), this plastic component (show it) inside the machine will catch on fire. Although we have already had a fire-protection device inside the machine, it doesn't prevent the whole machine from burning when this plastic piece starts to burn... 


\section{Computer-Participant interface}

The safety test results from the $R \& D$ department show that when the environment temperature rises above $100 \mathrm{~F}(37.8 \mathrm{C})$, an internal plastic component of a Martha@Home M10 coffeemaker becomes likely to ignite, despite a built-in fire protection device. After realizing such quality deficiency of the M10 model, you think the company should

A. Voluntarily recall all defective coffeemakers nationwide, given a life-threatening consequence of the deficiency, and communicate the recalling action to the public and all stakeholders of the company.

B. Not immediately launch a nationwide recall, since there has not been solid evidence from the police that it was the Martha@Home M10 coffeemaker that caused the two fire incidents. In addition, it is very costly to launch a nationwide recall.

C. Voluntarily recall all defective coffeemakers nationwide, given a life-threatening consequence of the product deficiency, and only communicate the recalling action to the relevant customers, retailers, and distributors but not to the public or other "non-involved" stakeholders.

\section{Scenario 4}

\section{Information introduced in texts/ Computer-Participant interface}

Finally, the board of directors and top managers agree to launch a nationwide recall of Martha@Home M10 coffeemakers. Emails are sent to retailers, distributors, business partners, and internal employees; and letters are sent to each customer with a product registration card of an M10 model. A special customer service hotline is also set up. About $10 \%$ of the sold defective coffeemakers are returned within two weeks. Now, you think the company should

A. Wait for another two weeks to see how many more coffeemakers are returned and try to maintain the company's operation as usual as before.

B. Put more efforts into the recalling, initiate a large-scale public relation campaign to inform as many customers as possible and shut down the production division for all Martha@Home coffeemakers.

C. Keep tracking down more customers of the M10 coffeemakers by using additional databases of customers who bought other Martha@Home kitchen products and keep producing coffeemakers that do not contain the defective plastic components.

\section{Scenario 5}

\section{Information introduced in video}

Personal Assistant: Good morning! Here is the update about the M10 coffeemaker recalling. Until yesterday, the return rate of the defective coffeemakers has reached $68 \%$ nationwide. However, not a single coffeemaker has been returned from Wyo- 
ming, Idaho, Nevada, Utah, and Colorado, even though the M10 model is not a best seller in these states, as in the other states ...

\section{Computer-Participant interface}

Two weeks later, $68 \%$ of the defective M10 coffeemakers are returned. You notice that five middle-west states - Wyoming, Idaho, Nevada, Utah, and Colorado - have not returned any M10 coffeemaker yet, even though the M10 model is not as popular in these states as in the rest of the country. You think now the company should

A. Send representatives to the five states to recall the defective M10 coffeemakers from retailers and distributors, and track down possible customers of the M10 model, replacing the purchased coffeemakers or refunding the customers.

B. Terminate the recalling at this point, since the majority of the defective units have already been returned and it is not worth the costs sending representatives to the five states to recall the remaining defective units.

C. Estimate the costs for sending representatives to the five states, compare the estimated costs with the revenues made from the sold M10 coffeemakers in these states, and then decide whether to send representatives or not.

\section{Scenario 6}

\section{Information introduced in video}

Personal Assistant: Good afternoon! Jack Smith, the head of the legal department, is here to meet with you.

Jack Smith: Good afternoon! It has been a busy period for our department and the personnel department. We have been investigating the deal that we made with Priority Inc., the current supplier for that inflammable plastic piece inside the M-10 model, about one year ago. It turned out that, Tom Robinson, our procurement manager, had had 37 fancy meals with the sales representatives from Priority Inc. in the most extravagant restaurants in town. Priority Inc. explained that the meals were to build a "healthy reciprocal" business relationship with us. Although having "free meals" with business partners is not considered as violating the company's ethic codes, but ...

\section{Computer-Participant interface}

An investigation team from the company's legal department reported that one year ago, when Martha@Home Co. decided to switch the supplier of the "unexpectedly ignitable" plastic component, the procurement manager accepted a number of luxurious meals in the most extravagant restaurants in Seattle, which were all paid by the current supplier in order to establish a "healthy and reciprocal" business relationship with Marth@Home Co. Although Martha@Home Co. does not explicitly 
prohibit the procurement personnel from receiving "free meals" from its suppliers, you think the company should

A. Immediately fire the procurement manager, change the current supplier of the plastic component, and inform the public and municipal legal authorities of the potential connections of the procurement manager's misconducts, the defective plastic component, and the earlier reported house fire.

B. Immediately fire the procurement manager, change the current supplier of the plastic component, but not inform any external parties of the results of the internal investigation in order to avoid potential public concern on the company's ethic codes.

C. Gather more information about what exactly happened when the procurement manager decided to give the contract to the current supplier of the plastic component in order to judge whether the person has violated the company's ethic codes or not.

\section{Scenario 7}

\section{Information introduced in video}

Jack Smith: Hi, this is Jack Smith calling from the legal department. You'd better turn the TV on. There is something very important to us!

( $\mathrm{TV}$ is turned on)

Host: Good evening, everyone! Welcome to today's Seattle Focus. In today's program, we have a special guest from Martha@Home Co., one of the leading companies in Seattle. Recently, the company has been involved in a series of news, from a fire incident one month ago to a nationwide recall of their most popular product Martha@Home M-10 coffeemaker. There have been quite some concerns in the public about the overall product safety of the company. And today's guest will have more to say about the company. For the confidentiality reason, his voice has been processed. Good evening!

Guest: Good evening!

Host: So how many years have you been working for Marth@Home Co.?

Guest: About four years.

Host: So you know the company quite well.

Guest: Yes. I have been working in two departments in Seattle. At least, I could say that I know the operation of the Seattle headquarter pretty well. 
Host: So why are you here tonight?

Guest: As you just said, lately there have been rumors and public concerns about the product safety of Martha@Home Co. As a person close to the situation, I just want to inform the public about some conducts of Martha@Home Co. For example, unlike most other companies, Martha@Home Co. does not prohibit procurement personnel from receiving personal gifts from other companies. Of course, cash and luxurious gifts are prohibited by the company's ethic codes. But in practice, nobody in the procurement department really cares about these codes, especially those guys at the top. The procurement manager used to receive a lot of free meals from our suppliers in the best restaurants of Seattle and sometime even secret "bonuses" from some suppliers according to how much he decides to purchase ...

\section{Computer-Participant interface}

An anonymous employee went onto a local television and revealed that it is a common practice for the procurement department of Martha@Home Co. to receive personal gifts from its suppliers. For example, the procurement manager used to receive "volume-based incentive bonus" from the current supplier of the defective inflammable plastic component of the Martha@Home M-10 model. You decide

A. To appear in the same local television, apologizing for the wrongdoing of the company's procurement department and promising to tighten the implementation of the company's ethic codes.

B. To appear in a major national television, revealing all the information that you have from the household fire incident to the recent appearance of the anonymous employee on the local television and explaining the company's ethic codes and endeavors to protect the best interests of all stakeholders in the current crisis situation. C. To find out the anonymous employee, and appear in a major local television, explaining that the top management was not aware of every detail of the company's procurement practice and admonishing the public not to believe everything that the anonymous employee has said.

\section{Scenario 8}

\section{Information introduced in audio}

(Telephone is ringing)

Jack Smith: Good morning! This is Jack Smith, the head of the legal department. I heard a whisper from my old college buddies, who are now working in the justice department that the justice department is thinking of setting up a special team to investigate our company over the deal we had with Priority Inc. one year ago. Man, I can tell you, it will bring us into deep trouble if the decision is finally made . . . 
But, as my fellows told me, maybe we could kind of "lobby" with the justice department and convince them it is not necessary to have the investigation ...

\section{Computer-Participant interface}

8. You heard a whisper that the city's justice department is thinking of setting up a special investigation team to investigate the suspected misconducts of Martha@Home procurement manager. The head of the company's legal department told you that he has some personal networks with the justice department and that the company could "lobby" against having such an investigation team. You would

A. Accept his suggestion, since no matter what results the investigation brings up, the investigation itself would further jeopardize the company's reputation.

B. Not accept his suggestion, since no matter what results the "lobbying" brings up, the lobbying itself, if known to the public, would further jeopardize the company's reputation.

\section{Scenario 9}

\section{Information introduced in texts/ Computer-Participant interface}

The stock price of Martha@Home Co. has dropped 8.4\% since the nationwide recall of the M10 coffeemakers. Moreover, two biggest supermarket chains in the US have just decided to cancel their million-dollar orders of small electronic kitchenware (e.g. toasters, water boilers) with the company, due to a concern of product safety. You think the company should

A. Lay off employees to signal the financial market that Martha@Home Co. would put the company's profits and investors' interest top priority at the moment, and offer discounts to the two supermarket chains to maintain their contracts.

B. Lay off employees to signal the financial market that Martha@Home Co. would put the company's profits and investors' interest top priority at the moment, and invite quality inspectors and procurement managers of the two supermarket chains to test product safety in order to relieve their concerns.

C. Not lay off employees, since it is more important to maintain morale of the company than to please the financial market at the moment, and invite quality inspectors and procurement managers of the two supermarket chains to test product safety in order to relieve their concerns.

\section{Scenario 10}

\section{Information introduced in audio}

Jack Smith: Good morning! This is Jack Smith from the legal department. We received some news from the city's police department yesterday afternoon. And their investigation results of that fire incident about six weeks ago suggest the fire was actually caused by a very old cable connected to a fridge in the kitchen. It was just a 
coincidence that an M10 coffeemaker was working at that moment, which led the police thought it was the coffee machine that caused the fire. But, you know what, just several hours before we got the news from the police, Tom Robinson, the procurement manager, admitted that he received a 50,000-dollar check from a sale representative of Priority Inc. after he decided to give them the three-year contract! Tom said that he was aware that the quality of the inflammable plastic component was slightly lower than our safety standard. What a dramatic result ...

\section{Computer-Participant interface}

10. The city's police department just found out that the house fire was caught by an old electricity cable connected to a fridge in the kitchen that was next to a working Martha@Home M10 coffeemaker. But, the procurement manager admitted receiving a 50,000-dollar bonus from the supplier of the defective plastic component for signing a three-year purchasing contract, even though the quality of the component is slightly below Martha@Home's safety standard. You decide

A. To make an announcement in the Seattle Times about the results of the police investigation, inform the public of the misconducts of the procurement manager, and communicate "the happy ending" of the story to the employees.

B. To go to a major national television, "publicizing" how Martha@Home Co. navigated the crisis step by step and what the company has learned from the experience, and send emails to all stakeholders - customers, business partners, and stockholders, informing them of "the happy ending" of the story.

C. To send emails to employees, affected customers, retailers, distributors, and stockholders, informing them of the results of the police investigation and the misconducts of the procurement manager but not to go to any mass medium.

\section{Answer Keys}

CCACACBBCB 



\section{Summary}

Teams have become a popular format for nowadays organizations to organize and accomplish increasingly complex tasks. Team researchers commonly use an InputProcess-Output (IPO) framework, which explains team outputs (e.g. team performance, team cohesion) from team inputs (e.g. team members' skills, demographics, and personality traits) and intermediary team processes (e.g. team communication, conflict). In this dissertation, we examine two critical temporal conditions underlying the IPO framework, develop new methods to study temporal dynamics, and suggest avenues for advancing team research.

In Chapter 1, we identify these two conditions, that is, team inputs must be stable over time and across situations, and teams must differ in their interactions over time (i.e. team processes) and analyze how they are studied in the extant organizational behavior literature. Our analysis reveals methodological drawbacks in earlier research, particularly the use of the test-retest reliability coefficient and of the inter-subject longitudinal approach as a means to examine intra-subject temporal dynamics. We suggest that methodological improvement is needed to advance research based on the IPO framework and to develop pertinent team theories.

In Chapter 2, we examine the first condition by testing the intertemporal stability of time urgency, polychronicity, and time perspective via the alpha-betagamma-change typology and latent growth models. We also test the intertemporal stability of temporal depth via repeated-measures ANOVA. With the data from a three-wave survey with 63 faculty members and students from the School of Business and Economics of Maastricht University over six months (189 data points), we find that most temporal characteristics, including eating behavior, general hurry, and task-related hurry of time urgency, polychronicity, and past-positive, pastnegative, and present-hedonistic of time perspective, vary in construct validity over time. Competitiveness of time urgency varies in construct calibration over time. Future orientation of time perspective and temporal depth are the most stable temporal characteristics, both demonstrating stable construct score over time. The low intertemporal stability of the four temporal characteristics highlights the necessity for team researchers to examine the intertemporal stability of presumably stable deep-level team inputs (e.g. Big Five personality traits, values) via the alpha-betagamma-change typology and latent growth modeling rather than the test-retest reliability coefficient, in order to better theorize and test the mediating effect of deeplevel team inputs (i.e. psychological attributes) on the relationship between surfacelevel team inputs (i.e. demographic features) and team outputs. 
In Chapter 3, we examine the first condition by testing the intersituational stability of time urgency, polychronicity, time perspective, and temporal depth via a quasiexperiment. With the data from 101 students from the School of Business and Economics of Maastricht University over a one-month period (202 data points), we find that some temporal characteristics change across situations. The quasi-experiment consisted of a control condition, in which a group of participants were exposed in two weak situations and an experimental condition, in which a group of participants were first exposed in a weak situation and then a strong situation with a computer simulation of corporate crisis management. The simulation was designed to increase the participants' time urgency and present time perspective and to decrease their polychronicity and temporal depth. We find that task-related hurry of time urgency, hedonistic-present and future orientation of time perspective are subject to situational influences, demonstrating equivalent factor structure over time within each condition but variant factor structures between the two conditions. In comparison, eating behavior and general hurry and temporal depth are rather stable across situations, demonstrating equivalent attribute scores over time and between the two conditions. Furthermore, we are unable to conclude upon the intersituational stability of competitiveness of time urgency, polychronicity, and past-positive, past-negative, and present-fatalistic of time perspective, because their factor structures vary over time in at least one of the two conditions.

In Chapter 4, we discuss the logical connections between group-level temporal dynamics and individual-level temporal dynamics. We suggest that the occurrence of group-level attribute change over time implies the occurrence of individual-level change, but not vice versa, and that attribute stability over time at the individual level implies stability at the group level, but not vice versa. Based on this discussion, we note that the inter-subject longitudinal approach that is conventionally used in connection with the IPO-framework- does not answer the research questions regarding the impact of team processes on team outputs. Whereas the purpose of the theoretical inquiry is to examine associations between inter-team differences in team input levels, team process dynamics, and team output levels, the inter-subject approach assumes homogeneous or invariant process dynamic patterns of individual teams (assuming that the estimated group-level pattern applies to all individual teams). Starting from a reversed logic, assuming heterogeneity and using an individuals-to-group aggregation method, we develop an intra-subject longitudinal approach that allows to examine the second temporal condition and to explore the nomological networks of team dynamics.

In Chapter 5, we apply the intra-subject approach and inter-subject approach to a longitudinal team study. With the data from 42 project teams of business graduate students from the School of Business and Economics of Maastricht University over two months (126 team-level data points), we find that the temporal dynamics of team conflict relates differently to team satisfaction than the level of team conflict at the beginning, middle, and end of team projects. Results from the 
intra-subject and inter-subject approach suggest that teams with decreased task and process conflict during the second half of the project time, regardless of their developmental patterns during the first half, report higher team satisfaction than the other teams. Moreover, results from the intra-subject approach show that teams with continuously increased relationship conflict throughout team projects report higher team satisfaction than the other teams, whereas results from the inter-subject approach suggest that the high- and low-satisfaction teams differ only in the developmental pattern of team task and process conflict in the first half of the project time. We did not find any relationship of team conflict over time with team performance in either approach but a link with team task conflict level at the midpoint and the end and team process conflict level at the end. A remarkable finding is that teams differ widely in their conflict dynamic patterns over time. Out of the 42 teams, only $5 \%, 10 \%$ and $2 \%$ demonstrate the same pattern as the sample-level pattern for task, relationship, and process conflict respectively.

In Chapter 6, we derive theoretical implications of the dissertation for future team research. We propose to test the stability of team psychological inputs over time and across situations, and to study the temporal dynamics in its own right. Furthermore, we suggest that the intra-subject longitudinal approach and intersubject longitudinal approach address different theoretical aspects of a researched phenomenon, that is, the temporal dynamics regardless of its level and the level at particular time moments. Finally, we argue for the application of the intra-subject approach to researching all aspects of the IPO framework - that is, all inputs, processes and outputs - as well as in the study of individual persons' attitudes and behaviors. 



\section{Samenvatting (Summary in Dutch)}

Teams zijn uitgegroeid tot een populair medium in huidige organisaties om steeds meer complexere taken te managen. Team onderzoekers gebruiken vaak een inputproces-output (IPO) model, welke 'team output' (bijv. teamprestatie, teamcohesie) via 'team inputs' (bijv. vaardigheden van teamleden, demografie, en persoonlijkheidskenmerken) en tussenliggende teamprocessen verklaart (bijv. teamcommunicatie, conflicthantering). In dit proefschrift onderzoeken we twee kritische temporele voorwaarden die ten grondslag liggen aan het IPO model, ontwikkelen we nieuwe methoden om de temporele dynamiek te bestuderen, en stellen we nieuwe directies voor om team onderzoek te bevorderen.

In hoofdstuk 1, identificeren we deze twee voorwaarden, dat wil zeggen, team inputs moeten stabiel zijn over tijd en over situaties en teams moeten verschillen in hun interacties in de tijd (dwz. teamprocessen). Ook analyseren we hoe ze worden bestudeerd in de bestaande Organisatie Gedrag literatuur. Onze analyse toont methodologische bezwaren aan in eerder onderzoek, in het bijzonder in het gebruik van de test-hertest betrouwbaarheid coëfficiënt en in de inter-subject longitudinale benadering als een middel om intra-subject temporele dynamiek te onderzoeken. Wij suggereren dat methodologische verbetering nodig is wat betreft onderzoek naar het IPO model en de ontwikkeling van pertinente team theorieën.

In hoofdstuk 2 onderzoeken we de eerste voorwaarde door het testen van de inter-temporele stabiliteit van 'tijdurgentie', 'polychroniciteit' en het tijdsperspectief op basis van de 'alfa-bèta-gamma-verandering' typologie en latente groeimodellen. We testen ook de inter-temporele stabiliteit van temporele diepte via 'repeated-measures' ANOVA. Met de gegevens uit een drie-wave enquête met 63 docenten en studenten van de Maastricht University School of Business and Economics, over een tijdsbestek van 6 maanden (189 datapunten), vinden we dat de meeste temporele kenmerken, met inbegrip van eetgedrag, algemene haast, en taakgerelateerde haast in tijdurgentie, polychroniciteit, en 'past-positive', 'past-negative', en 'present-hedonistic' in tijdsperspectief, variëren in constructvaliditeit over tijd. Concurrentievermogen van tijdurgentie varieert in construct calibratie over tijd. Toekomstoriëntatie in het tijdsperspectief en temporele diepte zijn de meest stabiele temporele kenmerken, beiden demonstreren stabiele constructscores over tijd. De lage inter-temporele stabiliteit van de vier temporele kenmerken benadrukt de noodzaak om de inter-temporele stabiliteit van de vermoedelijke stabiele 'deeplevel team inputs' (bijv. Big Five persoonlijkheidskenmerken en waarden) via de 'alfa-bèta-gamma-verandering' typologie en latente groeimodellering te toetsen in 
plaats van met de test-hertest betrouwbaarheid coëfficiënt. Dit is nodig om het bemiddelende effect van 'deep-level team inputs (dwz. psychologische kenmerken) op de relatie tussen 'oppervlakte niveau team inputs' (dwz. demografische kenmerken) en 'team-output' beter te kunnen theoretiseren en testen.

In hoofdstuk 3 onderzoeken we de eerste voorwaarde door het testen van deintersituatioelel stabiliteit van 'tijdurgentie', 'polychroniciteit', het 'tijdsperspectief', en temporele diepte via een quasi-experiment. Met de gegevens van 101 studenten van de Maastricht University School of Business and Economics over een periode van één maand (202 datapunten), vinden we dat sommige temporele eigenschappen veranderen over de situaties. Het quasi-experiment bestond uit een controle conditie, waarin een groep van deelnemers werd blootgesteld aan twee zwakke situaties en een experimentele conditie, waarbij een groep deelnemers eerst werd blootgesteld aan een zwakke situatie bevindt en vervolgens een sterke situatie met een computersimulatie over corporate crisisbeheersing. De simulatie was bedoeld om de 'tijdurgentie' en huidig 'tijdsperspectief' van deelnemers te vergroten en hun 'polychroniciteit' en 'temporele diepte' te verlagen. We vinden dat 'taakgerelateerde haast van tijdurgentie', 'hedonistic-present' en 'toekomstoriëntatie van tijdsperspectief' onderhevig zijn aan situationele invloeden. Hieruit blijkt een gelijkwaardige factorstructuur over de loop van de tijd binnen elke conditie, maar een variant factorstructuren tussen de twee condities. Ter vergelijking, 'eetgedrag' en 'algemene haast' en 'temporele diepte' zijn vrij stabiel over de situaties, waaruit blijkt dat de attributiescores over tijd en tussen de twee condities gelijkwaardig zijn. Bovendien zijn wij niet in staat iets te concluderen over de inter-situationele stabiliteit van het 'concurrentievermogen over tijdurgentie', 'polychroniciteit', en 'past-positive' and 'past-negative', en de 'presnet-fatalistic van tijdsperspectief', omdat hun factorstructuren over tijd in ten minste een van de twee condities variëren.

In hoofdstuk 4 bespreken we de logische verbanden tussen groepsniveau temporele dynamiek en individueel temporele dynamiek. Wij suggereren dat het voorkomen van verandering van kenmerken op groepsniveau over tijd een verandering in het voorkomen op individueel niveau impliceert, maar niet vice versa. Tevens suggeren we dat stabiliteit in kenmerken over tijd individueel niveau stabiliteit op groepsniveau impliceert, maar niet andersom. Op basis van deze discussie, stellen we vast dat de inter-subject longitudinale aanpak die normaal gebruikt wordt in relatie tot het IPO model, niet de onderzoeksvragen beantwoordt over de gevolgen van teamprocessen op 'team outputs'. Terwijl het doel van het theoretisch onderzoek is om associaties tussen inter-team verschillen in team input niveaus, teamproces dynamiek en teamprestaties te onderzoeken, veronderstelt de inter-subject benadering homogene of invariante dynamische procespatronen van individuele teams (gegeven dat het geschatte groepsniveau patroon geldt voor alle individuele teams). Vertrekkend vanuit een omgekeerde logica en uitgaande van heterogeniteit en het gebruik van een individu-naar-groep aggregatie, ontwikkelen we een longitudinaal 
intra-subject benadering die het toelaat om de tweede temporele conditie te onderzoeken, evenals de nomologische netwerken van teamdynamiek verkent.

In hoofdstuk 5, passen we de intra- en inter-subject benadering toe op een longitudinale teamstudie. Met de gegevens uit 42 projectteams bestaande uit afgestudeerde bedrijfskunde studenten van de Maastricht University School of Business and Economics en data verzameld over een periode van twee maanden (126 teamlevel datapunten), vinden we dat de temporele dynamiek van teamconflicten zich verschillend onderhoudt ten opzichte van de tevredenheid van het team dan ten opzichte van het niveau van teamconflict in het begin, midden en einde van de projecten. Resultaten van de intra- en inter-subject benadering suggereert dat teams met verminderd taak en proces conflict in de tweede helft van de totale projecttijd hogere teamtevredenheid rapporteren dan andere teams. Ongeacht hun ontwikkelingspatronen in de eerste helft. Bovendien, tonen de resultaten van de intra-subject benadering dat teams met voortdurend gestegen relatieconflict, hogere teamsatisfactie rapporteren dan de andere teams, terwijl de resultaten van de inter-subject benadering erop wijzen dat de hoge-en lage-tevredenheid teams alleen verschillen in de ontwikkelingspatronen van taak en proces conflict in de eerste helft van de projecttijd. We vinden geen relatie van teamconflict met de teamprestatie, over tijd, ongeacht de benadering, maar een wel een link met taakconflict op het midden en het einde en procesconflict aan het eind. Een opmerkelijke bevinding is dat de teams sterk verschillen in hun dynamische conflictpatronen over tijd. Van de 42 teams, rapporten slechts $5 \%, 10 \%$ en $2 \%$ hetzelfde patroon als in de steekproef voor respectievelijk taak, relatie en procesconflict.

In Hoofdstuk 6, bespreken we de theoretische implicaties van het proefschrift voor toekomstig teamonderzoek. Wij stellen vast dat het belangrijk is om de stabiliteit van team psychologische inputs over tijd en situaties te toetsen en de temporele dynamiek in zijn eigen recht te bestuderen. Verder suggereren we dat de longitudinale intra- en inter-subject benadering andere theoretische aspecten belicht in het onderzochte fenomeen. Dat wil zeggen, de temporele dynamiek, ongeacht het niveau en het niveau op bepaalde tijdstippen. Ten slotte pleiten we voor de toepassing van de intra-subject benadering in al het IPO model onderzoek (input, process, output) alsook in de studie naar individueel gedrag en attitudes. 



\section{About the Author}

Jia Li was born in Hechi, China, on July $30^{\text {th }}$, 1982. She graduated from Guilin High School in 2000. From 2000 to 2004, she studied Business Administration in Zhejiang Gongshang University and graduated with a degree of Bachelor of Science (with honor). From 2004 to 2006, she studied Business Research in Maastricht University, the Netherlands, and obtained a degree of Master of Philosophy.

In August 2006, she started to work on a PhD project "The Nature and Role of Time Perspective in Organizations and Teams" in the Department of Organization and Strategy at Maastricht University. The project later evolved into the current dissertation entitled "Time in Teams: Methodological Issues in the Study of Temporal Dynamics". From January 2010 to present, she has worked as a lecturer in the Department of Organization and Strategy at Maastricht University. 